\author{
Universidade de São Paulo \\ Instituto de Fŕsica
}

\title{
Misturas Homogêneas de Átomos \\ Bosônicos com Acoplamento de Josephson \\ a Temperatura Finita
}

\section{Marcelo Oliveira da Costa Pires}

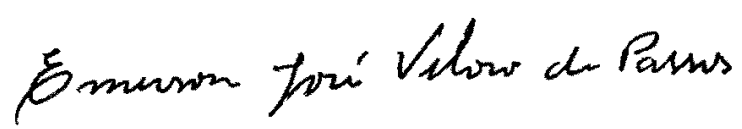

Orientador : Prof. Dr. Emerson José Veloso de Passos

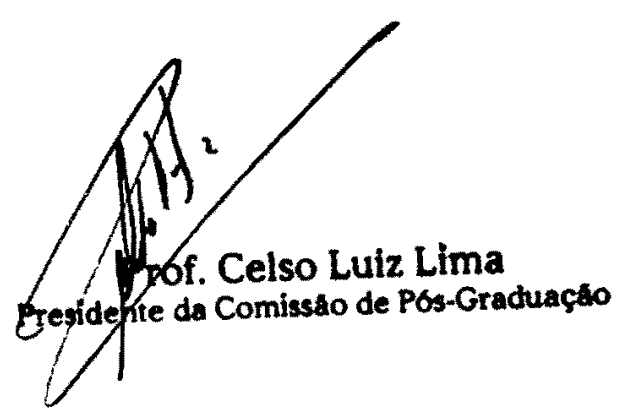

Comissão Examinadora

Prof. Dr. Emerson José Veloso de Passos (Orientador, IFUSP)

Prof. Dr. Antonio Fernando Ribeiro de Toledo Piza (IFUSP)

Prof. Dr. Arnaldo Gammal (IFUSP)

Prof. Dr. Lauro Tomio (IFT/UNESP)

Prof. Dr. Frederico Firmo de Souza Cruz (UFSC)

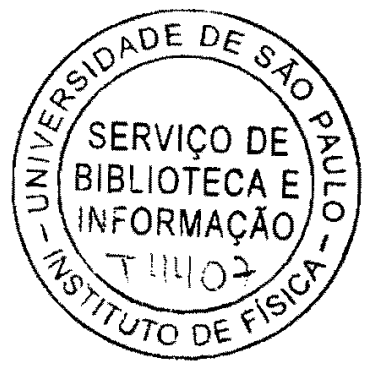

São Paulo

2005
Tese de doutorado apresentada no Instituto de Física para a obtenção do título de Doutor em Ciências.

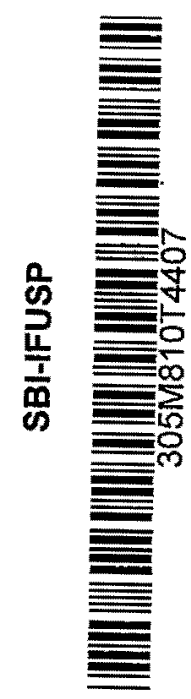




FICHA CATALOGRÁFICA
Preparada pelo Serviço de Biblioteca e Informação
do Instituto de Física da Universidade de São Paulo
\begin{tabular}{|l} 
Pires, Marcelo Oliveira da Costa \\
Misturas Homogêneas de Átomos Basônicos com \\
Acoplamento de Josephson a Temperatura \\
Finita. São Paulo, 2005. \\
Tese (Doutoramento) - Universidade de São Paulo \\
Instituto de Física - Depto. de Física Matemática \\
Orientador: Prof. Dr. Emerson José Veloso de Passos \\
Área de Concentração: Física \\
Unitermos: \\
1. Física Atômica; \\
2. Condensado de Bose - Einstein - Misturas Coerentes; \\
3. Condensado de Bose Einstein - Temperatura \\
Finita. \\
USP/IF/SBI-078/2005
\end{tabular}


"Nada estimo mais, entre todas as coisas que não estão em meu poder, do que contrair uma aliança de amizade com seres que amem sinceramente a verdade." Espinosa 
Dedico aos meus pais e a Júlia. 


\section{Resumo}

Usando o princípio variacional de máxima entropia, deduzimos as equações de GrossPitaevskii e de Hartree-Fock-Bogoliubov que descrevem, respectivamente, os estados de equilíbrio e as excitações coletivas de uma mistura homogênea de átomos bosônicos em dois estados hiperfinos diferentes, à temperatura finita e na presença de um termo de acoplamento de Josephson interno. Para corrigir o problema da ausência de um ramo sem lacuna do espectro de energia de excitação na teoria de Hartree-Fock-Bogoliubov, mostramos como estender a aproximação de Popov para o caso de misturas de condensados. Para temperaturas abaixo da temperatura de transição do condensado, calculamos, como função da temperatura, o número de partículas em cada condensado, o espectro das excitações coletivas, a lacuna e a velocidade do som. Examinamos como a bi-estabilidade do sistema muda com a temperatura. Quando aquecemos a mistura, dependendo dos valores dos parâmetros do sistema, verificamos que a bi-estabilidade desaparece para uma temperatura menor que a temperatura de transição, ou pela instabilidade de um dos estados de equilíbrio, ou pela degenerescência dos dois estados de equilíbrio estável. 


\begin{abstract}
We use the principle of maximum entropy to derive the Gross-Pitaevskii and the HartreeFock-Bogoliubov equations which describe the equilibrium states and the collective excitations of a binary homogeneous mixture of bosonic atoms in two different hyperfine states, in the presence of an internal Josephson coupling, at a finite temperature. To correct the absence of a gapless excitation branch in the Hartree-Fock-Bogolibov theory, we show how to extend the Popov approximation to the case of condensate mixtures. We calculate, as function of the temperature, physical quantities such as the fraction of atoms in the condensates, the spectra of collective excitations, the gap and the speed of sound. We investigate how the bistable structure found at null temperature changes when we increase the temperature, starting at $T=0$. When one heat the mixture, depending on the values of the system's parameters, we note that the bi-stability disappears at a temperature smaller than the transition temperature, either by the instability of one of the equilibrium states or by the degeneracy of the two stable equilibrium states.
\end{abstract}




\section{Agradecimentos}

- Agradeço, primeiramente, ao Prof. Emerson pela orientação durante a realização desse trabalho.

- Agradeço ao Dr. Paolo Tommasini pela sugestão do tema da tese.

- Agradeço às pessoas que se dedicam ao estudo de condensados no departamento de Física-Matemática, entre elas, Prof. Piza, Fabio Paolini, Dimas Romano, Leonardo Sioufi, Lin e Andrea Nemes.

- Agradeço a todas as pessoas que conviveram comigo no Departamento de FísicaMatemática, em especial as secretárias: Amélia, Simone e Beth; a turma da computação do departamento: João e Sybele, e aos amigos Akira, Alessandro, Alex, Carlos, Edivaldo, Everton, José Cleriston, Kleber, Mario, Michele, Milton, Prof. Molina, Rafael, Ronaldo e Tiago.

- Agradeço aos colegas pessoais, Adriana, Cida, Claudia, Claudio, Flavia, Julia, Marcelo Bussoti, Patrícia e Simone Kugeratski.

- Agradeço a meu pai Martinho, a minha mãe Nadete, a meu irmão Michel e à Carol.

- Finalmente, agradeço à FAPESP pelo apoio financeiro que possibilitou a realização desse trabalho. 


\section{Conteúdo}

1 Introdução $\quad 4$

1.1 Um pouco de história . . . . . . . . . . . . . . . . . . 4

1.2 As experiências . . . . . . . . . . . . . . . 6

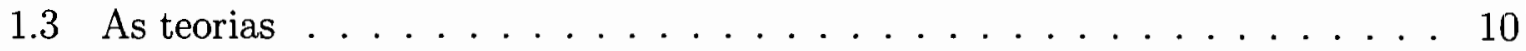

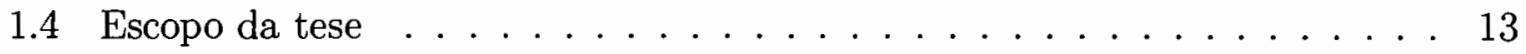

2 A teoria e suas aproximações $\quad 14$

2.1 O hamiltoniano do sistema . . . . . . . . . . . . . . . . 14

2.2 Princípio de entropia máxima . . . . . . . . . . . . . . 17

2.3 Determinação do hamiltoniano grão-canônico efetivo . . . . . . . . . . 18

2.4 Equações de Hartree-Fock-Bogoliubov dependente da temperatura . . . . . 21

2.5 Equações de Gross-Pitaevskii dependente da temperatura . . . . . . . . . . . 24

2.6 Invariância de calibre do sistema . . . . . . . . . . . . . . . . 26

2.6.1 Simplificações nas equações de Gross-Pitaevskii . . . . . . . . . . 27

2.6.2 As energias das excitações coletivas . . . . . . . . . . . . . 31

2.7 Aproximação de Popov . . . . . . . . . . . . . . . . . . . 32

2.7.1 A equação de Gross-Pitaevskii na aproximação de Popov . . . . . . 34

2.7.2 A lacuna e a velocidade do som na aproximação de Popov . . . . . 35

3 Apresentação e comentários dos resultados numéricos 38

3.1 Método da solução das equações de Gross-Pitaevskii e de Hartree-Fock-

Bogoliubov-Popov . . . . . . . . . . . . . . . . . . 38

3.2 Um caso "realista" . . . . . . . . . . . . . . . . . . . . . . . 42

3.3 Um caso na região de bi-estabilidade com uma dessintonia efetiva diferente de zero . . . . . . . . . . . . . . . . . . 47 
3.4 Um caso na região de bi-estabilidade com uma dessintonia nula . . . . . 53

4 Conclusão $\quad 57$

$\begin{array}{ll}\text { A O teorema de Wick em mecânica estatística } & 61\end{array}$

B Determinação das temperaturas de transição $\quad 65$

B.1 Para um sistema ideal de uma componente . . . . . . . . . . . 65

B.2 Para um sistema ideal de duas componentes com acoplamento Josephson . 66

B.3 Para um sistema interagente de duas componentes com acoplamento Josephson .............................. 68 


\section{Capítulo 1}

\section{Introdução}

"O conhecimento do efeito nada mais é que adquirir um mais perfeito conhecimento da causa."

Espinosa

\subsection{Um pouco de história}

A obtenção do condensado de Bose-Einstein em gases diluídos de átomos alcalinos em 1995 [1, 2, 3] abriu um excitante caminho para a mecânica quântica e, em especial, serviu de um grande laboratório para as teorias de muitos corpos. Podemos dizer, sem exageros, que uma nova era iniciou-se no estudo da matéria condensada. Ciente disso, a comunidade científica laureou com o prêmio Nobel os físicos Carl Wieman, Eric Cornell e Wolfgang Ketterle [4,5] apenas seis anos após terem conseguido produzi-lo em laboratório.

Contudo, a história dos condensados não começa em 1995 com a descoberta experimental, ela origina-se nos primórdios da mecânica quântica quando, em 1924, Bose desenvolve uma nova estatística, mais tarde denominada de "estatística de Bose-Einstein", para deduzir a lei de Planck para a radiação de corpo negro [6]. Por problemas de publicação Bose pediu recomendação a Einstein que reconheceu o valor do trabalho. Em 1925, Einstein estendeu a aplicação da nova estatística de fótons para qualquer objeto massivo [7] e como resultado dessa extensão observou uma condensação de partículas no estado fundamental motivada unicamente pela estatística, sem a presença das interações. Era a primeira vez que alguém falava de transição de fase para o gás ideal.

Na época que Einstein fez essa descoberta, muitos opunham-se à realidade desse 
fenômeno, acreditando ser um caso patológico do gás ideal e que desapareceria tão logo levá-se em conta a interação entre as partículas. Entretanto, passados 14 anos, London [8] reconsiderou a idéia notando que, para um gás ideal com a massa e a densidade do ${ }^{4} \mathrm{He}$, a temperatura na qual ocorre o fenômeno de condensação, $T_{0}=3.13 \mathrm{~K}$, é muito próxima da temperatura de transição da fase superfluida do gás, $T_{\lambda}=2.19 \mathrm{~K}$. Assim, associou o fenômeno da superfluidez como uma manifestação da presença do condensado no fluido.

As idéias de London inspiraram Tisza [9] a propor um modelo fenomenológico de dois fluidos, um fluido de partículas condensadas e outro fluido de partículas ordinárias. $\mathrm{O}$ fluido das condensadas comportar-se-ia como um superfluido apresentando propriedades de viscosidade nula e as partículas fora do condensado pertenceriam ao fluido normal.

Landau, por outro lado, opôs-se às idéias de Tisza de associar a componente condensada como sendo a componente superfluida e propôs uma teoria hidrodinâmica de dois fluidos, $[10,11,12]$, na qual considera a componente superfluida como a parte do líqüido que permanece no estado fundamental com entropia nula e a componente normal como as excitações de quasi-partículas do sistema. Dessa forma, as propriedades de transporte e a termodinâmica de baixa temperatura do ${ }^{4} \mathrm{He}$ superfluido são conseqüências da excitação coletiva do superfluido ser proporcional ao momento dessa excitação no limite de baixo momento [13].

A teoria de Landau é fenomenológica pois tanto as propriedades de superfluidez quanto a natureza do espectro de excitação são postulados de modo intuitivo. Faltava uma descrição microscópica dos fenômenos dada por Bogoliubov, [14], no caso em que consideramos o fluido como um gás, e depois por Feynman e Cohen, $[15,16]$, para o caso em que consideramos o fluido como líquido.

Bogoliubov considerou um gás diluído de átomos que obedecem a estatística de BoseEinstein e interage uns com os outros através de uma interação binária fracamente repulsiva. Ele assumiu que em tal sistema, como no caso do gás ideal, ocorre o fenômeno de condensação, e então, usando uma série de aproximações controladas que veremos adiante, ele foi capaz de mostrar que o espectro de energia para grandes momentos corresponde aproximadamente a uma excitação de átomos não interagentes do condensado e para momento pequeno o espectro é fonônico como postulado por Landau.

A teoria de Bogoliubov não é válida para o ${ }^{4} \mathrm{He}$ superfluido. Apesar da teoria descrever propriedades de superfluidez, a teoria falha na descrição do líquido pois os átomos estão 
tão próximos um dos outros que ficam sensíveis a forma da interação de van der Waals.

Feynman e Cohen, por sua vez, desenvolveram uma teoria macroscópica para o ${ }^{4} \mathrm{He}$ líquido com base no método variacional. Entre outras coisas, esses trabalhos mostraram que o espectro de excitação tem um comportamento de fónons em baixos momentos e um de rótons para momentos maiores [16]. Não entramos em detalhe dessa teoria pois, como veremos, estamos interessados num sistema diluído.

\subsection{As experiências}

Desde a previsão teórica dos condensados por Bose e Einstein até a obtenção dos condensados em átomos bosônicos alcalinos de ${ }^{87} \mathrm{Rb}$ [1], ${ }^{23} \mathrm{Na}$ [2] e ${ }^{7} \mathrm{Li}$ [3], o único fenômeno onde se observava a presença do condensado atômico de Bose-Einstein era a transição do ${ }^{4} \mathrm{He}$ líquido para a fase superfluida [13]. Levaram-se 70 anos da predição de Einstein até que uma combinação de técnicas de resfriamento por evaporação e por laser numa armadilha magneto-ótica conduziu ao sucesso a busca da condensação de gases de átomos alcalinos.

Nos átomos de ${ }^{87} \mathrm{Rb},{ }^{23} \mathrm{Na} \mathrm{e}^{7} \mathrm{Li}$, todas as camadas eletrônicas são preenchidas exceto uma última camada $s$ que contribue com spin, $S=1 / 2$, para o momento angular total do átomo. Por esses átomos serem neutros, o número de prótons tem que ser ímpar, e como o núcleo desses átomos tem peso atômico ímpar, o número de nêutrons é par. Como conseqüência, vemos que o momento angular total, $F=I \pm 1 / 2$, (nuclear mais eletrônico) desses elementos é inteiro e que, portanto, o sistema é simétrico pela troca de duas partículas e obedece a estatística de Bose-Einstein o que torna possível sua condensação.

Confina-se esses elementos através do aprisionamento magneto-óptico. A técnica consiste na associação de um campo magnético não homogêneo com um mínimo no centro da armadilha e da utilização de feixes de laseres polarizados apontados para o centro [17]. A associação dessas técnicas de confinamento e resfriamento faz diminuir a temperatura do sistema confinado num potencial harmônico anisotrópico, tornando possível obter uma densidade no centro do potencial em torno de $10^{11} \mathrm{~cm}^{-3}$ a $10^{15} \mathrm{~cm}^{-3}$ e uma temperatura extremamente baixa, de centenas de nano-Kelvin a dezenas de micro-Kelvin. Por ser um gás diluído em temperaturas baixas, a interação entre duas partículas é dada por um po- 
tencial de contato parametrizado pelo comprimento de espalhamento de onda $s$ da ordem de nanometros. Essas condições de temperatura, densidade e interação entre partículas no sistema permitiram os experimentais obter de dezenas de milhares a milhões de partículas condensadas num regime meta-estável de dezenas de segundos [18].

Para termos uma idéia qualitativa de quão são baixas as energias envolvidas no sistema, comparamos, na tabela 1.1, as ordens de magnitudes típicas das várias energias relevantes de um conjunto de $10^{6}$ átomos ultrafrios de ${ }^{87} R b$ numa armadilha magnética típica [18],

Tabela 1.1: Energias para um conjunto de $10^{6}$ átomos de ${ }^{87} R b$ numa típica armadilha magnética.

\begin{tabular}{|c|c|}
\hline \hline & Unidades de freqüência \\
\hline Energia da transição $n s \rightarrow n p$ & $\approx 4 \times 10^{14} \mathrm{~Hz}$ \\
Energia de interação entre dois átomos $\hbar^{2} / 2 m a_{s}^{2}$ & $\approx 2 \times 10^{6} \mathrm{~Hz}$ \\
Temperatura de transição $k_{B} T_{0}$ & $\approx 10 \mathrm{kHz}$ \\
Energia de campo médio $\lambda \rho$ & $\approx 2 \mathrm{kHz}$ \\
Energia de ponto zero do poço harmônico $\hbar \omega_{0}$ & $\approx 100 \mathrm{~Hz}$ \\
\hline
\end{tabular}

A primeira quantidade da tabela 1.1 é a energia da transição entre estados eletrônicos num átomo isolado de ${ }^{87} R b$ e não depende das condições de confinamento. Observa-se que esse valor é $10^{10}$ vezes maior que a energia correspondente a temperatura de transição desses átomos nessas condições de densidade. A energia correspondente a temperatura de transição é maior que a energia de campo médio do sistema e muito maior que a energia de ponto zero da armadilha.

Em condições usuais das experiências, o comprimento de espalhamento de onda $s$, $a_{s} \approx 5 \mathrm{~nm}$, é duas ordens de grandeza menor que a distância média entre as partícula, $\approx 450 \mathrm{~nm}$ para uma densidade de $10^{13} \mathrm{~cm}^{-3}$. Isso permite considerar o sistema como sendo diluído e caracterizar o estado do condensado como produto de estados de partículas independentes.

Após a descoberta dos condensados em átomos alcalinos diluídos, vários grupos experimentais mediram as energias das excitações coletivas do sistema. A primeira técnica proposta e realizada foi excitar os estados coletivos através da modificação do potencial de confinamento [19, 20]. Uma outra técnica usa a espectroscopia de Bragg de dois fótons sugerida em [21] para medir o fator de estrutura do condensado num regime de 
fónons [22], verificando que essa quantidade está de acordo com as predições do gás homogêneo de Bose usando uma aproximação de densidade local. Usando a mesma técnica foi possível observar o espectro de excitação no centro da armadilha, o que permitiu extrapolar medidas do espectro de excitação de um condensado confinado para um condensado homogêneo. Observou-se um comportamento fonônico do espectro de excitação [23] e o seu respectivo modo [24] estão de acordo com as previsões da teoria de Bogoliubov para o caso homogêneo.

O próximo desafio experimental foi a observação de misturas de condensados. Num dos primeiros trabalhos [25] conseguiu-se condensar uma mistura de átomos em diferentes estados hiperfinos. A técnica consiste em produzir um condensado de ${ }^{87} R b$ no estado hiperfino $\left|F=2, m_{F}=2\right\rangle$ e, por contato térmico, esfriar átomos de ${ }^{87} R b$ no estado hiperfino $\left|F=1, m_{F}=-1\right\rangle$, a uma temperatura que induza a condensação dos átomos nesse estado hiperfino.

Um outro tipo de mistura foi obtido em $[26,27]$ condensando átomos no mesmo estado hiperfino, porém com diferentes orientações do momento angular total, $m_{F}$. A técnica usa uma armadilha óptica ao invés da armadilha magnética, pois na armadilha ótica não se tem a seleção da projeção do momento angular para que o átomo seja confinado. Dessa forma, condensou-se átomos de sódio de momento angular total $F=1$ e projeções $m_{F}=-1,0$ e 1 . Para certificar-se que estas seriam as projeções desligou-se a armadilha e aplicou-se um campo magnético constante ocasionando o fenômeno de Stern-Gerlach, isto é, conseguiu-se separar os átomos com diferentes projeções $m_{F}$ [27]. Esses tipos de condensados são denominados de condensados spinoriais.

Em outra experiência, [28], conseguiu-se obter uma mistura de condensados em dois estados espacialmente distintos, usando como armadilha externa um potencial com dois mínimos e observou-se, ao desligar o potencial de confinamento, o fenômeno de interferência entre os dois condensados na evolução temporal desse sistema. Mantendo o potencial de confinamento observou-se, através da interferência dos condensados acoplados, o tunelamento das partículas de um mínimo para outro indicando a existência de um acoplamento de Josephson externo entre os dois estados [29].

Um grupo de vários pesquisadores, tanto teóricos quanto experimentais, propuseram um esquema de dois fótons para popular estados excitados correspondentes a dois estados hiperfinos do átomo [30]. Da idéia surgiu um método de obter misturas de condensados que 
consistia em aprisionar, da maneira usual, partículas num determinado estado hiperfino e com um esquema de dois fótons induzir uma transição para outro estado hiperfino dando um completo acesso de um nível hiperfino para o outro. A transição de dois fótons usada para mudar o estado hiperfino é mostrado na figura 1.1. O mecanismo funciona com a aplicação de uma micro-onda numa freqüência ligeiramente menor do que a diferença de energia entre o estado hiperfino com $F=1$ e $m_{F}=-1(|1,-1\rangle)$ e o estado hiperfino com $F=2$ e $m_{F}=1(|2,1\rangle)$ do ${ }^{87} R b(\approx 6.8 \mathrm{GHz})$, juntamente com um campo magnético de rádio freqüência com $\approx 2 \mathrm{MHz}$. Isso conecta o estado $|1,-1\rangle$ com o estado $|2,1\rangle$ através de um estado intermediário com uma dessintonia de $2.2 \mathrm{MHz}$ do estado $F=2$ e $m_{F}=0$ $(|2,0\rangle)$.

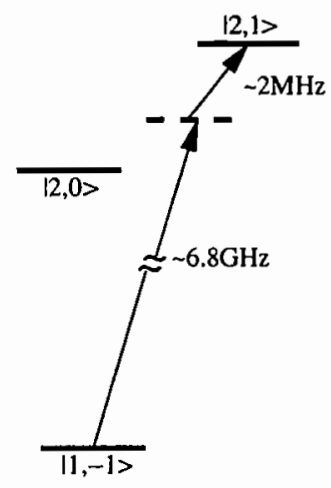

Figura 1.1: O diagrama da transição do estado hiperfino $|1,-1\rangle$ para $|2,1\rangle$.

No caso dessas misturas temos dois papéis diferentes para o campo de radiação. Em um, o laser é usado para transferir átomos do estado hiperfino $|1,-1\rangle$ para o $|2,1\rangle$ e depois desligado. Nesse caso o papel do campo de radiação é estabelecer o estado inicial da mistura de dois condensados mudando a população de átomos de cada estado hiperfino [31]. No outro, caso este que estamos particularmente interessados, as medidas são realizadas com o laser ligado [32] e, portanto, o campo de radiação tem como função acoplar os dois estados, onde o acoplamento é definido como sendo de Josephson interno [18].

Recentemente, conseguiu-se observar condensados heteronucleares, ou seja, condensados de elementos bosônicos distintos como ${ }^{41} K-{ }^{87} R b$ [33]. Nesse caso, conseguiu-se esfriar os átomos de ${ }^{41} K$ até alcançar a temperatura de transição por contato térmico com o condensado de ${ }^{87} R b$. Observou-se, também, misturas de férmions e bósons por exemplo misturas de um gás de Fermi de ${ }^{6} \mathrm{Li}$ com um condensado de ${ }^{7} \mathrm{Li}$, [34], ou com 
um condensado de ${ }^{23} \mathrm{Na},[35]$.

Até então, todas as experiências mencionadas consideram nula a temperatura do sistema. Das poucas experiências que investigam efeitos relacionados com a temperatura, vemos como exemplo que, em condensados com uma componente, mediu-se a dependência com a temperatura da energia e do decaimento das excitações coletivas [36, 37]. Observouse que as energias e os decaimentos dos modos dependem da temperatura, indicando uma significativa interação entre o condensado e a nuvem térmica. Em misturas de condensados não temos conhecimento de trabalhos experimentais sobre os estados de equilíbrio em temperatura finita e nem das excitações coletivas em temperatura nula ou finita.

\subsection{As teorias}

Os condensados produzidos experimentalmente são sistemas confinados, diluídos e fracamente interagentes de gases de átomos bosônicos a uma temperatura tão baixa que pode-se considerar como nula. Gases corn essas propriedades são exemplos de sistemas que são descritos pela teoria de Bogoliubov estendida para sistemas confinados onde supomos que os átomos ocupam o mesmo estado, denominado de função de onda do condensado. Nessa teoria a equação de Gross-Pitaevskii independente do tempo [38, 39, 40] determina os estados de equilíbrio [41, 42, 43]. A dinâmica do sistema é dada pela equação de GrossPitaevskii dependente do tempo [44] e sua linearização em torno do estado fundamental descreve as excitações coletivas de baixa energia $[45,46,47]$ e corresponde as equações de Bogoliubov generalizadas para sistemas confinados. Os cálculos teóricos [47] reproduzem os dados experimentais e sugerem, por exemplo, que as excitações coletivas estão relacionadas com a translação e as oscilações monopolares e quadrupolares da densidade do estado fundamental do condensado [48, 49].

A teoria de Bogoliubov para misturas de condensados, tanto com acoplamento quanto sem, é uma generalização natural da teoria para o sistema de uma componente [18, 50]. Vemos, como exemplo, que para misturas de duas componentes desacopladas, isto é, o número de átomos em cada componente é conservado, temos duas equações de GrossPitaevskii independentes do tempo acopladas [52] que determinam os estados de equilíbrio. De um modo análogo, estende-se as equações de Gross-Pitaevskii dependente do tempo para misturas de condensados $[18,50]$ e sua linearização em torno do estado fundamental 
determina as excitações coletivas do sistema $[53,54,55,56,57]$ e correspondem as equações de Bogoliubov generalizadas para misturas confinadas de condensados.

Em misturas acopladas não há conservação do número de átomos de cada componente da mistura, o que se conserva é o número total de átomos. Nesse caso, a presença de apenas um potencial químico e de um termo que transfere átomos entre os condensados são as únicas diferenças entre as equações. Como anteriormente, os estados de equilíbrio para misturas acopladas são determinados resolvendo as equações de Gross-Pitaevskii independente do tempo $[18,50]$ e sua dinâmica é dada pela equações de Gross-Pitaevskii dependente do tempo [51]. A linearização das equações de Gross-Pitaevskii dependente do tempo em torno do estado fundamental descreve as excitações coletivas [18, 50].

Nas experiências em condensados confinados à temperatura nula, o número de átomos fora dos condensados é desprezível (da ordem de $1 \%$ do número total de átomos) e a teoria de Bogoliubov é válida. Por outro lado, quando a temperatura é diferente de zero e com o seu aumento, o efeito da presença de átomos fora do condensado começa a ser considerável e a teoria de Bogoliubov não pode ser usada.

Uma teoria que leva em consideração a presença de uma nuvem térmica no sistema é a teoria de Hartree-Fock-Bogoliubov a uma temperatura finita [59]. A teoria de HartreeFock-Bogoliubov determina, de forma auto-consistente, os estados de equilíbrio e as excitações coletivas. Porém, no caso de um sistema homogêneo, o espectro de energia das excitações coletivas tem uma lacuna no limite de momento nulo, o que viola o teorema de Hugenholtz-Pines [60], que afirma não haver lacuna no espectro de excitação. Pode-se ir além da teoria de Hartree-Fock-Bogoliubov considerando-se as interações entre pares de quasi-partículas e obter um espectro sem lacuna [61], porém essa teoria é computacionalmente complicada.

Uma outra forma de contornar o problema da lacuna no espectro de excitação foi proposta por Popov [62] e consiste em desprezar de forma ad hoc a contribuição da densidade de pares dos átomos fora do condensado na teoria de Hartree-Fock-Bogoliubov. Essa aproximação foi usada em [63,64] para determinar o perfil da densidade do condensado, a depleção e a dependência com a temperatura das energias de excitação de um gás de átomos bosônicos confinados numa armadilha harmônica. Para temperaturas menores que $60 \%$ da temperatura de transição, os cálculos estão de acordo com os dados experimentais, porém quando a temperatura aproxima-se da temperatura de transição há 
discordância entre os valores calculados e os dados experimentais possivelmente por, essa teoria, não considerar as excitações da nuvem térmica $[64,65,66]$.

A teoria de Hartree-Fock-Bogoliubov-Popov à temperatura finita pode ser estendida para descrever misturas de condensados à temperatura finita. Existem poucos trabalhos teóricos sobre misturas de condensados à temperatura finita. Um exemplo é o estudo, na referência [67], do efeito da temperatura na estabilidade de uma mistura binária de condensados desacoplados.

Do ponto de vista teórico, vários trabalhos estudam as propriedades do sistema de condensados no limite homogêneo. Apesar do caráter qualitativo na comparação entre os cálculos em sistemas homogêneos e em sistemas confinados, as vantagens conceituais e computacionais de trabalhar numa geometria uniforme supera, em larga escala, possíveis imprecisões nesta comparação. Não menos importante, sua simplicidade revela, para qual intervalo de valores dos parâmetros do sistema, novos fenômenos ocorrem, sugerindo uma investigação tanto teórica quanto experimental em sistemas confinados.

Nos atendo à misturas homogêneas de condensados, em temperatura nula e: no limite de gases diluídos, na referência [68] deduz-se a generalização da teoria de Bogoliubov para misturas de condensados desacoplados. Para misturas binárias acopladas, a fração de átomos em cada condensado, a energia de excitação e a estabilidade do sistema são estudadas em trabalhos recentes $[58,69,70]$.

Em especial, a referência [70] mostra como reduzir a equação de Gross-Pitaevskii para os estados de equilíbrio a uma equação para a fração relativa de átomos nos condensados que dependem de dois parâmetros de controle definidos pela combinação dos parâmetros do sistema. Na determinação dos estados de equilíbrio mostra-se que existe uma região no espaço de parâmetros de controle onde tem-se uma estrutura de bi-estabilidade, ou seja, o sistema pode estar em dois mínimos locais. Como conseqüência da bi-estabilidade ocorre o fenômeno de histerese, ou seja, o estado do sistema depende de sua história, e o de colapso de um dos estados de equilíbrio, com a possibilidade de mudanças abruptas da fração relativa de átomos nos condensados. Para examinar até que ponto o termo de acoplamento de Josephson influencia as propriedades da mistura de condensados, calcula-se a depleção dos condensados, o espectro de energia de excitações coletivas, sua estabilidade, a lacuna e a velocidade do som. Os cálculos dessas propriedades físicas indicam que o acoplamento de Josephson interno influencia a física de muitos corpos da mistura de condensados. 
No trabalho de tese, examinamos como as propriedades de uma mistura binária de condensados na presença de um termo de acoplamento de Josephson interno dependem da temperatura. Fizemos esse estudo no contexto da teoria de Hartree-Fock-BogoliubovPopov a uma temperatura finita e generalizada para descrever misturas homogêneas de condensados acoplados. Investigamos o comportamento da bi-estabilidade do sistema como função da temperatura. Calculamos quantidades físicas tais como frações de átomos condensados, lacuna do espectro de energia das excitações coletivas e velocidade do som do sistema para elucidar o papel da temperatura nas propriedades de misturas coerentes de condensados.

\subsection{Escopo da tese}

A tese está organizada do seguinte modo:

No capítulo 2 utilizamos o princípio de máxima entropia para deduzir as equações de Hartree-Fock-Bogoliubov para um sistema homogêneo de misturas de condensados com acoplamento interno de Josephson à temperatura finita. Para contornar o bem conhecido defeito da teoria de Hartree-Fock-Bogoliubov, na qual o espectro de energia das excitações coletivas tem uma lacuna no limite de momento nulo, mostramos como definir a aproximação de Popov para misturas acopladas de condensados. Seguindo a referência [70] reduzimos a equação de Gross-Pitaevskii a uma equação para a fração relativa de átomos condensados com os parâmetros de controle dependentes da temperatura. Deduzimos expressões explícitas para energias das excitações coletivas, a lacuna e a velocidade do som.

No capítulo 3 apresentamos e discutimos os resultados dos cálculos, como função da temperatura, da população de átomos nos condensados, da lacuna e da velocidade do som e examinamos a dependência com a temperatura da estrutura de bi-estabilidade determinada na referência [70].

No capítulo 4 finalizamos a tese com nossas conclusões. Deduções mais formais foram anexadas nos apêndices A e B para que o leitor não se perca na leitura. 


\section{Capítulo 2}

\section{A teoria e suas aproximações}

"Os corpos distinguem-se uns dos outros em razão do movimento e do repouso, da rapidez e da lentidão, e não em razão da substância."

Espinosa

\subsection{O hamiltoniano do sistema}

Supomos que o sistema seja composto por $N$ átomos em 2 estados hiperfinos distingǘveis, $|1\rangle$ e $|2\rangle$, confinados em uma caixa de volume $\Omega$ e interagindo com um campo de radiação que acople os dois estados hiperfinos. Por ser homogêneo, escrevemos o hamiltoniano do sistema em segunda quantização em termos dos operadores de criação (de destruição), $a_{\alpha \vec{k}}^{\dagger}\left(a_{\alpha \vec{k}}\right)$, de um átomo com momento $\vec{k}$, no $\alpha$-ésimo estado hiperfino, onde $\alpha=1,2$. Desse modo, o hamiltoniano na sua forma mais geral é dada por:

$$
\begin{aligned}
H & =\sum_{\alpha} \sum_{\vec{k}} \frac{\hbar^{2} k^{2}}{2 m} a_{\alpha \vec{k}}^{\dagger} a_{\alpha \vec{k}}+\sum_{\alpha \beta} \sum_{\vec{k}} \Lambda_{\alpha \beta} a_{\alpha \vec{k}}^{\dagger} a_{\beta \vec{k}}+ \\
& +\frac{1}{2 \Omega} \sum_{\alpha \beta} \sum_{\vec{k} \vec{k}^{\prime} \vec{q}} \tilde{V}_{\alpha \beta}(\vec{q}) a_{\alpha(\vec{k}+\vec{q})}^{\dagger} a_{\beta(\vec{k}-\vec{q})}^{\dagger} a_{\alpha \vec{k}} a_{\beta \vec{k}^{\prime}}
\end{aligned}
$$

Nesse hamiltoniano, o primeiro termo corresponde à energia cinética do sistema. $\mathrm{O}$ segundo termo descreve a interação entre os átomos e o campo de radiação, onde $\Lambda_{\alpha \beta}$, é um elemento da matriz real e simétrica

$$
\Lambda=\left(\begin{array}{cc}
\frac{\delta}{2} & J \\
J & -\frac{\delta}{2}
\end{array}\right) .
$$


Os elementos diagonais dependem da dessintonia $\delta$ e introduzem uma energia de partícula independente efetiva para os estados hiperfinos. Os elementos não diagonais são as intensidades do termo de acoplamento de Josephson, $J$, que permite a transição atômica de uma espécie para outra conservando o momento do átomo.

O último termo corresponde a interação binária entre os átomos do sistema. O elemento de matriz que aparece na expressão do operador de dois corpos, $\tilde{V}_{\alpha \beta}(\vec{q})$, é dado pela transformada de Fourier do potencial entre os átomos nos estados hiperfinos $\alpha$ e $\beta$,

$$
\tilde{V}_{\alpha \beta}(\vec{q})=\int V_{\alpha \beta}(\vec{r}) e^{i \vec{q} \cdot \vec{r}} d^{3} \vec{r}
$$

onde $V_{\alpha \beta}(\vec{r})$ é o potencial de interação entre os átomos. Como o potencial de interação é hermiteano e invariante por reflexão espacial segue que a transformada de Fourier do potencial, $\tilde{V}_{\alpha \beta}(\vec{q})$, é uma função real, par do momento transferido, $\vec{q}$, e simétrica nos índices hiperfinos.

Observamos que, nesse sistema, o operador número de partículas

$$
N=\sum_{\alpha} \sum_{\vec{k}} a_{\alpha \vec{k}}^{\dagger} a_{\alpha \vec{k}}
$$

comuta com o hamiltoniano (2.1) fazendo com que o número total de partículas seja conservado. Entretanto, o número de partículas em cada estado hiperfino, cujo operador é $N_{\alpha}=\sum_{\vec{k}} a_{\alpha \vec{k}}^{\dagger} a_{\alpha \vec{k}}$, não se conserva devido ao termo de acoplamento de Josephson.

Em um sistema cujo número de partículas é grande, $N \gg 1$, podemos assumir a existência de dois estados quânticos coerentes de momento nulo correspondendo um para cada estado hiperfino, e que esse estado quântico coerente seja auto-estado dos operadores de aniquilação de momento nulo, $a_{\alpha 0}$, com autovalor $z_{\alpha}$, a qual chamamos de função de onda do condensado da $\alpha$-ésima espécie. A introdução dessa função de onda no problema é feita deslocando os operadores de criação e aniquilação dessas partículas por essas quantidades,

$$
\left\{\begin{array}{l}
a_{\alpha \vec{k}}^{\dagger}=c_{\alpha \vec{k}}^{\dagger}+z_{\alpha}^{*} \delta_{\vec{k}, 0} \\
a_{\alpha \vec{k}}=c_{\alpha \vec{k}}+z_{\alpha} \delta_{\vec{k}, 0}
\end{array},\right.
$$

assim os novos operadores de aniquilação têm como vácuo os seus respectivos estados coerentes.

Ao fazermos a transformação (2.5), expandimos o hamiltoniano (2.1) na ordem normal dos operadores deslocados

$$
H=H_{0}+H_{1}+H_{2}+H_{3}+H_{4}
$$


onde

$$
\begin{aligned}
& H_{0}=\sum_{\alpha \beta} \Lambda_{\alpha \beta} z_{\alpha}^{*} z_{\beta}+\frac{1}{2 \Omega} \sum_{\alpha, \beta} \tilde{V}_{\alpha \beta}(0)\left|z_{\alpha}\right|^{2}\left|z_{\beta}\right|^{2}, \\
& H_{1}=\sum_{\alpha \beta}\left(\Lambda_{\alpha \beta} z_{\beta}^{*}+\frac{\tilde{V}_{\alpha \beta}(0)}{\Omega}\left|z_{\beta}\right|^{2} z_{\alpha}^{*}\right) c_{\alpha 0}+\sum_{\alpha \beta}\left(\Lambda_{\alpha \beta} z_{\beta}+\frac{\tilde{V}_{\alpha \beta}(0)}{\Omega}\left|z_{\beta}\right|^{2} z_{\alpha}\right) c_{\alpha 0}^{\dagger} \\
& H_{2}=\sum_{\alpha} \sum_{\vec{k}}\left(\frac{\hbar^{2} k^{2}}{2 m}+\frac{1}{\Omega} \sum_{\beta} \tilde{V}_{\alpha \beta}(0)\left|z_{\beta}\right|^{2}\right) c_{\alpha \vec{k}}^{\dagger} c_{\alpha \vec{k}}+\sum_{\alpha \beta} \sum_{\vec{k}}\left(\Lambda_{\alpha \beta}+\frac{\tilde{V}_{\alpha \beta}(\vec{k})}{\Omega} z_{\alpha} z_{\beta}^{*}\right) c_{\alpha \vec{k}}^{\dagger} c_{\beta \vec{k}}+ \\
& +\frac{1}{2 \Omega} \sum_{\alpha, \beta} \sum_{\vec{k}} \tilde{V}_{\alpha \beta}(\vec{k})\left(z_{\alpha} z_{\beta} c_{\alpha \vec{k}}^{\dagger} c_{\beta-\vec{k}}^{\dagger}+z_{\alpha}^{*} z_{\beta}^{*} c_{\alpha \vec{k}} c_{\beta-\vec{k}}\right), \\
& H_{3}=\frac{1}{\Omega} \sum_{\alpha \beta} \sum_{\vec{k}, \vec{k}^{\prime}} \tilde{V}_{\alpha \beta}\left(\vec{k}^{\prime}\right)\left(z_{\alpha}^{*} c_{\beta\left(\vec{k}+\vec{k}^{\prime}\right)}^{\dagger} c_{\beta \vec{k}} c_{\alpha \vec{k}^{\prime}}+z_{\alpha} c_{\beta \vec{k}}^{\dagger} c_{\alpha \vec{k}^{\prime}}^{\dagger} c_{\beta\left(\vec{k}+\vec{k}^{\prime}\right)}\right)
\end{aligned}
$$

e, finalmente,

$$
H_{4}=\frac{1}{2 \Omega} \sum_{\alpha \beta} \sum_{\vec{k} \vec{k}^{\prime} \vec{q}} \tilde{V}_{\alpha \beta}(\vec{q}) c_{\alpha(\vec{k}+\vec{q})}^{\dagger} c_{\beta\left(\vec{k}^{\prime}-\vec{q}\right)}^{\dagger} c_{\alpha \vec{k}^{\prime}} c_{\beta \vec{k}^{\prime}}
$$

Em termos dos operadores deslocados de criação e de destruição, o operador de número pode ser escrito como

$$
N=\sum_{\alpha}\left(\left|z_{\alpha}\right|^{2}+z_{\alpha}^{*} c_{\alpha 0}+c_{\alpha 0}^{\dagger} z_{\alpha}+\sum_{\vec{k}} c_{\alpha \vec{k}}^{\dagger} c_{\alpha \vec{k}}\right)
$$

e esse operador comuta com o hamiltoniano mostrando a conservação do número de átomos.

Notamos que a ordem normal estabelece uma hierarquia nos termos da expansão: $H_{0}$ envolve somente partículas no condensado de átomos com momento zero, $H_{1}$ envolve uma partícula fora do condensado, $H_{2}$ duas, $H_{3}$ três e, finalmente, $H_{4}$ envolve somente partículas fora do condensado.

Uma aproximação freqüentemente usada e introduzida por Bogoliubov em [14] consiste em desprezar os termos de terceira e quarta ordem no número de operadores de criação e destruição, $H_{3}$ e $H_{4}$, sob o pretexto de que esses termos envolvem um número de partículas fora do condensado maior do que os outros termos e, portanto, desprezíveis em relação à $H_{0}, H_{1}$ e $H_{2}$. Com efeito, vemos nesta aproximação a necessidade do sistema ter uma 
fração considerável de partículas no condensado. Uma outra constatação é que a teoria de Bogoliubov conduz-nos a um hamiltoniano que não comuta com o operador número, ou seja, quebra a simetria do número de partículas no sistema.

Quando a temperatura é diferente de zero, sabemos que o número de partículas fora do condensado torna-se importante, limitando, portanto, o uso da teoria de Bogoliubov. Uma teoria que leve em consideração a presença de átomos fora dos condensados é a teoria de Hartree-Fock-Bogoliubov onde considera-se os termos desprezados da teoria de Bogoliubov, $\mathrm{H}_{3}$ e $\mathrm{H}_{4}$. Assim, motivados por descrever o sistema à temperatura diferente de zero, desenvolvemos a teoria de Hartree-Fock-Bogoliubov para misturas através de uma teoria variacional considerando todos os termos da expansão no hamiltoniano.

\subsection{Princípio de entropia máxima}

O sistema a temperatura não nula pode ser descrito por um operador de densidade estatística, $D$, que atua no espaço de Fock. Esse operador tem como característica ser hermiteano, definido positivo e de traço igual a unidade. Em termos de seus autovetores, um operador de densidade estatística é representado na forma

$$
D=\sum_{i} p_{i}|i\rangle\langle i|
$$

com $p_{i} \geq 0$ e $\operatorname{tr} D=1=\sum_{i} p_{i}$. Identificamos os autovalores, $p_{i}$, como sendo a probabilidade de encontrar o sistema no estado de Fock $|i\rangle$. Um caso especial é quando o sistema encontra-se somente num estado $|i\rangle$ (estado puro), isso corresponde a $p_{j}=\delta_{i j}$. Nesse caso, o operador $D$ se torna um projetor, $D=|i\rangle\langle i|$, e, como tal, satisfaz a condição de $D=D^{2}$.

O significado físico desse operador manifesta-se no cálculo da média térmica de qualquer observável. Sabendo que o sistema é descrito pelo operador de densidade estatística $D$, a média térmica de qualquer observável, $A$, é definida como

$$
\langle A\rangle=\operatorname{Tr} D A=\sum_{i} p_{i}\langle i|A| i\rangle
$$

onde o traço é tomado sobre todo espaço de Fock.

Em mecânica estatística, uma das maneiras de obter o operador densidade estatística é buscar um que maximize a entropia do sistema. Para isso, devemos primeiro definir a 
função entropia como a média térmica do logarítimo do operador densidade estatística,

$$
S=-k_{B} \operatorname{Tr} D \ln D
$$

onde $k_{B}$ é a constante de Boltzman. Dessa forma, usamos o princípio de máxima entropia e o método variacional para determinar um operador densidade de equilíbrio que torne a entropia um ponto de máximo, ou seja, um operador $D_{e q}$ tal que $\delta S\left(D_{e q}\right)=0$, sujeito as condições adicionais que o sistema tenha uma energia média, $E=\langle H\rangle$, e um número médio de partículas $N=\langle N\rangle$ conhecidos. Posto dessa forma, devemos minimizar o funcional $^{1}$

$$
\phi(D)=\operatorname{Tr} D\left[\frac{1}{\beta} \ln D+H-\mu N\right]
$$

onde $\beta$ e $\mu$ são multiplicadores de Lagrange $\operatorname{com} \beta=1 / k_{B} T$.

Considerando a variação em (2.16) temos

$$
\delta \phi=\frac{1}{\beta} \operatorname{Tr} \delta D[\ln D+1+\lambda+\beta(H-\mu N)]
$$

onde $\lambda$ assegura a normalização do operador densidade estatística, $\operatorname{Tr} D=1$. Como $\delta \phi$ é nulo em $D=D_{e q}$ e como $\delta D$ é uma variação arbitrária, vemos que

$$
\ln D_{e q}=-1-\lambda-\beta(H-\mu N)
$$

e com isso, podemos afirmar

$$
D_{e q}=\frac{1}{\Xi} e^{-\beta(H-\mu N)}
$$

O traço do operador $D_{e q}$ tem que ser igual a 1 , com isso é possível identificar a constante $\Xi$ como a função de partição grão-canônica dado por

$$
\Xi=\operatorname{Tr} e^{-\beta(H-\mu N)}
$$

\subsection{Determinação do hamiltoniano grão-canônico e- fetivo}

O operador densidade estatística (2.19) é exato, contudo calcular a média térmica usando esse operador é impossível pois não conhecemos uma base no espaço de Fock

\footnotetext{
${ }^{1} \mathrm{O}$ funcional $\phi(D)$ é conhecido como potencial termodinâmico, pois recupera o caso em que $\phi(D)=$ $E-\mu N-T S$.
} 
que torne $H-\mu N$ diagonal. Para contornar esse problema usamos a teoria de HartreeFock-Bogoliubov que aproxima o operador densidade estatística por um operador teste da forma

$$
D=\frac{e^{-\beta K_{e f}}}{\operatorname{Tr} e^{-\beta K_{e f}}}
$$

onde $K_{e f}$ é um hamiltoniano na forma quadrática nos operadores deslocados $c_{\alpha \vec{k}}^{\dagger}$ e $c_{\alpha \vec{k}}$,

$$
K_{e f}=\sum_{\alpha, \beta} \sum_{\vec{k}} h_{\alpha \beta}(\vec{k}) c_{\alpha \vec{k}}^{\dagger} c_{\beta \vec{k}}+\frac{1}{2} \sum_{\alpha, \beta} \sum_{\vec{k}}\left(\Delta_{\alpha \beta}(\vec{k}) c_{\alpha \vec{k}}^{\dagger} c_{\beta-\vec{k}}^{\dagger}+\Delta_{\alpha \beta}^{*}(\vec{k}) c_{\alpha \vec{k}} c_{\beta-\vec{k}}\right)
$$

cujos coeficientes, $h_{\alpha \beta}(\vec{k}), \Delta_{\alpha \beta}(\vec{k})$ e $\Delta_{\alpha \beta}^{*}(\vec{k})$, são parâmetros determinados através do princípio variacional.

Como $K_{e f}$ tem que ser um operador hermiteano, a matriz, $h(\vec{k})$, cujos elementos são os $h_{\alpha \beta}(\vec{k})$, é uma matriz hermiteana e a matriz, $\Delta(\vec{k})$, cujos elementos são os $\Delta_{\alpha \beta}(\vec{k})$, é uma matriz simétrica.

O hamiltoniano efetivo é determinado através do método variacional e consiste em procurar valores de $h_{\alpha \beta}(\vec{k}), \Delta_{\alpha \beta}(\vec{k})$ e $z_{\alpha}$ que minimizem o funcional

$$
\phi=\operatorname{Tr} D\left[\frac{1}{\beta} \ln D+H-\mu N\right] .
$$

Dado (2.21), mostrou-se na referência [71] que (2.23) pode ser reescrito como

$$
\phi=-\frac{\ln \Xi_{e f}}{\beta}-\operatorname{Tr} D K_{e f}+\operatorname{Tr} D(H-\mu N)
$$

onde $\Xi_{e f}=\operatorname{Tr} e^{-\beta K_{e f}}$. Ao minimizar (2.24), $\delta \phi=0$, temos a expressão

$$
\operatorname{Tr} \delta D K_{e f}=\delta \operatorname{Tr} D(H-\mu N)
$$

que relaciona a variação da média de $H-\mu N$ com a variação da média térmica do hamiltoniano efetivo, $K_{e f}$, considerando os parâmetros variacionais desse hamiltoniano efetivo fixo na variação.

Com o propósito de calcular a média térmica de $H-\mu N$, fatoramos as médias de produtos de quatro operadores usando o teorema de Wick demonstrado no apêndice $\mathrm{A}$. Assim, vemos que a média térmica pode ser escrita como a soma de três termos [71],

$$
\operatorname{Tr} D(H-\mu N)=E_{c}+E_{d}+V_{c d}
$$


onde

$$
E_{c}=\sum_{\alpha}(-\mu)\left|z_{\alpha}\right|^{2}+\frac{1}{2 \Omega} \sum_{\alpha, \beta} \tilde{V}_{\alpha \beta}(0)\left|z_{\alpha}\right|^{2}\left|z_{\beta}\right|^{2}+\sum_{\alpha, \beta} \Lambda_{\alpha \beta} z_{\alpha}^{*} z_{\beta},
$$

corresponde a contribuição para a média térmica dos átomos condensados,

$$
\begin{aligned}
E_{d} & =\sum_{\alpha} \sum_{\vec{k}}\left(\frac{\hbar^{2} k^{2}}{2 m}-\mu\right)\left\langle c_{\alpha \vec{k}}^{\dagger} c_{\alpha \vec{k}}\right\rangle+\sum_{\alpha, \beta} \sum_{\vec{k}} \Lambda_{\alpha \beta}\left\langle c_{\alpha \vec{k}}^{\dagger} c_{\beta \vec{k}}\right\rangle+ \\
& +\frac{1}{2 \Omega} \sum_{\alpha, \beta} \sum_{\vec{k} \vec{k}^{\prime}} \tilde{V}_{\alpha \beta}(0)\left\langle c_{\alpha \vec{k}^{\prime}}^{\dagger} c_{\alpha \vec{k}^{\prime}}\right\rangle\left\langle c_{\beta \vec{k}}^{\dagger} c_{\beta \vec{k}}\right\rangle+\frac{1}{2 \Omega} \sum_{\alpha, \beta} \sum_{\vec{k} \vec{k}^{\prime}} \tilde{V}_{\alpha \beta}\left(\vec{k}^{\prime}-\vec{k}\right)\left\langle c_{\alpha \vec{k}^{\prime}}^{\dagger} c_{\beta \vec{k}^{\prime}}\right\rangle\left\langle c_{\beta \vec{k}}^{\dagger} c_{\alpha \vec{k}}\right\rangle+ \\
& +\frac{1}{2 \Omega} \sum_{\alpha, \beta} \sum_{\vec{k} \vec{k}^{\prime}} \tilde{V}_{\alpha \beta}\left(\vec{k}^{\prime}-\vec{k}\right)\left\langle c_{\alpha \vec{k}^{\prime}}^{\dagger} c_{\beta-\vec{k}^{\prime}}^{\dagger}\right\rangle\left\langle c_{\alpha \vec{k}} c_{\beta-\vec{k}}\right\rangle
\end{aligned}
$$

corresponde a contribuição dos átomos fora do condensado, e

$$
\begin{aligned}
V_{c d} & =\sum_{\alpha, \beta} \sum_{\vec{k}}\left(\frac{\tilde{V}_{\alpha \beta}(0)}{\Omega}\left|z_{\beta}\right|^{2}\right)\left\langle c_{\alpha \vec{k}}^{\dagger} c_{\alpha \vec{k}}\right\rangle+\sum_{\alpha, \beta} \sum_{\vec{k}}\left(\frac{\tilde{V}_{\alpha \beta}(\vec{k})}{\Omega} z_{\beta}^{*} z_{\alpha}\right)\left\langle c_{\alpha \vec{k}}^{\dagger} c_{\beta \vec{k}}\right\rangle+ \\
& +\frac{1}{2 \Omega} \sum_{\alpha, \beta} \sum_{\vec{k}} \tilde{V}_{\alpha \beta}(\vec{k})\left(z_{\alpha} z_{\beta}\left\langle c_{\alpha \vec{k}}^{\dagger} c_{\beta-\vec{k}}^{\dagger}\right\rangle+z_{\alpha}^{*} z_{\beta}^{*}\left\langle c_{\alpha \vec{k}} c_{\beta-\vec{k}}\right\rangle\right)
\end{aligned}
$$

é o termo de interação entre os átomos condensados e os não condensados.

Do princípio variacional (2.25) somos capazes de deduzir os parâmetros do hamiltoniano efetivo (2.21) derivando a média de $H-\mu N$ em relação as médias térmicas dos produtos bilineares de operadores deslocados. Desta forma, vemos que o termo $h_{\alpha \beta}(\vec{k})$ é dado por

$$
h_{\alpha \beta}(\vec{k})=\frac{\partial \operatorname{Tr} D(H-\mu N)}{\partial\left\langle c_{\alpha \vec{k}}^{\dagger} c_{\beta \vec{k}}\right\rangle},
$$

ou seja,

$$
\begin{aligned}
h_{\alpha \beta}(\vec{k}) & =\left(\frac{\hbar^{2} k^{2}}{2 m}-\mu+\frac{1}{\Omega} \sum_{\alpha^{\prime}} \tilde{V}_{\alpha \alpha^{\prime}}(0)\left[\left|z_{\alpha^{\prime}}\right|^{2}+\sum_{\vec{k}^{\prime}}\left\langle c_{\alpha^{\prime} \vec{k}^{\prime}}^{\dagger} c_{\alpha^{\prime} \vec{k}^{\prime}}\right\rangle\right]\right) \delta_{\alpha, \beta}+ \\
& +\Lambda_{\alpha \beta}+\frac{1}{\Omega} \tilde{V}_{\alpha \beta}(\vec{k}) z_{\beta}^{*} z_{\alpha}+\frac{1}{\Omega} \sum_{\vec{k}^{\prime}} \tilde{V}_{\alpha \beta}\left(\vec{k}-\vec{k}^{\prime}\right)\left\langle c_{\beta \overrightarrow{k^{\prime}}}^{\dagger} c_{\alpha \vec{k}^{\prime}}\right\rangle,
\end{aligned}
$$

e, por sua vez, o parâmetro do emparelhamento é dado por

$$
\Delta_{\alpha \beta}(\vec{k})=\frac{\partial \operatorname{Tr} D(H-\mu N)}{\partial\left\langle c_{\alpha \vec{k}}^{\dagger} c_{\beta \vec{k}}^{\dagger}\right\rangle},
$$

ou seja,

$$
\Delta_{\alpha \beta}(\vec{k})=\frac{1}{\Omega} \tilde{V}_{\alpha \beta}(\vec{k}) z_{\alpha} z_{\beta}+\frac{1}{\Omega} \sum_{\vec{k}^{\prime}} \tilde{V}_{\alpha \beta}\left(\vec{k}-\vec{k}^{\prime}\right)\left\langle c_{\alpha \vec{k}^{\prime}} c_{\beta-\vec{k}^{\prime}}\right\rangle .
$$


De (2.31) concluímos que $h_{\alpha \beta}(\vec{k})=h_{\beta \alpha}^{*}(\vec{k})$, ou seja, a matriz, $h(\vec{k})$, cujos elementos são os coeficientes $h_{\alpha \beta}(\vec{k})$, é hermiteana, consistente com a hipótese do hamiltoniano efetivo ser hermiteano. De (2.33) vem que $\Delta_{\alpha \beta}(\vec{k})$ é simétrico, com isso, a matriz, $\Delta(\vec{k})$, cujos elementos são os coeficientes $\Delta_{\alpha \beta}(\vec{k})$, é simétrica, concordando com o fato do hamiltoniano efetivo ser hermiteano.

Notamos que o hamiltoniano efetivo (2.22) não comuta com o operador número, isso implica, da mesma forma que na teoria de Bogoliubov, na quebra da simetria de número. Entretanto, vemos que os coeficientes de (2.22) contém, de sua dedução, contribuições de $H_{4}$ que na teoria de Bogoliubov foram desprezados. Esperamos, com isso, que a aproximação por um hamiltoniano efetivo (2.22) seja mais geral que a teoria de Bogoliubov e que permita-nos descrever o sistema com uma depleção não desprezível.

\subsection{Equações de Hartree-Fock-Bogoliubov dependen- te da temperatura}

Deduzimos as equações de Hartree-Fock-Bogoliubov de maneira semelhante a descrita por de Gennes na referência [72] para um sistema supercondutor.

Conhecidos os coeficientes do hamiltoniano efetivo (2.22) determinamos, a partir deles, um hamiltoniano efetivo de quasi-partículas livres,

$$
K_{e f}=\sum_{\gamma} \sum_{\vec{k}} E_{\gamma}(\vec{k}) b_{\gamma \vec{k}}^{\dagger} b_{\gamma \vec{k}}
$$

Isso é possível pois sabemos que um hamiltoniano bosônico quadrático nos operadores de criação e aniquilação pode ser diagonalizado por uma transformação canônica nos operadores deslocados, $c_{\alpha \vec{k}}^{\dagger}\left(c_{\alpha \vec{k}}\right)$,

$$
\left\{\begin{array}{l}
c_{\alpha \vec{k}}=\sum_{\gamma}\left[u_{\alpha \gamma}(\vec{k}) b_{\gamma \vec{k}}+v_{\alpha \gamma}^{*}(\vec{k}) b_{\gamma-\vec{k}}^{\dagger}\right] \\
c_{\alpha \vec{k}}^{\dagger}=\sum_{\gamma}\left[u_{\alpha \gamma}^{*}(\vec{k}) b_{\gamma \vec{k}}^{\dagger}+v_{\alpha \gamma}(\vec{k}) b_{\gamma-\vec{k}}\right]
\end{array}\right.
$$

onde os coeficientes $u_{\alpha \beta}(\vec{k})$ e $v_{\alpha \beta}(\vec{k})$ transformam o hamiltoniano efetivo (2.22) no hamiltoniano efetivo de quasi-partículas livres (2.34). Essa transformação é conhecida por transformação de Bogoliubov. Como a transformação é canônica devemos impor as seguintes regras de comutação para os novos operadores,

$$
\left\{\begin{array}{l}
{\left[b_{\alpha \vec{k}}, b_{\alpha^{\prime} \vec{k}^{\prime}}^{\dagger}\right]=\delta_{\alpha, \alpha^{\prime}} \delta_{\vec{k}, \vec{k}^{\prime}}} \\
{\left[b_{\alpha \vec{k}}, b_{\alpha^{\prime} \vec{k}^{\prime}}\right]=0}
\end{array}\right.
$$


Assim, da equação (2.35) vemos que

$$
\begin{aligned}
{\left[c_{\alpha \vec{k}}, c_{\beta \vec{k}^{\prime}}^{\dagger}\right] } & =\sum_{\gamma \eta}\left\{u_{\alpha \gamma}(\vec{k}) u_{\beta \eta}^{*}\left(\vec{k}^{\prime}\right)\left[b_{\gamma \vec{k}}, b_{\eta \vec{k}^{\prime}}^{\dagger}\right]+v_{\alpha \gamma}^{*}(\vec{k}) v_{\beta \eta}\left(\vec{k}^{\prime}\right)\left[b_{\gamma-\vec{k}}^{\dagger}, b_{\eta-\vec{k}^{\prime}}\right]+\right. \\
& \left.+u_{\alpha \gamma}(\vec{k}) v_{\beta \eta}\left(\vec{k}^{\prime}\right)\left[b_{\gamma \vec{k}}, b_{\eta-\vec{k}^{\prime}}\right]+v_{\alpha \gamma}^{*}(\vec{k}) u_{\beta \eta}^{*}\left(\vec{k}^{\prime}\right)\left[b_{\gamma-\vec{k}}^{\dagger}, b_{\eta \vec{k}^{\prime}}^{\dagger}\right]\right\}=\delta_{\alpha, \beta} \delta_{\vec{k}, \vec{k}^{\prime}}
\end{aligned}
$$

com isso, e das condições (2.36), temos que

$$
\sum_{\gamma} u_{\alpha \gamma}(\vec{k}) u_{\beta \gamma}^{*}(\vec{k})-v_{\alpha \gamma}^{*}(\vec{k}) v_{\beta \gamma}(\vec{k})=\delta_{\alpha, \beta},
$$

chegando assim, a uma condição de completeza para os coeficientes da transformação (2.35).

Do mesmo modo, vemos que os comutadores

$$
\begin{aligned}
{\left[c_{\alpha \vec{k}}, c_{\beta \vec{k}^{\prime}}\right] } & =\sum_{\gamma \eta}\left\{u_{\alpha \gamma}(\vec{k}) u_{\beta \eta}\left(\vec{k}^{\prime}\right)\left[b_{\gamma \vec{k}}, b_{\eta \vec{k}^{\prime}}\right]+u_{\alpha \gamma}(\vec{k}) v_{\beta \eta}^{*}\left(\vec{k}^{\prime}\right)\left[b_{\gamma \vec{k}}, b_{\eta-\vec{k}^{\prime}}^{\dagger}\right]+\right. \\
& \left.+v_{\alpha \gamma}^{*}(\vec{k}) u_{\beta \eta}\left(\vec{k}^{\prime}\right)\left[b_{\gamma-\vec{k}}^{\dagger}, b_{\eta \vec{k}^{\prime}}\right]+v_{\alpha \gamma}^{*}(\vec{k}) v_{\beta \eta}^{*}\left(\vec{k}^{\prime}\right)\left[b_{\gamma-\vec{k}}^{\dagger}, b_{\eta \vec{k}^{\prime}}^{\dagger}\right]\right\}=0
\end{aligned}
$$

são nulos, e, de (2.36), temos

$$
\sum_{\gamma} u_{\alpha \gamma}(\vec{k}) v_{\beta \gamma}^{*}(\vec{k})-v_{\alpha \gamma}^{*}(\vec{k}) u_{\beta \gamma}(\vec{k})=0
$$

onde usamos a propriedade de que o sistema é invariante por reflexão espacial, implicando em $u_{\alpha \beta}(\vec{k})$ e $v_{\alpha \beta}(\vec{k})$ serem funções pares de $\vec{k}$.

Agora, vamos determinar as equações de Hartree-Fock-Bogoliubov usando as propriedades dos comutadores.

O comutador do hamiltoniano efetivo de quasi-partícula, (2.34), com o operador deslocado $c_{\alpha \vec{k}}$ é

$$
\begin{aligned}
{\left[K_{e f}, c_{\alpha \vec{k}}\right] } & =\sum_{\beta} u_{\alpha \beta}(\vec{k})\left[K_{e f}, b_{\beta \vec{k}}\right]+v_{\alpha \beta}^{*}(\vec{k})\left[K_{e f}, b_{\beta-\vec{k}}^{\dagger}\right] \\
& =-\sum_{\beta} E_{\beta}(\vec{k})\left[u_{\alpha \beta}(\vec{k}) b_{\beta \vec{k}}-v_{\alpha \beta}^{*}(\vec{k}) b_{\beta-\vec{k}}^{\dagger}\right]
\end{aligned}
$$

Isso se deve ao fato do comutador do operador transformado $b_{\alpha \vec{k}}$ com o hamiltoniano efetivo de quasi-partícula ser dado por

$$
\left[K_{e f}, b_{\alpha \vec{k}}\right]=-E_{\alpha}(\vec{k}) b_{\alpha \vec{k}}
$$

e da mesma forma o comutador do operador transformado $b_{\alpha \vec{k}}^{\dagger}$ com o hamiltoniano efetivo de quasi-partícula seja

$$
\left[K_{e f}, b_{\alpha \vec{k}}^{\dagger}\right]=E_{\alpha}(\vec{k}) b_{\alpha \vec{k}}^{\dagger}
$$


Como o hamiltoniano efetivo é hermiteano, vemos, imediatamente, que o comutador do hamiltoniano efetivo de quasi-partículas com o operador deslocado $c_{\alpha \vec{k}}^{\dagger}$ é dado por

$$
\left[K_{e f}, c_{\alpha \vec{k}}^{\dagger}\right]=\sum_{\beta} E_{\beta}(\vec{k})\left[u_{\alpha \beta}^{*}(\vec{k}) b_{\beta \vec{k}}^{\dagger}-v_{\alpha \beta}(\vec{k}) b_{\beta-\vec{k}}\right]
$$

Por outro lado, calculando os mesmos comutadores usando a expressão do hamiltoniano efetivo, equação (2.22), temos

$$
\begin{aligned}
{\left[K_{e f}, c_{\alpha \vec{k}}\right] } & =\sum_{\beta, \gamma} \sum_{\vec{k}^{\prime}} h_{\beta \gamma}\left(\vec{k}^{\prime}\right)\left[c_{\beta \vec{k}^{\prime}}^{\dagger} c_{\gamma-\vec{k}^{\prime}}, c_{\alpha \vec{k}}\right]+ \\
& +\frac{1}{2} \sum_{\beta, \gamma} \sum_{\vec{k}^{\prime}}\left(\Delta_{\beta \gamma}\left(\vec{k}^{\prime}\right)\left[c_{\beta \vec{k}^{\prime}}^{\dagger} c_{\gamma-\vec{k}^{\prime}}^{\dagger}, c_{\alpha \vec{k}}\right]+\Delta_{\beta \gamma}^{*}\left(\vec{k}^{\prime}\right)\left[c_{\beta \vec{k}^{\prime}} c_{\gamma-\vec{k}^{\prime}}, c_{\alpha \vec{k}}\right]\right)
\end{aligned}
$$

Como os operadores deslocados são operadores bosônicos, segue que

$$
\left[K_{e f}, c_{\alpha \vec{k}}\right]=-\sum_{\alpha^{\prime}}\left[h_{\alpha \alpha^{\prime}}(\vec{k}) c_{\alpha^{\prime} \vec{k}}+\Delta_{\alpha \alpha^{\prime}}(\vec{k}) c_{\alpha^{\prime}-\vec{k}}^{\dagger}\right]
$$

Da mesma forma vemos que

$$
\left[K_{e f}, c_{\alpha \vec{k}}^{\dagger}\right]=\sum_{\alpha^{\prime}}\left[h_{\alpha \alpha^{\prime}}^{*}(\vec{k}) c_{\alpha^{\prime} \vec{k}}^{\dagger}+\Delta_{\alpha \alpha^{\prime}}^{*}(\vec{k}) c_{\alpha^{\prime}-\vec{k}}\right]
$$

Substituindo a transformação (2.35) em (2.47) chegamos a

$$
\begin{aligned}
{\left[K_{e f}, c_{\alpha \vec{k}}\right] } & =-\sum_{\alpha^{\prime} \gamma}\left(h_{\alpha \alpha^{\prime}}(\vec{k}) u_{\alpha^{\prime} \gamma}(\vec{k})+\Delta_{\alpha \alpha^{\prime}}(\vec{k}) v_{\alpha^{\prime} \gamma}(\vec{k})\right) b_{\gamma \vec{k}}+ \\
& +\left(h_{\alpha \alpha^{\prime}}(\vec{k}) v_{\alpha^{\prime} \gamma}^{*}(\vec{k})+\Delta_{\alpha \alpha^{\prime}}(\vec{k}) u_{\alpha^{\prime} \gamma}^{*}(\vec{k})\right) b_{\gamma-\vec{k}}^{\dagger}
\end{aligned}
$$

e, do mesmo modo, a

$$
\begin{aligned}
{\left[K_{e f}, c_{\alpha \vec{k}}^{\dagger}\right] } & =\sum_{\alpha^{\prime} \gamma}\left(h_{\alpha \alpha^{\prime}}^{*}(\vec{k}) u_{\alpha^{\prime} \gamma}^{*}(\vec{k})+\Delta_{\alpha \alpha^{\prime}}^{*}(\vec{k}) v_{\alpha^{\prime} \gamma}^{*}(\vec{k})\right) b_{\gamma \vec{k}}^{\dagger}+ \\
& +\left(h_{\alpha \alpha^{\prime}}^{*}(\vec{k}) v_{\alpha^{\prime} \gamma}(\vec{k})+\Delta_{\alpha \alpha^{\prime}}^{*}(\vec{k}) u_{\alpha^{\prime} \gamma}(\vec{k})\right) b_{\gamma-\vec{k}}
\end{aligned}
$$

Relacionando (2.48) e (2.49) com (2.41) e (2.44) respectivamente, temos a seguinte expressão

$$
\begin{aligned}
\sum_{\gamma} E_{\gamma}(\vec{k})\left[u_{\alpha \gamma}(\vec{k}) b_{\gamma \vec{k}}-v_{\alpha \gamma}^{*}(\vec{k}) b_{\gamma-\vec{k}}^{\dagger}\right] & =\sum_{\alpha^{\prime} \gamma}\left(h_{\alpha \alpha^{\prime}}(\vec{k}) u_{\alpha^{\prime} \gamma}(\vec{k})+\Delta_{\alpha \alpha^{\prime}}(\vec{k}) v_{\alpha^{\prime} \gamma}(\vec{k})\right) b_{\gamma \vec{k}}+ \\
& +\left(h_{\alpha \alpha^{\prime}}(\vec{k}) v_{\alpha^{\prime} \gamma}^{*}(\vec{k})+\Delta_{\alpha \alpha^{\prime}}(\vec{k}) u_{\alpha^{\prime} \gamma}^{*}(\vec{k})\right) b_{\gamma-\vec{k}}^{\dagger}, \quad(2.5
\end{aligned}
$$

e seu complexo conjugado. 
Como os operadores transformados, $b_{\alpha \vec{k}}^{\dagger}$ e $b_{\alpha \vec{k}}$, são independentes entre si, podemos igualar os coeficientes dos operadores de quasi-partícula em (2.50), e dessa forma obtemos as equações de Hartree-Fock-Bogoliubov do sistema

$$
E_{\gamma}(\vec{k}) u_{\alpha \gamma}(\vec{k})=\sum_{\alpha^{\prime}}\left(h_{\alpha \alpha^{\prime}}(\vec{k}) u_{\alpha^{\prime} \gamma}(\vec{k})+\Delta_{\alpha \alpha^{\prime}}(\vec{k}) v_{\alpha^{\prime} \gamma}(\vec{k})\right)
$$

$\mathrm{e}$

$$
-E_{\gamma}(\vec{k}) v_{\alpha \gamma}(\vec{k})=\sum_{\alpha^{\prime}}\left(h_{\alpha \alpha^{\prime}}^{*}(\vec{k}) v_{\alpha^{\prime} \gamma}(\vec{k})+\Delta_{\alpha \alpha^{\prime}}^{*}(\vec{k}) u_{\alpha^{\prime} \gamma}(\vec{k})\right)
$$

Uma maneira de reescrever (2.51) e (2.52) é usar uma forma matricial

$$
\left(\begin{array}{cccc}
h_{11}(\vec{k}) & h_{12}(\vec{k}) & \Delta_{11}(\vec{k}) & \Delta_{12}(\vec{k}) \\
h_{12}(\vec{k}) & h_{22}(\vec{k}) & \Delta_{12}(\vec{k}) & \Delta_{22}(\vec{k}) \\
\Delta_{11}^{*}(\vec{k}) & \Delta_{12}^{*}(\vec{k}) & h_{11}^{*}(\vec{k}) & h_{12}^{*}(\vec{k}) \\
\Delta_{12}^{*}(\vec{k}) & \Delta_{22}^{*}(\vec{k}) & h_{12}^{*}(\vec{k}) & h_{22}^{*}(\vec{k})
\end{array}\right)\left(\begin{array}{c}
u_{1 \gamma}(\vec{k}) \\
u_{2 \gamma}(\vec{k}) \\
v_{1 \gamma}(\vec{k}) \\
v_{2 \gamma}(\vec{k})
\end{array}\right)=E_{\gamma}(\vec{k})\left(\begin{array}{cccc}
1 & 0 & 0 & 0 \\
0 & 1 & 0 & 0 \\
0 & 0 & -1 & 0 \\
0 & 0 & 0 & -1
\end{array}\right)\left(\begin{array}{c}
u_{1 \gamma}(\vec{k}) \\
u_{2 \gamma}(\vec{k}) \\
v_{1 \gamma}(\vec{k}) \\
v_{2 \gamma}(\vec{k})
\end{array}\right) .
$$

As equações de Hartree-Fock-Bogoliubov, (2.53), equivalem a uma equação secular de uma matriz não hermiteana. Para mostrar essa propriedade basta reescrever (2.53) como

$$
\eta \mathcal{H}(\vec{k}) X_{\gamma}(\vec{k})=E_{\gamma}(\vec{k}) X_{\gamma}(\vec{k})
$$

onde $\eta=\left(\begin{array}{cc}I & 0 \\ 0 & -I\end{array}\right)$ e $\mathcal{H}(\vec{k})=\left(\begin{array}{cc}h(\vec{k}) & \Delta(\vec{k}) \\ \Delta^{*}(\vec{k}) & h^{*}(\vec{k})\end{array}\right)$, ou seja, $\eta \mathcal{H}(\vec{k})=\left(\begin{array}{cc}h(\vec{k}) & \Delta(\vec{k}) \\ -\Delta^{*}(\vec{k}) & -h^{*}(\vec{k})\end{array}\right)$ é a matriz não hermiteana a ser diagonalizada.

Os autovalores reais e positivos de (2.54) são os dois ramos de excitação coletiva do

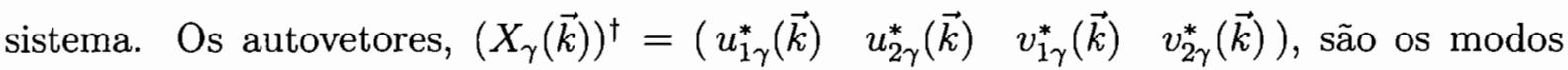
normais e são normalizados com a métrica bosônica, $\left(X_{\gamma}(\vec{k})\right)^{\dagger} \eta X_{\gamma}(\vec{k})=1$.

A equação secular (2.54) não é linear e pode ser calculada de forma auto-consistente, ou seja, a matriz a ser diagonalizada depende dos autovalores, autovetores e da solução de Gross-Pitaeviskii a serem determinados.

\subsection{Equações de Gross-Pitaevskii dependente da tem- peratura}

O funcional, $\phi$, descrito na equação (2.23), depende do operador densidade, (2.21), e das funções de onda dos condensados, $z_{\alpha}$. Como vimos, a minimização desse funcional com respeito às médias térmicas dos produtos bilineares dos operadores deslocados leva 
às equações de Hartree-Fock-Bogoliubov para as excitações coletivas. Por sua vez, a minimização com respeito às funções de onda dos condensados levam às equações de Gross-Pitaevskii.

Assim, ao minimizarmos o funcional em relação às funções de onda do condensado, chegamos a:

$$
\frac{\partial \operatorname{Tr} D(H-\mu N)}{\partial z_{\alpha}^{*}}=\frac{\partial \operatorname{Tr} D(H-\mu N)}{\partial z_{\alpha}}=0
$$

pois o único termo dependente das funções de onda dos condensados, $z_{\alpha}$, é a média térmica do hamiltoniano grão-canônico, $H-\mu N$. Dessa forma, as equações de Gross-Pitaevskii para condensados com duas componentes são dadas por:

$$
\begin{aligned}
& \left(\frac{1}{\Omega} \sum_{\beta} \tilde{V}_{\alpha \beta}(0)\left(\left|z_{\beta}\right|^{2}+\sum_{\vec{k}}\left\langle c_{\beta \vec{k}}^{\dagger} c_{\beta \vec{k}}\right\rangle\right)-\mu\right) z_{\alpha}+ \\
+ & \sum_{\beta}\left(\Lambda_{\alpha \beta}+\frac{1}{\Omega} \sum_{\vec{k}} \tilde{V}_{\alpha \beta}(\vec{k})\left\langle c_{\beta \vec{k}}^{\dagger} c_{\alpha \vec{k}}\right\rangle\right) z_{\beta}+ \\
+ & \frac{1}{\Omega} \sum_{\beta} \sum_{\vec{k}} \tilde{V}_{\alpha \beta}(\vec{k})\left\langle c_{\alpha \vec{k}} c_{\beta-\vec{k}}\right\rangle z_{\beta}^{*}=0 .
\end{aligned}
$$

As quatro equações deduzidas de (2.56) são as equações de Gross-Pitaevskii estendidas para o caso em que o sistema tenha duas componentes. Essas equações dependem das quatro incógnitas, que são as funções de onda dos condensados, $z_{\alpha}$, do potencial químico, $\mu$, das depleções, $\left\langle c_{\beta \vec{k}}^{\dagger} c_{\alpha \vec{k}}\right\rangle$, e das densidades de pares, $\left\langle c_{\beta \vec{k}} c_{\alpha-\vec{k}}\right\rangle$.

As incógnitas devido aos átomos fora do condensado são determinadas pelas equações de Hartree-Fock-Bogoliubov (2.53). Com isso, o sistema de cinco incógnitas necessita de somente mais uma equação para ficar determinado e essa equação vem do número de partículas no sistema dado por:

$$
N=\sum_{\alpha}\left(\left|z_{\alpha}\right|^{2}+\sum_{\vec{k}}\left\langle c_{\alpha \vec{k}}^{\dagger} c_{\alpha \vec{k}}\right\rangle\right)
$$

A equação de número (2.57), as quatro equações de Gross-Pitaevskii (2.56) e as equações de Hartree-Fock-Bogoliubov (2.53) determinam as funções de onda dos condensados, o espectro e os modos de excitação coletiva.

Antes de prosseguir na discussão de como resolver as equações de Gross-Pitaevskii e de Hartree-Fock-Bogoliubov, investigamos as conseqüências da conservação de número total de partículas do sistema nas propriedades dessas equações. 


\subsection{Invariância de calibre do sistema}

Em geral, dada uma transformação, se o hamiltoniano for invariante por essa transformação dizemos que há uma simetria no sistema referente a ela e como conseqüência vemos que há uma constante de movimento associada a essa simetria.

Uma simetria a ser considerada no nosso caso é a conservação de número. Sabemos que o hamiltoniano (2.1) comuta com o operador número, ou seja, o número total de partículas se conserva. Assim, para que a teoria de Hartree-Fock-Bogoliubov fique consistente com essa simetria, esperamos a existência de uma transformação nos parâmetros variacionais que torne as equações de Gross-Pitaeviskii e as equações de Hartree-Fock-Bogoliubov invariantes.

A transformação associada a simetria de número é a transformação de calibre, ou seja, a multiplicação dos operadores de criação e de aniquilação por uma fase,

$$
\left\{\begin{array}{l}
a_{\alpha \vec{k}} \rightarrow e^{-i \theta} a_{\alpha \vec{k}} \\
a_{\alpha \vec{k}}^{\dagger} \rightarrow e^{i \theta} a_{\alpha \vec{k}}^{\dagger}
\end{array} .\right.
$$

É fácil verificar que o hamiltoniano (2.1) permanece o mesmo por essa transformação. Nas funções de onda do condensado e nos operadores deslocados, a transformação (2.58) age como

$$
\left\{\begin{array}{l}
z_{\alpha} \rightarrow e^{-i \theta} z_{\alpha} \\
c_{\alpha \vec{k}} \rightarrow e^{-i \theta} c_{\alpha \vec{k}} \\
c_{\alpha \vec{k}}^{\dagger} \rightarrow e^{i \theta} c_{\alpha \vec{k}}^{\dagger}
\end{array}\right.
$$

o que implica na seguinte transformação dos parâmetros de Bogoliubov,

$$
\left\{\begin{array}{l}
u_{\alpha \beta}(\vec{k}) \rightarrow e^{-i \theta} u_{\alpha \beta}(\vec{k}) \\
v_{\alpha \beta}^{*}(\vec{k}) \rightarrow e^{-i \theta} v_{\alpha \beta}^{*}(\vec{k})
\end{array} .\right.
$$

A transformação (2.58) torna as equações de Gross-Pitaeviskii invariante pois vemos, por inspeção, que o fator $e^{i \theta}$ fatora ao fazer a transformação dos parâmetros nas equações de Gross-Pitaevskii (2.56).

A transformação (2.59) faz com que as expressões dos parâmetros variacionais do hamiltoniano efetivo sofram a seguinte transformação

$$
\left\{\begin{array}{l}
h_{\alpha \beta}(\vec{k}) \rightarrow h_{\alpha \beta}(\vec{k}) \\
\Delta_{\alpha \beta}(\vec{k}) \rightarrow e^{-i 2 \theta} \Delta_{\alpha \beta}(\vec{k})
\end{array}\right.
$$


Para mostrarmos a invariância das equações de Hartree-Fock-Bogoliubov (2.53), aplicamos a transformação (2.59) nelas e vemos que

$$
\left(\begin{array}{cc}
h(\vec{k}) & e^{-i 2 \theta} \Delta(\vec{k}) \\
e^{i 2 \theta} \Delta^{*}(\vec{k}) & h^{*}(\vec{k})
\end{array}\right)\left(\begin{array}{c}
e^{-i \theta} u_{\gamma}(\vec{k}) \\
e^{i \theta} v_{\gamma}(\vec{k})
\end{array}\right)=E_{\gamma}(\vec{k})\left(\begin{array}{cc}
1 & 0 \\
0 & -1
\end{array}\right)\left(\begin{array}{c}
e^{-i \theta} u_{\gamma}(\vec{k}) \\
e^{i \theta} v_{\gamma}(\vec{k})
\end{array}\right),
$$

onde $u_{\gamma}(\vec{k})=\left(\begin{array}{c}u_{1 \gamma}(\vec{k}) \\ u_{2 \gamma}(\vec{k})\end{array}\right)$ e $v_{\gamma}(\vec{k})=\left(\begin{array}{c}v_{1 \gamma}(\vec{k}) \\ v_{2 \gamma}(\vec{k})\end{array}\right)$. Podemos reescrever (2.62) como

$$
\left(\begin{array}{cc}
e^{-i \theta} h(\vec{k}) & e^{-i \theta} \Delta(\vec{k}) \\
e^{i \theta} \Delta^{*}(\vec{k}) & e^{i \theta} h^{*}(\vec{k})
\end{array}\right)\left(\begin{array}{l}
u_{\gamma}(\vec{k}) \\
v_{\gamma}(\vec{k})
\end{array}\right)=E_{\gamma}(\vec{k})\left(\begin{array}{cc}
e^{-i \theta} & 0 \\
0 & -e^{i \theta}
\end{array}\right)\left(\begin{array}{l}
u_{\gamma}(\vec{k}) \\
v_{\gamma}(\vec{k})
\end{array}\right)
$$

e com mais uma manobra algébrica chegamos a

$$
\left(\begin{array}{cc}
e^{-i \theta} & 0 \\
0 & e^{i \theta}
\end{array}\right)\left(\begin{array}{cc}
h(\vec{k}) & \Delta(\vec{k}) \\
\Delta^{*}(\vec{k}) & h^{*}(\vec{k})
\end{array}\right)\left(\begin{array}{l}
u_{\gamma}(\vec{k}) \\
u_{\gamma}(\vec{k})
\end{array}\right)=E_{\gamma}(\vec{k})\left(\begin{array}{cc}
e^{-i \theta} & 0 \\
0 & e^{i \theta}
\end{array}\right)\left(\begin{array}{cc}
1 & 0 \\
0 & -1
\end{array}\right)\left(\begin{array}{l}
u_{\gamma}(\vec{k}) \\
v_{\gamma}(\vec{k})
\end{array}\right)
$$

que nos conduz a invariância das equações de Hartree-Fock-Bogoliubov.

\subsubsection{Simplificações nas equações de Gross-Pitaevskii}

Na secção anterior mostramos que as equações de Gross-Pitaevskii e Hartree-FockBogoliubov são invariantes pela transformação de calibre (2.58). Isto permite assumir que uma das funções de onda do condensado seja real. Assim podemos escolher $z_{1}$ tal que seja real e positiva, isto é, $z_{1}=z_{1}^{*}=\sqrt{N_{01}}$, com $\theta_{1}=0$. Além disso, procuramos soluções das equações de Gross-Pitaevskii tais que as funções de onda dos condensados sejam reais, $z_{2}=\sqrt{N_{02}} e^{-i \theta_{2}} \operatorname{com} \theta_{2}=0$ ou $\theta_{2}=\pi$. A conseqüência dessa restrição é que agora temos duas classes de estados de equilíbrio, uma onde as funções de onda dos condensados têm a mesma fase, $\theta_{12}=\theta_{2}-\theta_{1}=0$ (modo 0$)$, e outra onde têm fases opostas, $\theta_{12}=\theta_{2}-\theta_{1}=\pi$ (modo $\pi$ ). Impondo essas condições nas equações (2.56) podemos escrevê-las como

$$
\begin{aligned}
\mu & =\frac{1}{\Omega} \sum_{\beta} \tilde{V}_{\alpha \beta}(0)\left(N_{0 \beta}+\sum_{\vec{k}}\left\langle c_{\beta \vec{k}}^{\dagger} c_{\beta \vec{k}}\right\rangle\right)+ \\
& +\sum_{\beta} \cos \theta_{\alpha \beta} \sqrt{\frac{N_{0 \beta}}{N_{0 \alpha}}}\left(\Lambda_{\alpha \beta}+\frac{1}{\Omega} \sum_{\vec{k}} \tilde{V}_{\alpha \beta}(\vec{k})\left[\left\langle c_{\beta \vec{k}}^{\dagger} c_{\alpha \vec{k}}\right\rangle+\left\langle c_{\alpha \vec{k}} c_{\beta-\vec{k}}\right\rangle\right]\right)
\end{aligned}
$$

onde $\theta_{\alpha \beta}=\theta_{\beta}-\theta_{\alpha}$ e $\cos \theta_{\alpha \beta}= \pm 1$.

Dessa expressão vemos, imediatamente, que uma condição suficiente para que as funções de onda, $z_{\alpha}$, sejam reais é impor, por sua vez, que as médias térmicas do produto bilinear dos operadores deslocados, $\left\langle c_{\beta \vec{k}}^{\dagger} c_{\alpha \vec{k}}\right\rangle$ e $\left\langle c_{\alpha \vec{k}} c_{\beta-\vec{k}}\right\rangle$ sejam reais. Essas médias 
térmicas estão relacionadas com os autovalores e os autovetores das equações de HartreeFock-Bogoliubov através das expressões

$$
\left\langle c_{\alpha \vec{k}} c_{\beta-\vec{k}}\right\rangle=\sum_{\gamma}\left[\left(\frac{1}{2}+\nu_{\gamma}(\vec{k})\right)\left(u_{\alpha \gamma}(\vec{k}) v_{\beta \gamma}^{*}(\vec{k})+v_{\alpha \gamma}^{*}(\vec{k}) u_{\beta \gamma}(\vec{k})\right)\right],
$$

$\mathrm{e}$

$$
\left\langle c_{\alpha \vec{k}}^{\dagger} c_{\beta \vec{k}}\right\rangle=\sum_{\gamma}\left[\nu_{\gamma}(\vec{k}) u_{\alpha \gamma}(\vec{k}) u_{\beta \gamma}^{*}(\vec{k})+\left(1+\nu_{\gamma}(\vec{k})\right) v_{\alpha \gamma}(\vec{k}) v_{\beta \gamma}^{*}(\vec{k})\right]
$$

onde $\nu_{\gamma}(\vec{k})=\frac{1}{e^{\beta E_{\gamma}(\vec{k})}-1}$ é o número de ocupação de quasi-partículas no modo $\gamma$ e com momento $\vec{k}$. A demonstração de (2.66) e (2.67) pode ser vista no apêndice A.

Com isso, assumir que essas médias térmicas sejam reais é equivalente a tornar as componentes dos autovetores, $u_{\alpha \beta}(\vec{k})$ e $v_{\alpha \beta}(\vec{k})$, reais. Isto é consistente com as equações de Hartree-Fock-Bogoliubov, equação (2.53), pois nesse caso, vemos das equações (2.31) e (2.33) que $h_{\alpha \beta}(\vec{k})$ e $\Delta_{\alpha \beta}(\vec{k})$ são elementos de matrizes reais e simétricas, conseqüentemente, os parâmetros $u_{\alpha \beta}(\vec{k})$ e $v_{\alpha \beta}(\vec{k})$ podem ser escolhidos reais.

Das equações de Gross-Pitaevskii e da equação de número somos capazes de calcular os parâmetros dos condensados, $N_{0 \alpha}$ e $\mu$. Realmente, igualando as duas expressões para o potencial químico em (2.65) chegamos a

$$
\begin{aligned}
0 & =\Lambda_{11}-\Lambda_{22}+\frac{1}{\Omega} \tilde{V}_{11}(0)\left(N_{01}+\sum_{\vec{k}}\left\langle c_{1 \bar{k}}^{\dagger} c_{1 \vec{k}}\right\rangle\right)-\frac{1}{\Omega} \tilde{V}_{22}(0)\left(N_{02}+\sum_{\vec{k}}\left\langle c_{2 \vec{k}}^{\dagger} c_{2 \vec{k}}\right\rangle\right)+ \\
& +\frac{1}{\Omega} \tilde{V}_{12}(0)\left(N_{02}+\sum_{\vec{k}}\left\langle c_{2 \vec{k}}^{\dagger} c_{2 \vec{k}}\right\rangle-N_{01}-\sum_{\vec{k}}\left\langle c_{1 \vec{k}}^{\dagger} c_{1 \vec{k}}\right\rangle\right)+ \\
& +\frac{1}{\Omega} \sum_{\vec{k}}\left[\tilde{V}_{11}(\vec{k})\left(\left\langle c_{1 \vec{k}}^{\dagger} c_{1 \vec{k}}\right\rangle+\left\langle c_{1 \vec{k}} c_{1-\vec{k}}\right\rangle\right)-\tilde{V}_{22}(\vec{k})\left(\left\langle c_{2 \vec{k}}^{\dagger} c_{2 \vec{k}}\right\rangle+\left\langle c_{2 \vec{k}} c_{2-\vec{k}}\right\rangle\right)\right]+ \\
& +\left(\sqrt{\frac{N_{02}}{N_{01}}}-\sqrt{\frac{N_{01}}{N_{02}}}\right) \cos \theta_{12}\left[J+\frac{1}{\Omega} \sum_{\vec{k}} \tilde{V}_{12}(\vec{k})\left\langle c_{1 \vec{k}}^{\dagger} c_{2 \vec{k}}\right\rangle+\frac{1}{\Omega} \sum_{\vec{k}} \tilde{V}_{12}(\vec{k})\left\langle c_{1 \vec{k}} c_{2-\vec{k}}\right\rangle\right]
\end{aligned}
$$

com

$$
N=N_{01}+N_{02}+\sum_{\alpha} \sum_{\vec{k}}\left\langle c_{\alpha \vec{k}}^{\dagger} c_{\alpha \vec{k}}\right\rangle
$$

o que determina os valores de $N_{01}$ e $N_{02}$, desde que sejam conhecidos os valores das médias térmicas dos produtos de $c_{\alpha \vec{k}}^{\dagger}$ e $c_{\alpha \vec{k}}$.

Para simplificar as equações de Gross-Pitaevskii, introduzimos a fração relativa de átomos nos dois condensados, $\bar{f}$, como a razão da diferença de número de átomos condensados em cada estado hiperfino pelo número total de átomos condensados

$$
\bar{f}=\frac{N_{01}-N_{02}}{N_{01}+N_{02}}
$$


e a depleção total do sistema, $\chi$, como a razão do número total de átomos fora do condensado de ambas espécies pelo número total de átomos

$$
\chi=\frac{1}{N} \sum_{\vec{k}}\left[\left\langle c_{1 \vec{k}}^{\dagger} c_{1 \vec{k}}\right\rangle+\left\langle c_{2 \vec{k}}^{\dagger} c_{2 \vec{k}}\right\rangle\right] .
$$

Das definições (2.69), (2.70) e (2.71), o número de átomos condensados em cada estado hiperfino é dado em termos dessas quantidades como

$$
\left\{\begin{array}{l}
N_{01}=\frac{N}{2}(1-\chi)(1+\bar{f}) \\
N_{02}=\frac{N}{2}(1-\chi)(1-\bar{f})
\end{array}\right.
$$

$\mathrm{e}$

$$
\sqrt{\frac{N_{02}}{N_{01}}}-\sqrt{\frac{N_{01}}{N_{02}}}=-\frac{2 \bar{f}}{\sqrt{1-\bar{f}^{2}}} .
$$

Com isso podemos reescrever (2.68) como sendo

$$
\begin{aligned}
0 & =\delta+\frac{\rho(1-\chi)}{2}\left(\tilde{V}_{11}(0)-\tilde{V}_{22}(0)\right)+ \\
& +\frac{1}{\Omega} \sum_{\vec{k}}\left[\left(\tilde{V}_{11}(0)+\tilde{V}_{11}(\vec{k})-\tilde{V}_{12}(0)\right)\left\langle c_{1 \vec{k}}^{\dagger} c_{1 \vec{k}}\right\rangle+\tilde{V}_{11}(\vec{k})\left\langle c_{1 \vec{k}} c_{1-\vec{k}}\right\rangle\right]- \\
& -\frac{1}{\Omega} \sum_{\vec{k}}\left[\left(\tilde{V}_{22}(0)+\tilde{V}_{22}(\vec{k})-\tilde{V}_{12}(0)\right)\left\langle c_{2 \vec{k}}^{\dagger} c_{2 \vec{k}}\right\rangle+\tilde{V}_{22}(\vec{k})\left\langle c_{2 \vec{k}} c_{2-\vec{k}}\right\rangle\right]+ \\
& +\frac{\rho(1-\chi)}{2}\left(\tilde{V}_{11}(0)+\tilde{V}_{22}(0)-2 \tilde{V}_{12}(0)\right) \bar{f}- \\
& -\cos \theta_{12}\left[J+\frac{1}{\Omega} \sum_{\vec{k}} \tilde{V}_{12}(\vec{k})\left(\left\langle c_{1 \vec{k}}^{\dagger} c_{2 \vec{k}}\right\rangle+\left\langle c_{1 \vec{k}} c_{2-\vec{k}}\right\rangle\right)\right] \frac{2 \vec{f}}{\sqrt{1-\bar{f}^{2}}}
\end{aligned}
$$

onde $\rho$ é a densidade total do sistema.

Vemos, na equação (2.74), um termo independente de $\bar{f}$, um termo linear em $\bar{f}$ e, por último, um termo linear em $\bar{f} / \sqrt{1-\bar{f}^{2}}$. Para esclarecer o significado físico dessa equação que determina a fração relativa de átomos nos condensados, definimos os coeficientes de cada um dos termos acima.

O termo independente de $\bar{f}$ é a dessintonia efetiva

$$
\begin{aligned}
\delta_{e f} & =\frac{1}{2}\left\{\delta+\frac{\rho(1-\chi)}{2}\left(\tilde{V}_{11}(0)-\tilde{V}_{22}(0)\right)+\right. \\
& +\frac{1}{\Omega} \sum_{\vec{k}}\left[\left(\tilde{V}_{11}(0)+\tilde{V}_{11}(\vec{k})-\tilde{V}_{12}(0)\right)\left\langle c_{1 \vec{k}}^{\dagger} c_{1 \vec{k}}\right\rangle+\tilde{V}_{11}(\vec{k})\left\langle c_{1 \vec{k}} c_{1-\vec{k}}\right\rangle\right]- \\
& \left.-\frac{1}{\Omega} \sum_{\vec{k}}\left[\left(\tilde{V}_{22}(0)+\tilde{V}_{22}(\vec{k})-\tilde{V}_{12}(0)\right)\left\langle c_{2 \vec{k}}^{\dagger} c_{2 \vec{k}}\right\rangle+\tilde{V}_{22}(\vec{k})\left\langle c_{2 \vec{k}} c_{2-\vec{k}}\right\rangle\right]\right\},
\end{aligned}
$$


o coeficiente do termo linear em $\bar{f}$ é o parâmetro de interação mútua efetiva

$$
\mathrm{a}_{e f}=\frac{\rho(1-\chi)}{4}\left(\tilde{V}_{11}(0)+\tilde{V}_{22}(0)-2 \tilde{V}_{12}(0)\right)
$$

e, finalmente, o coeficiente do último termo é o coeficiente de Josephson efetivo dado por:

$$
J_{e f}=J+\frac{1}{\Omega} \sum_{\vec{k}} \tilde{V}_{12}(\vec{k})\left\langle c_{1 \vec{k}}^{\dagger} c_{2 \vec{k}}\right\rangle+\frac{1}{\Omega} \sum_{\vec{k}} \tilde{V}_{12}(\vec{k})\left\langle c_{1 \vec{k}} c_{2-\vec{k}}\right\rangle .
$$

Diferentemente da referência [70], a dessintonia efetiva, $\delta_{e f}$, é a dessintonia do sistema em temperatura nula e depleção desprezível mais contribuições que envolvem a interação de átomos condensados e não condensados. Por sua vez, a interação mútua, $a_{e f}$, obtida no nosso caso difere do correspondente em [70] por ser proporcional à densidade do condensado enquanto em [70] é proporcional à densidade do sistema.

O Josephson efetivo difere do definido em [70] por conter termos que envolvem a interação entre átomos condensados e átomos não condensados. Como exemplo para elucidar o significado físico desses termos, vemos que, do mesmo modo que o acoplamento de Josephson é responsável pela transição que troca o estado hiperfino de átomos nos condensados, o segundo termo de (2.77) faz essa transição através da interação entre um átomo no condensado com um não condensado.

Introduzidos esses parâmetros, podemos reescrever a equação (2.74) como

$$
\delta_{e f}+a_{e f} \bar{f}-J_{e f} \cos \theta_{12} \frac{\bar{f}}{\sqrt{1-\bar{f}^{2}}}=0
$$

que se dividirmos por $J_{e f}$ teremos uma expressão dada por

$$
\Delta_{e f}+A_{e f} \bar{f}-\cos \theta_{12} \frac{\bar{f}}{\sqrt{1-\bar{f}^{2}}}=0
$$

onde $\Delta_{e f}=\delta_{e f} / J_{e f}$ e $A_{e f}=a_{e f} / J_{e f}$. Comparando com a obtida em [70] vemos que elas têm a mesma forma diferindo no fato de que, no nosso caso, os parâmetros de controle dependem do estado do sistema. Naturalmente, se desprezarmos as quantidades que dependem dos átomos fora dos condensados na equação (2.79), retornaríamos à equação da referência [70]. 


\subsubsection{As energias das excitações coletivas}

Ao escolhermos que todos os parâmetros das equações de Gross-Pitaevskii sejam reais, vemos, por inspeção, que os parâmetros do hamiltoniano efetivo são reais,

$$
\left\{\begin{array}{l}
h_{\alpha \beta}(\vec{k})=h_{\alpha \beta}^{*}(\vec{k}) \\
\Delta_{\alpha \beta}(\vec{k})=\Delta_{\alpha \beta}^{*}(\vec{k})
\end{array} .\right.
$$

Isso simplifica a equação secular, (2.54), pois agora temos um problema de diagonalização,

$$
\left(\begin{array}{cccc}
h_{11}(\vec{k}) & h_{12}(\vec{k}) & \Delta_{11}(\vec{k}) & \Delta_{12}(\vec{k}) \\
h_{12}(\vec{k}) & h_{22}(\vec{k}) & \Delta_{12}(\vec{k}) & \Delta_{22}(\vec{k}) \\
-\Delta_{11}(\vec{k}) & -\Delta_{12}(\vec{k}) & -h_{11}(\vec{k}) & -h_{12}(\vec{k}) \\
-\Delta_{12}(\vec{k}) & -\Delta_{22}(\vec{k}) & -h_{12}(\vec{k}) & -h_{22}(\vec{k})
\end{array}\right)\left(\begin{array}{c}
u_{1 \gamma}(\vec{k}) \\
u_{2 \gamma}(\vec{k}) \\
v_{1 \gamma}(\vec{k}) \\
v_{2 \gamma}(\vec{k})
\end{array}\right)=E_{\gamma}(\vec{k})\left(\begin{array}{c}
u_{1 \gamma}(\vec{k}) \\
u_{2 \gamma}(\vec{k}) \\
v_{1 \gamma}(\vec{k}) \\
v_{2 \gamma}(\vec{k})
\end{array}\right),
$$

onde a matriz a ser diagonalizada é real.

Como queremos estados de equilíbrio estáveis, devemos considerar somente as energias de excitação do sistema que forem reais e positivas. Essa condição implica que a matriz, $\mathcal{H}(\vec{k})$, seja sempre definida semi-positiva, como demonstrado na referência [71], Uma conseqüência dessa propriedade é que os autovetores podem ser escolhidos reais. Desse fato, concluímos que as soluções de Hartree-Fock-Bogoliubov são consistentes com as considerações feitas para as soluções da equação de Gross-Pitaevskii.

Ao diagonalizar (2.81) chegamos à equação de quarto grau nos autovalores dada por:

$$
E_{\gamma}(\vec{k})^{4}-\tilde{\epsilon}(\vec{k})^{2} E_{\gamma}(\vec{k})^{2}+\tilde{\Gamma}(\vec{k})^{4}=0
$$

onde a solução são os dois ramos de energia

$$
\left\{\begin{array}{l}
E_{-}(\vec{k})=\frac{1}{\sqrt{2}} \sqrt{\tilde{\epsilon}(\vec{k})^{2}-\sqrt{\tilde{\epsilon}(\vec{k})^{4}-4 \tilde{\Gamma}(\vec{k})^{4}}} \\
E_{+}(\vec{k})=\frac{1}{\sqrt{2}} \sqrt{\tilde{\epsilon}(\vec{k})^{2}+\sqrt{\tilde{\epsilon}(\vec{k})^{4}-4 \tilde{\Gamma}(\vec{k})^{4}}}
\end{array}\right.
$$

com

$$
\tilde{\epsilon}(\vec{k})^{2}=\left(h_{11}^{2}(\vec{k})+h_{22}^{2}(\vec{k})+2 h_{12}^{2}(\vec{k})\right)-\left(\Delta_{11}^{2}(\vec{k})+\Delta_{22}^{2}(\vec{k})+2 \Delta_{12}^{2}(\vec{k})\right)
$$

$\mathrm{e}$

$$
\begin{aligned}
\tilde{\Gamma}(\vec{k})^{4} & =\left(\Delta_{12}^{2}(\vec{k})-\Delta_{11}(\vec{k}) \Delta_{22}(\vec{k})\right)^{2}+\left(h_{12}^{2}(\vec{k})-h_{11}(\vec{k}) h_{22}(\vec{k})\right)^{2}- \\
& -\left(\Delta_{12}(\vec{k}) h_{12}(\vec{k})-h_{11}(\vec{k}) \Delta_{22}(\vec{k})\right)^{2}-\left(\Delta_{12}(\vec{k}) h_{12}(\vec{k})-h_{22}(\vec{k}) \Delta_{11}(\vec{k})\right)^{2}- \\
& -2\left(h_{11}(\vec{k}) \Delta_{12}(\vec{k})-h_{12}(\vec{k}) \Delta_{11}(\vec{k})\right)\left(h_{22}(\vec{k}) \Delta_{12}(\vec{k})-h_{12}(\vec{k}) \Delta_{22}(\vec{k})\right)
\end{aligned}
$$


Apesar de ser possível encontrar expressões analíticas para os autovetores de (2.81), não fizemos pois sua forma explícita é complicada e desnecessária para a compreensão das propriedades físicas do sistema.

No limite de momento nulo esperamos, pelo teorema de Hugenholtz-Pines [60], que o ramo de menor energia se anule. No entanto, a presença da densidade anômala, $\left\langle c_{\alpha \vec{k}} c_{\beta-\vec{k}}\right\rangle$, que corresponde a correlação de pares de átomos fora dos condensados com momentos opostos, introduz uma lacuna nas energias de excitação no limite de momento nulo.

O mesmo acontece em condensados com uma componente. Nesse caso, o problema da existência da lacuna é contornado desprezando os termos que envolvem a densidade anômala. Essa aproximação ad hoc, conhecida como a aproximação de Popov, tem uma análoga no caso de condensados de duas componentes acopladas como demonstraremos adiante.

\subsection{Aproximação de Popov}

Como dissemos, uma maneira ad hoc de corrigirmos a teoria, de forma que um dos ramos do espectro de excitação não tenha lacuna, é desprezar a densidade anômala do sistema, $\left\langle c_{\alpha \vec{k}} c_{\beta-\vec{k}}\right\rangle \equiv 0$. Ao fazermos isso, vemos que os termos de emparelhamento dependem somente das funções de onda dos condensados,

$$
\Delta_{\alpha \beta}(\vec{k})=\frac{1}{\Omega} \tilde{V}_{\alpha \beta}(\vec{k}) z_{\alpha} z_{\beta}
$$

o que mostraremos implicar em

$$
\lim _{\vec{k} \rightarrow 0} E_{-}(\vec{k})=0
$$

evitando, com isso, a lacuna no espectro de menor energia de excitação do sistema.

Provamos a afirmação (2.87) mostrando que se desprezarmos as densidades anômalas, $\left\langle c_{\alpha \vec{k}} c_{\beta-\vec{k}}\right\rangle$ e $\left\langle c_{\alpha \vec{k}}^{\dagger} c_{\beta-\vec{k}}^{\dagger}\right\rangle$, nas equações de Gross-Pitaevskii (2.56) e de Hartree-Fock-Bogoliubov (2.51) e (2.52), as equações de Hartree-Fock-Bogoliubov têm uma solução de energia zero quando o momento da excitação é nulo, $\vec{k}=0$.

Realmente vemos que, desprezando a densidade anômala e no limite de $\vec{k}=0$, as equações de Gross-Pitaevskii (2.56) são dadas por

$$
\left(\sum_{\beta} \tilde{V}_{\alpha \beta}(0) \frac{N_{\beta}}{\Omega}-\mu\right) z_{\alpha}+\sum_{\beta}\left(\Lambda_{\alpha \beta}+\frac{1}{\Omega} \sum_{\vec{k}} \tilde{V}_{\alpha \beta}(\vec{k})\left\langle c_{\beta \vec{k}}^{\dagger} c_{\alpha \vec{k}}\right\rangle\right) z_{\beta}=0
$$


onde $N_{\beta}$ é o número total de partículas no $\beta$-ésimo estado hiperfino. E as equações de Hartree-Fock-Bogoliubov são dadas por

$$
\begin{aligned}
E_{\gamma}(0) u_{\alpha \gamma}(0) & =\left(\sum_{\beta} \tilde{V}_{\alpha \beta}(0) \frac{N_{\beta}}{\Omega}-\mu\right) u_{\alpha \gamma}(0)+ \\
& +\sum_{\beta}\left(\Lambda_{\alpha \beta}+\tilde{V}_{\alpha \beta}(0) \frac{z_{\beta}^{*} z_{\alpha}}{\Omega}+\frac{1}{\Omega} \sum_{\vec{k}} \tilde{V}_{\alpha \beta}(\vec{k})\left\langle c_{\beta \vec{k}}^{\dagger} c_{\alpha \vec{k}}\right\rangle\right) u_{\beta \gamma}(0)+ \\
& +\sum_{\beta} \tilde{V}_{\alpha \beta}(0) \frac{z_{\alpha} z_{\beta}}{\Omega} v_{\beta \gamma}(0)
\end{aligned}
$$

$\mathrm{e}$

$$
\begin{aligned}
-E_{\gamma}(0) v_{\alpha \gamma}(0) & =\left(\sum_{\beta} \tilde{V}_{\alpha \beta}(0) \frac{N_{\beta}}{\Omega}-\mu\right) v_{\alpha \gamma}(0)+ \\
& +\sum_{\beta}\left(\Lambda_{\alpha \beta}+\tilde{V}_{\alpha \beta}(0) \frac{z_{\alpha}^{*} z_{\beta}}{\Omega}+\frac{1}{\Omega} \sum_{\vec{k}} \tilde{V}_{\alpha \beta}(\vec{k})\left\langle c_{\alpha \vec{k}}^{\dagger} c_{\beta \vec{k}}\right\rangle\right) v_{\beta \gamma}(0)+ \\
& +\sum_{\beta} \tilde{V}_{\alpha \beta}(0) \frac{z_{\alpha}^{*} z_{\beta}^{*}}{\Omega} u_{\beta \gamma}(0) .
\end{aligned}
$$

Ao identificarmos $u_{\beta \gamma}(0)=z_{\beta}$ e $v_{\beta \gamma}(0)=-z_{\beta}^{*}$ e considerando a energia nula, $E_{\gamma}(0)=$ 0 , temos uma solução para as equações de Hartree-Fock-Bogoliubov na aproximação de Popov com energia nula, pois a equação (2.89) se reduz a equação de Gross-Pitaevskii (2.88) e a equação (2.90) a seu complexo conjugado. Para completar a demonstração, só nos resta identificar o modo de energia nula no limite de momento nulo como sendo o modo (-). Como consideramos somente energias de excitação reais e positivas, sabemos que a energia do modo $(+)$ é maior ou igual ao modo $(-), E_{+}(\vec{k}) \geq E_{-}(\vec{k})$, e, portanto, o ramo que se anula na aproximação de Popov é o do modo (-).

Salientamos que a associação dos autovetores no limite de $\vec{k} \rightarrow 0$ aos modos de Goldstone, modos que se degeneram com o condensado, só é possível se fizermos a aproximação ad hoc de Popov. Apesar de não conseguirmos buscar uma justificativa razoável para essa aproximação, vamos usá-la daqui em diante pois ela considera a interação de pares no condensado e, diferente da teoria de Hartree-Fock-Bogoliubov, não contem a indesejável lacuna no espectro de energia.

Nesse ponto é apropriado especificar o potencial de interação entre os átomos. No caso de um gás diluído, ele pode ser aproximado por uma função finita, independente do momento, parametrizada pelo comprimento de espalhamento de onda $s, a_{\alpha \beta}$, e dado por

$$
\tilde{V}_{\alpha \beta}(\vec{k})=\frac{4 \pi \hbar^{2} a_{\alpha \beta}}{m} \equiv \lambda_{\alpha \beta}
$$


onde uma discussão sobre a validade dessa aproximação pode ser encontrada na referência [73] e usaremos essa aproximação daqui por diante.

\subsubsection{A equação de Gross-Pitaevskii na aproximação de Popov}

Diante de tais aproximações, a equação de Gross-Pitaevskii é escrita em função da densidade de partículas de cada espécie presente nos condensados, $\rho_{01}=N_{01} / \Omega$ e $\rho_{02}=$ $N_{02} / \Omega$, e em função da densidade de partículas de cada espécie fora do condensado, denominadas como depleções do sistema e definidas como

$$
d_{\beta \alpha}=\frac{1}{\Omega} \sum_{\vec{k}}\left\langle c_{\alpha \vec{k}}^{\dagger} c_{\beta \vec{k}}\right\rangle .
$$

No limite termodinâmico ${ }^{2}$, a depleção é determinada pela integral

$$
d_{\beta \alpha}=\frac{1}{2 \pi^{2}} \int_{0}^{\infty}\left\langle c_{\alpha \vec{k}}^{\dagger} c_{\beta \vec{k}}\right\rangle k^{2} d k
$$

onde a média térmica, $\left\langle c_{\alpha \vec{k}}^{\dagger} c_{\beta \vec{k}}\right\rangle$, depende do módulo de $\vec{k}$ e, de (2.67), pode ser escrita como

$$
\left\langle c_{\alpha \vec{k}}^{\dagger} c_{\beta \vec{k}}\right\rangle=\sum_{\gamma}\left[\nu_{\gamma}(k) u_{\alpha \gamma}(k) u_{\beta \gamma}(k)+\left(1+\nu_{\gamma}(k)\right) v_{\alpha \gamma}(k) v_{\beta \gamma}(k)\right]
$$

Com as densidades e as depleções definidas no limite termodinâmico, vemos que a dessintonia efetiva em (2.78) é dada por

$$
2 \delta_{e f}=\delta+\frac{\rho(1+\chi)}{2}\left(\lambda_{11}-\lambda_{22}\right)+\left(\lambda_{11}+\lambda_{22}-\lambda_{12}\right)\left(d_{11}-d_{22}\right)
$$

a interação mútua é reescrita como

$$
\mathrm{a}_{e f}=\frac{\rho(1-\chi)}{4}\left(\lambda_{11}+\lambda_{22}-2 \lambda_{12}\right)
$$

e o coeficiente de Josephson efetivo é

$$
J_{e f}=J+\lambda_{12} d_{12} .
$$

\footnotetext{
${ }^{2}$ Ao aplicar o limite termodinâmico $(N \rightarrow \infty, \Omega \rightarrow \infty$ e $N / \Omega \rightarrow \rho \neq 0)$ a somatória nos momentos transforma-se em $\sum_{\vec{k}} \rightarrow \frac{\Omega}{(2 \pi)^{3}} \int k^{2} \sin \theta_{k} d k d \theta_{k} d \varphi_{k}$.
} 


\subsubsection{A lacuna e a velocidade do som na aproximação de Popov}

As aproximações feitas para esse modelo faz com que os parâmetros do hamiltoniano efetivo possam ser escritos, também, em função da densidade do condensado e da depleção. Dessa forma, os parâmetros são dados por

$$
\begin{aligned}
h_{\alpha \beta}(\vec{k}) & =\left(\frac{\hbar^{2} k^{2}}{2 m}-\mu+\sum_{\alpha^{\prime}} \lambda_{\alpha \alpha^{\prime}}\left(\rho_{0 \alpha^{\prime}}+d_{\alpha \alpha^{\prime}}\right)\right) \delta_{\alpha, \beta}+ \\
& +\Lambda_{\alpha \beta}+\lambda_{\alpha \beta}\left[\cos \theta_{\alpha \beta} \sqrt{\rho_{0 \alpha} \rho_{0 \beta}}+d_{\alpha \beta}\right]
\end{aligned}
$$

que ao substituir o potencial químico,

$$
\mu=\sum_{\beta}\left[\lambda_{\alpha \beta}\left(\rho_{0 \beta}+d_{\beta \beta}\right)+\cos \theta_{\alpha \beta} \sqrt{\frac{\rho_{0 \beta}}{\rho_{0 \alpha}}}\left(\Lambda_{\alpha \beta}+\lambda_{\alpha \beta} d_{\alpha \beta}\right)\right],
$$

determinado pelas equações de Gross-Pitaevskii em (2.98), temos

$$
\left\{\begin{array}{l}
h_{11}(\vec{k})=\frac{\hbar^{2} k^{2}}{2 m}+\lambda_{11} \rho_{01}-\cos \theta_{12} \sqrt{\frac{\rho_{02}}{\rho_{01}}}\left(J+\lambda_{12} d_{12}\right) \\
h_{22}(\vec{k})=\frac{\hbar^{2} k^{2}}{2 m}+\lambda_{22} \rho_{02}-\cos \theta_{12} \sqrt{\frac{\rho_{01}}{\rho_{02}}}\left(J+\lambda_{12} d_{12}\right) \\
h_{12}=J+\lambda_{12} d_{12}+\lambda_{12} \cos \theta_{12} \sqrt{\rho_{01} \rho_{02}}
\end{array}\right.
$$

e os emparelhamentos

$$
\Delta_{\alpha \beta}=\cos \theta_{\alpha \beta} \lambda_{\alpha \beta} \sqrt{\rho_{0 \alpha} \rho_{0 \beta}}
$$

Considerando o modo $\pi$, pois o estado fundamental está nesta classe de soluções [70], e dado as expressões analíticas para os parâmetros (2.100) e (2.101), podemos, pelas equações $(2.83),(2.84)$ e (2.85), achar expressões explícitas para as energias de excitação do sistema.

Assim, de (2.84), vemos que $\tilde{\epsilon}(\vec{k})^{2}$ é um polinômio de segunda ordem em $\epsilon_{k}$,

$$
\tilde{\epsilon}(\vec{k})^{2}=2 \epsilon_{k}^{2}+2 c_{1} \epsilon_{k}+c_{0}
$$

onde

$$
\begin{gathered}
c_{0}=J_{e f}^{2}\left(\sqrt{\frac{\rho_{01}}{\rho_{02}}}+\sqrt{\frac{\rho_{02}}{\rho_{01}}}\right)^{2}+2 J_{e f} \sqrt{\rho_{01} \rho_{02}}\left(\lambda_{11}+\lambda_{22}-2 \lambda_{12}\right) \\
c_{1}=J_{e f}\left(\sqrt{\frac{\rho_{01}}{\rho_{02}}}+\sqrt{\frac{\rho_{02}}{\rho_{01}}}\right)+\lambda_{11} \rho_{01}+\lambda_{22} \rho_{02}
\end{gathered}
$$


$\mathrm{e}$

$$
\epsilon_{k}=\frac{\hbar^{2} k^{2}}{2 m}
$$

é a energia cinética de cada átomo.

Por sua vez, o discriminante das energias de excitação do sistema, $\tilde{\epsilon}(\vec{k})^{4}-4 \tilde{\Gamma}(\vec{k})^{4}$, é, também, um polinômio de segunda ordem em $\epsilon_{k}$,

$$
\tilde{\epsilon}(\vec{k})^{4}-4 \tilde{\Gamma}(\vec{k})^{4}=4 c_{3} \epsilon_{k}^{2}+4 c_{2} \epsilon_{k}+c_{0}^{2}
$$

com

$$
\begin{aligned}
c_{2} & =\left(J_{e f}\left(\sqrt{\frac{\rho_{01}}{\rho_{02}}}+\sqrt{\frac{\rho_{02}}{\rho_{01}}}\right)+\lambda_{11} \rho_{01}+\lambda_{22} \rho_{02}\right) \times \\
& \times\left(J_{e f}^{2}\left(\sqrt{\frac{\rho_{01}}{\rho_{02}}}+\sqrt{\frac{\rho_{02}}{\rho_{01}}}\right)^{2}+2 J_{e f} \sqrt{\rho_{01} \rho_{02}}\left(\lambda_{11}+\lambda_{22}-2 \lambda_{12}\right)\right)- \\
& -J_{\text {ef }}\left(\sqrt{\frac{\rho_{01}}{\rho_{02}}}+\sqrt{\frac{\rho_{02}}{\rho_{01}}}\right)\left(2 J_{\text {ef }} \sqrt{\rho_{01} \rho_{02}}\left(\lambda_{11} \frac{\rho_{01}}{\rho_{02}}+\lambda_{22} \frac{\rho_{02}}{\rho_{01}}+2 \lambda_{12}\right)+4 \rho_{01} \rho_{02}\left(\lambda_{11} \lambda_{22}-\lambda_{12}^{2}\right)\right)
\end{aligned}
$$

$\mathrm{e}$

$$
\begin{aligned}
c_{3} & =J_{\text {ef }}^{2}\left(\sqrt{\frac{\rho_{01}}{\rho_{02}}}+\sqrt{\frac{\rho_{02}}{\rho_{01}}}\right)^{2}+2 J_{e f} \sqrt{\rho_{01} \rho_{02}}\left(\lambda_{11}+\lambda_{22}-2 \lambda_{12}\right)- \\
& -2 J_{e f} \sqrt{\rho_{01} \rho_{02}}\left(\lambda_{11} \frac{\rho_{01}}{\rho_{02}}+\lambda_{22} \frac{\rho_{02}}{\rho_{01}}+2 \lambda_{12}\right)+\left(\lambda_{11} \rho_{01}+\lambda_{22} \rho_{02}\right)^{2}-4 \rho_{01} \rho_{02}\left(\lambda_{11} \lambda_{22}-\lambda_{12}^{2}\right) .
\end{aligned}
$$

Com isso, os dois ramos de energia de excitação são dados por

$$
E_{ \pm}(\vec{k})=\sqrt{\epsilon_{k}^{2}+c_{1} \epsilon_{k}+\frac{c_{0}}{2} \pm \sqrt{c_{3} \epsilon_{k}^{2}+c_{2} \epsilon_{k}+\frac{c_{0}^{2}}{4}}}
$$

No limite de momento nulo, o ramo de energia de excitação do modo $(-), E_{-}(\vec{k})$, tende linearmente a zero, como esperado, e o outro, $E_{+}(\vec{k})$, tende a um valor finito, a lacuna, e dado por

$$
g=E_{+}(0)=\sqrt{c_{0}}
$$

Do ramo do modo (-), podemos extrair a velocidade do som segundo o critério de Landau [74] na qual

$$
v_{s}=\lim _{\vec{k} \rightarrow 0} \frac{\partial E_{-}}{\hbar \partial k}(k)
$$


assim obtemos

$$
v_{s}^{2}=\frac{1}{2 m}\left(c_{1}-\frac{c_{2}}{c_{0}}\right)
$$

ou, mais explicitamente,

$$
v_{s}^{2}=\frac{\left(\rho_{01}+\rho_{02}\right)}{m} \frac{\left(J_{e f}\left(\lambda_{11} \frac{\rho_{01}}{\rho_{02}}+\lambda_{22} \frac{\rho_{02}}{\rho_{01}}+2 \lambda_{12}\right)+2 \sqrt{\rho_{01} \rho_{02}}\left(\lambda_{11} \lambda_{22}-\lambda_{12}^{2}\right)\right)}{J_{e f}\left(\sqrt{\frac{\rho_{01}}{\rho_{02}}}+\sqrt{\frac{\rho_{02}}{\rho_{01}}}\right)^{2}+2 \sqrt{\rho_{01} \rho_{02}}\left(\lambda_{11}+\lambda_{22}-2 \lambda_{12}\right)}
$$

Inspecionando as expressões para as energias de excitação, (2.109), vemos que no limite de momento muito grande, $\vec{k} \rightarrow \infty$, as energias convergem para a energia cinética dos átomos somado a uma constante,

$$
\left\{\begin{array}{l}
E_{-}(\vec{k} \rightarrow \infty) \rightarrow \epsilon_{k}+\frac{c_{1}-\sqrt{c_{3}}}{2} \\
E_{+}(\vec{k} \rightarrow \infty) \rightarrow \epsilon_{k}+\frac{c_{1}+\sqrt{c_{3}}}{2}
\end{array}\right.
$$

A velocidade do som, por ser proporcional a densidade de átomos condensados, $v_{s}^{2} \propto$ $\left(\rho_{01}+\rho_{02}\right)$, tende a zero na temperatura de transição, entretanto o mesmo não acontece para a lacuna pois essa tende a $\frac{2 J_{e f}}{\sqrt{1-\bar{f}^{2}}}$ que não se anula. 


\section{Capítulo 3}

\section{Apresentação e comentários dos resultados numéricos}

\subsection{Método da solução das equações de Gross-Pitaevskii e de Hartree-Fock-Bogoliubov-Popov}

Entendemos o sistema de equações como dois grupos, a equação de Gross-Pitaevskii que determina as densidades dos condensados de cada espécie no estado de equilíbrio, e as equações de Hartree-Fock-Bogoliubov-Popov pelas quais determinamos os modos de excitação coletiva do sistema. Esses dois grupos são dependentes entre si, ou seja, há um acoplamento entre a densidade de partículas no condensado e os modos coletivos do sistema. O acoplamento na equação de Gross-Pitaevskii está na presença das depleções, $d_{\alpha \beta}$, nas expressões que definem os parâmetros de controle do sistema. Já nas equações de Hartree-Fock-Bogoliubov-Popov, ao construirmos os elementos de matriz, vemos que eles dependem da densidade do condensado em ambos estados hiperfinos. Posto dessa forma, vemos que a equação de Gross-Pitaevskii e as equações de Hartree-Fock-Bogoliubov-Popov devem ser resolvidas auto-consistentemente e simultaneamente.

Estamos interessados em resolver a equação de Gross-Pitaevskii e as equações de Hartree-Fock-Bogoliubov-Popov para uma temperatura, $T$, menor que a temperatura de transição na qual o sistema deixa de ter uma população macroscópica no estado com momento nulo. Para isso, dividimos o eixo da temperatura em intervalos iguais e, para cada temperatura, calculamos de maneira auto-consistente as equações de Gross-Pitaevskii e 
de Hartree-Fock-Bogoliubov-Popov.

$\mathrm{Na}$ figura 3.1 esboçamos, em um fluxograma, a rotina usada para calcular as frações de partículas condensadas em cada espécie, $n_{01}=N_{01} / N$ e $n_{02}=N_{02} / N$ e os estados coletivos do sistema. Para uma temperatura, $T$, diferente de zero, iniciamos a rotina assumindo como dados de entrada os autovalores e autovetores da equação de HartreeFock-Bogoliubov-Popov calculados na temperatura anterior. Com esses dados calculamos as depleçöes, $d_{\alpha \beta}$, e, com elas, determinamos os novos parâmetros de controle da equação de Gross-Pitaeviskii e, ao resolvê-la, encontramos novas frações de partículas condensadas em cada espécie e a fração do total de partículas condensadas no sistema, $n_{0}=n_{01}+$ $n_{02}$. Usamos a fração total para comparar com a calculada na iteração anterior e caso o erro relativo seja menor que $0.1 \%$, assumimos os resultados do passo anterior como solução do sistema de equações, caso contrário construímos os elementos de matriz de $\eta \mathcal{H}$, diagonalizamos essa matriz e com os autovalores e autovetores iniciamos novamente o ciclo. Continuamos esse procedimento tantas vezes quanto forem necessárias para obter um erro relativo na fração total de partículas no condensado inferior ao estabelecido.

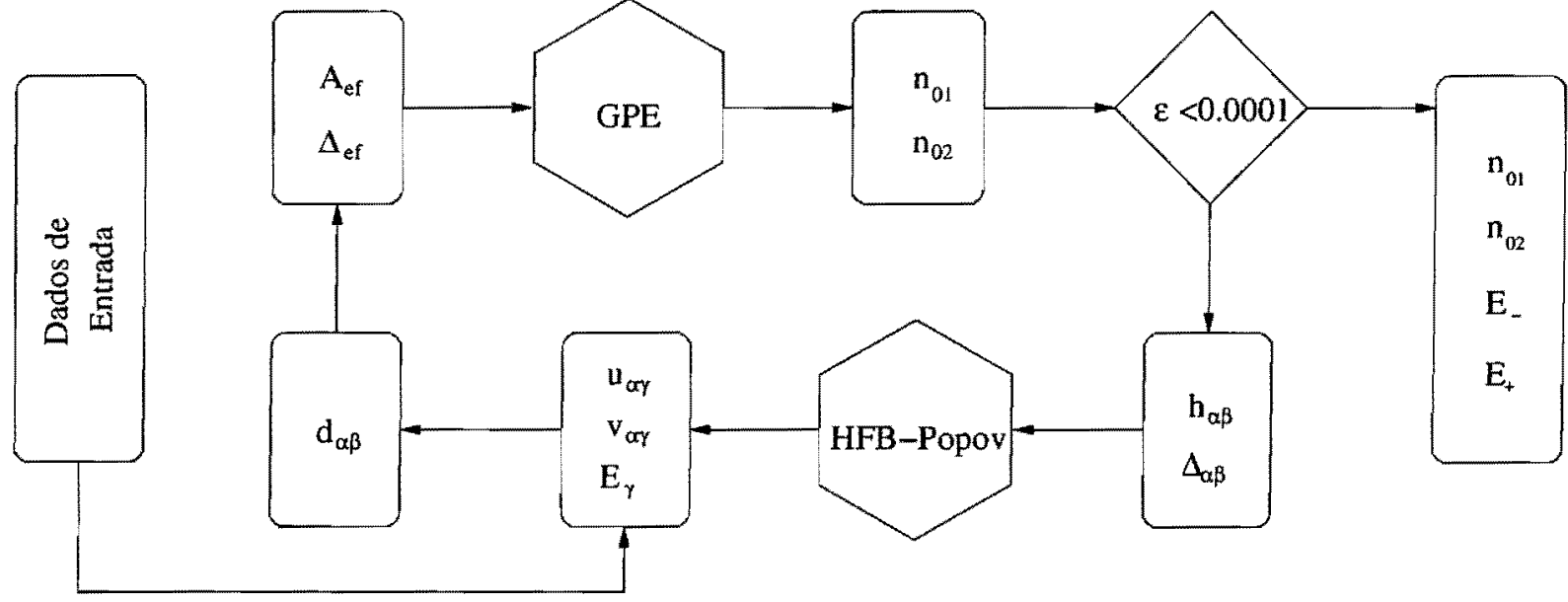

Figura 3.1: O fluxograma da rotina.

Na temperatura nula, os dados de entrada são os autovalores e autovetores calculados de acordo com a teoria de Bogoliubov [70]. Com esses dados de entrada, calculamos as depleções e procedemos da maneira descrita para temperatura diferente de zero.

No nosso procedimento, partindo da temperatura nula, resolvemos a equação de Gross- 
Pitaevskii e as equações de Hartree-Fock-Bogoliubov-Popov em função da temperatura até atingirmos a temperatura de transição, ou seja, a temperatura em que, a partir dela, a densidade de partículas no condensado é nula. Como resultado do cálculo, temos dois espectros de excitação coletiva sendo um com uma lacuna para momento nulo e outro sem essa lacuna, seus respectivos modos e as frações de partículas condensadas em cada nível hiperfino como função da temperatura.

Consideramos o caso na qual a fase relativa entre os condensados seja $\pi$, pois os mínimos locais de energia sempre ocorrem para essa diferença de fase [70]. Dependendo do valor dos parâmetros de controle, a equação de Gross-Pitaevskii tem uma solução estável ou três soluções, duas estáveis e uma instável. Na figura 3.2, destacamos as duas regiões, a região branca tendo uma solução, a cinza três e a curva que delimita essas regiões é dada por

$$
\Delta_{e f}= \pm\left(A_{e f}^{2 / 3}-1\right)^{3 / 2}
$$

onde a solução positiva bifurca na curva com sinal positivo e a negativa na curva com sinal negativo.

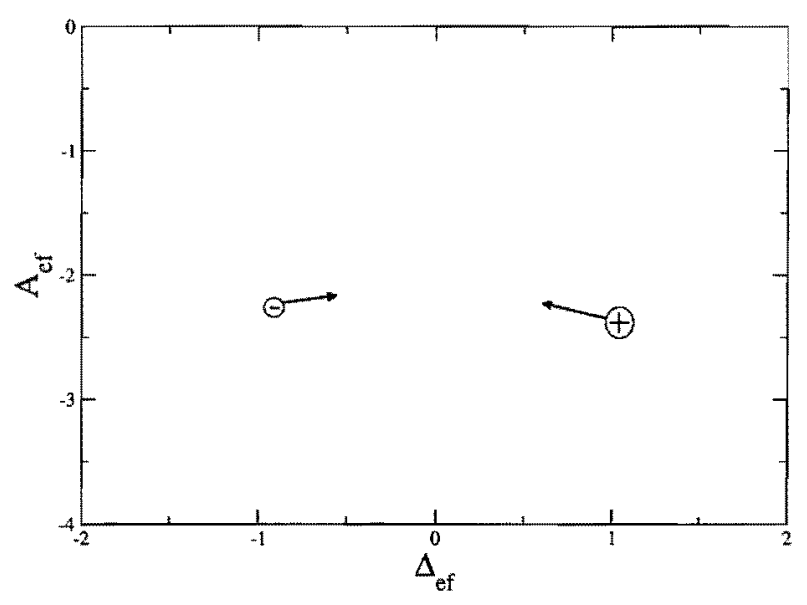

Figura 3.2: Gráfico do espaço de parâmetros de controle da equação de Gross-Pitaevskii. $\mathrm{Na}$ região cinza existe três soluções da equação de Gross-Pitaevskii, duas estáveis e uma instável e na branca uma única soluçāo. Na curva $(+)$ ocorre a bifurcação da solução positiva e na curva (-) a da solução negativa (ver equação (3.1)).

Ao resolver a equação de Gross-Pitaevskii (2.79), teremos uma única solução, $\bar{f}$, caso o par ordenado composto pela dessintonia efetiva e pela interação mútua efetiva, $\left(\Delta_{e f}, A_{e f}\right)$, 
esteja na região branca do gráfico. Essa solução será positiva se a dessintonia efetiva for negativa, e negativa se for positiva. Caso o par ordenado esteja na região cinza, a equação de Gross-Pitaevskii terá duas soluções estáveis, uma positiva e outra negativa.

No nosso caso, os parâmetros de controle dependem da temperatura. Desse modo, quando variamos a temperatura a partir da temperatura nula, os parâmetros de controle da equação de Gross-Pitaevskii descrevem uma curva no plano $\Delta_{e f} \times A_{e f}$. Com isso, podemos investigar a dependência da bi-estabilidade com a temperatura a partir da análise da curva que o sistema descreve nesse plano.

Aplicamos o método da solução da equação de Gross-Pitaevskii e das equações de Hartree-Fock-Bogoliubov-Popov para uma mistura de átomos de ${ }^{87} R b$ em dois estados hiperfinos, um estado correspondendo a $F=2$ e $m_{F}=1,|1\rangle=|2,1\rangle$, e outro correspondendo a $F=1$ e $m_{F}=-1,|2\rangle=|1,-1\rangle$. A dessintonia do campo de radiação é igual a $\delta=\hbar\left(\omega_{21}-\omega_{1-1}\right)-\hbar \omega[30]$, onde $\hbar \omega_{F m_{F}}$ são as energias de um átomo no estado hiperfino $\left|F, m_{F}\right\rangle$ e $\omega$ é a soma das freqüências dos dois fótons envolvidos no processo. Nessa tese consideramos a dessintonia como sendo nula.

Por conveniência, medimos a energia e o comprimento em unidades de $\hbar^{2} / 2 m a^{2}$ e $a$ respectivamente, com $a=100 a_{12}$, com $a_{12}$ sendo o comprimento de espalhamento de onda $s$ entre átomos em estados hiperfinos diferentes. Assim, dado das referências $[30,31]$ a massa do ${ }^{87} R b, m=1.4432 \times 10^{-25} \mathrm{~kg}$, e o comprimento de espalhamento, $a_{12}=5.5(3) \times 10^{-9} \mathrm{~m}$, vemos que a unidade de energia nessa escala é $\frac{\hbar^{2}}{2 m a^{2}}=h(192 \mathrm{~Hz})$.

Três casos foram considerados, o primeiro correspondendo a uma escolha onde os parâmetros do sistema são próximos dos valores experimentais e outros dois escolhidos de modo a propiciar a investigação da dependência da bi-estabilidade com a temperatura.

Em cada um dos casos teremos como objetivos investigar a dependência com a temperatura da população dos condensados, da lacuna e da velocidade do som do espectro de excitações, determinar a temperatura de transição onde a população dos condensados se anula e, para os dois últimos casos, examinar como a bi-estabilidade depende da temperatura. 


\subsection{Um caso "realista"}

Para simularmos algo mais próximo da experiência, fixamos os comprimentos de espalhamento de onda $s$ de acordo com os valores experimentais [30,31]. Dessas referências, sabemos que o comprimento de espalhamento entre átomos de ${ }^{87} R b$ no estado hiperfino $|1\rangle=\left|F=2, m_{F}=1\right\rangle$ e $|2\rangle=|1,-1\rangle$ é da ordem de nanometros, $a_{12}=5.5(3) \times 10^{-9} \mathrm{~m}$ e as partículas no mesmo estado hiperfino têm uma interação cujo comprimento de espalhamento é de $a_{11}=0.97 a_{12}$ para o primeiro estado hiperfino e de $a_{22}=1.03 a_{12}$ para o segundo. Para fixarmos o hamiltoniano de muitos corpos (2.1), consideramos $J / h=192 \mathrm{~Hz}$ e $\delta=0$.

No intervalo de densidades que esses condensados ocorrem $10^{11} \mathrm{~cm}^{-3}<\rho<10^{15} \mathrm{~cm}^{-3}$, escolhemos um regime suficientemente diluído, $\rho \approx 10^{13} \mathrm{~cm}^{-3}$, para que o parâmetro do gás seja $\rho a_{12}^{3}=10^{-6}$.

Acidentalmente, esses valores de comprimentos de espalhamento fazem com que a interação mútua efetiva, $A_{e f}$, seja nula. Entretanto, a dessintonia efetiva, para uma temperatura nula, é negativa, $\Delta_{e f}=-0.0038$, e decresce com o aumento da temperatura até alcançar o valor de $\Delta_{e f}=-0.0075$, na temperatura de transição. No espaço de parâmetros de controle, vemos que a curva que descreve a sua variação com a temperatura corresponde a um segmento de reta na região onde existe apenas um estado de equilíbrio, figura 3.3.

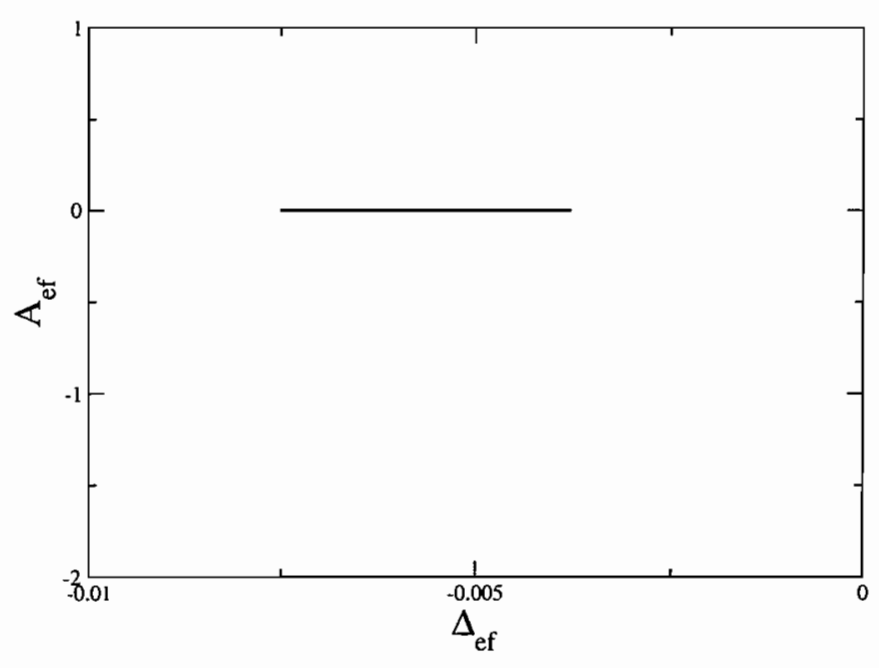

Figura 3.3: Gráfico da curva descrita pelos parâmetros de controle quando variamos a temperatura desde zero, $T=0$, até a temperatura de transição, $T=T_{t}$. 
$\mathrm{Na}$ região onde a curva se situa, podemos resolver a equação de Gross-Pitaevskii exatamente e a solução é dada por

$$
\bar{f}=-\frac{\Delta_{e f}}{\sqrt{1+\Delta_{e f}^{2}}} .
$$

Como o módulo da dessintonia efetiva é muito menor que a unidade $\left|\Delta_{e f}\right| \ll 1$, o valor da solução da equação de Gross-Pitaevskii é, também, pequeno, $|\bar{f}| \ll 1$, isso provoca uma diferença diminuta entre as populações de partículas condensadas em cada estado hiperfino. Na figura 3.4 vemos que, independente da temperatura, a fração de partículas condensadas no primeiro estado hiperfino quase não se distingüe da fração de partículas condensadas no segundo estado.

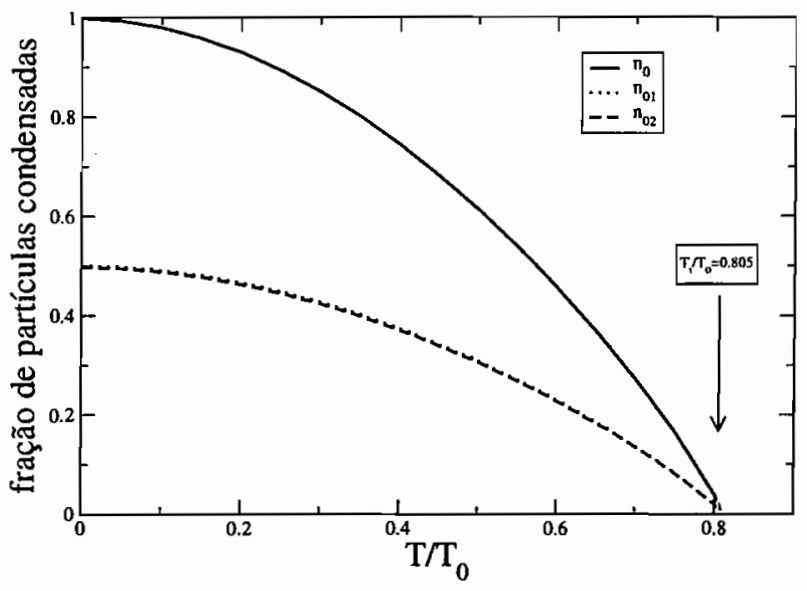

Figura 3.4: Gráfico da fração total e de cada espécie de partículas condensadas como função da temperatura. A seta indica o valor da temperatura de transição, $T_{t}$, calculado analiticamente.

Nessa mesma figura vemos que, ao aumentarmos a temperatura do sistema, a fração do total de partículas condensadas e as frações de cada espécie decrescem e tendem a zero, $n_{0}, n_{01}$ e $n_{02} \rightarrow 0$, para uma determinada temperatura, $T_{t}$. Notamos que, devido à característica coerente do acoplamento Josephson entre os estados hiperfinos, as populações dos condensados se anulam simultaneamente e a temperatura de transição é menor que a temperatura de transição para o gás livre com densidade igual a da mistura, $T_{0}$, dado 


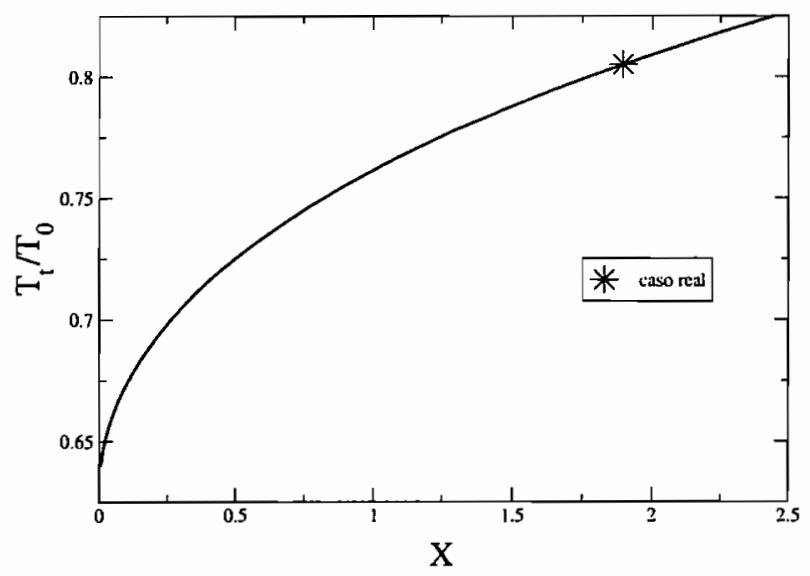

Figura 3.5: Gráfico da temperatura de transição como função de $X$, equação (B.24), onde $X=\frac{2 J_{e f}}{k_{B} T_{0} \sqrt{1-\bar{f}^{2}}}$. A estrela no gráfico denota o resultado da solução numérica das equações de Gross-Pitaevskii e Hartree-Fock-Bogoliubov-Popov.

em (B.10). Para o caso "realista" a temperatura transição do sistema corresponde a $T_{t} / T_{0}=0.8$

$\mathrm{Na}$ temperatura de transição, não há um número macroscópico de átomos no estado de momento nulo. Com isso, conhecendo o Josephson efetivo e a solução da equação de Gross-Pitaevskii, podemos calcular exatamente a temperatura de transição pela equação transcendental (B.19) que, naturalmente, concorda com a calculada através da solução numérica das equação de Gross-Pitaevskii e Hartree-Fock-Bogoliubov-Popov, ver figura 3.5 .

Também calculamos as energias dos modos de excitação coletiva do sistema e vemos que, independente da temperatura, sempre temos dois ramos de excitação, um com uma lacuna no limite em que o momento seja nulo, e outro sem a lacuna. Na figura 3.6 mostramos um caso no qual $T / T_{0}=0.3$.

Esses dois ramos têm aspectos semelhantes aos existentes nos modos de excitação coletiva de um cristal diatômico unidimensional. No espectro de excitação coletiva desse sólido, identificamos um ramo com lacuna, conhecido como ramo óptico, e outro sem, ramo acústico [75]. No ramo acústico temos um comportamento linear na região de momento pequeno e sua inclinação se relaciona com a velocidade do som do sólido. No ramo óptico temos uma lacuna correspondendo a energia necessária para que os átomos de espécies diferentes oscilem com fase relativa igual a $\pi$. A analogia que podemos fazer 


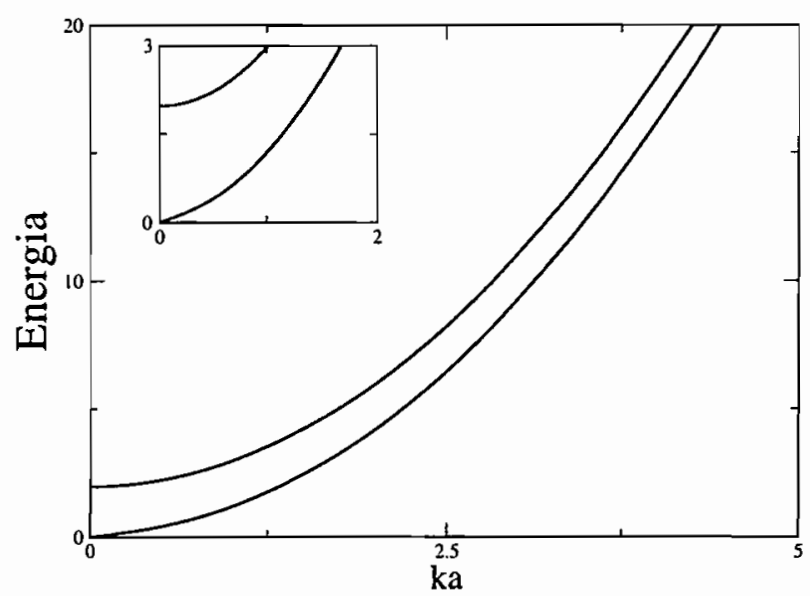

Figura 3.6: Os dois ramos de excitação coletiva numa temperatura de $T / T_{0}=0.3 \mathrm{em}$ unidades de $\hbar^{2} / 2 m a^{2}$. E uma ampliação do gráfico para eventual observação do ramo acústico.

com os espectros, por nós determinado, limita-se a associar os nomes de ramo óptico e acústico aos ramos do espectro de excitação e identificar, nos respectivos ramos, a lacuna e a velocidade do som.

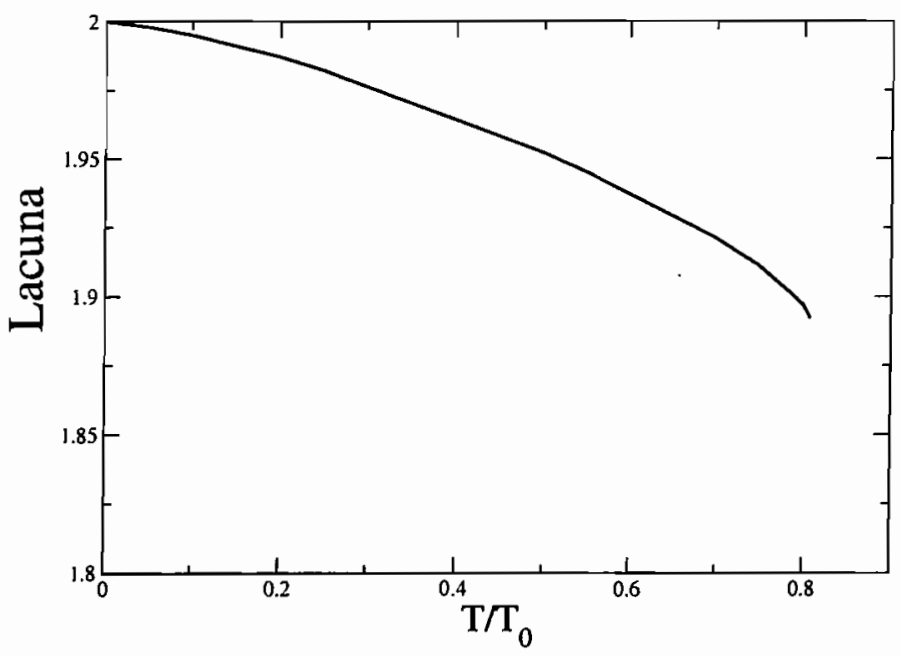

Figura 3.7: Gráfico da lacuna do ramo óptico em unidades de $\hbar^{2} / 2 m a^{2}$ como função da temperatura.

Caracterizamos o ramo óptico por sua lacuna no limite de momento nulo e vemos, no 
gráfico 3.7, o comportamento dessa em função da temperatura. Notamos que a lacuna decresce monotonicamente quando aumentamos a temperatura, sendo finita na temperatura de transição. Comparamos os valores da lacuna obtido pelo cálculo numérico, com os valores obtidos da expressão analítica (2.103) e verificamos que eles coincidem.

Extraímos a velocidade do som a partir da inclinação do espectro do ramo acústico no limite de momento nulo. Esses valores foram determinados através do limite de momento nulo da derivada da energia do ramo acústico em relação ao momento e verificados pela expressão analítica (2.112). No gráfico 3.8 observamos um acentuado decréscimo da velocidade do som quando a temperatura aproxima-se da temperatura de transição. Pelo cálculo analítico da velocidade do som, expressão (2.112), vemos que ela se anula na temperatura de transição por ser proporcional a densidade de partículas no condensado.

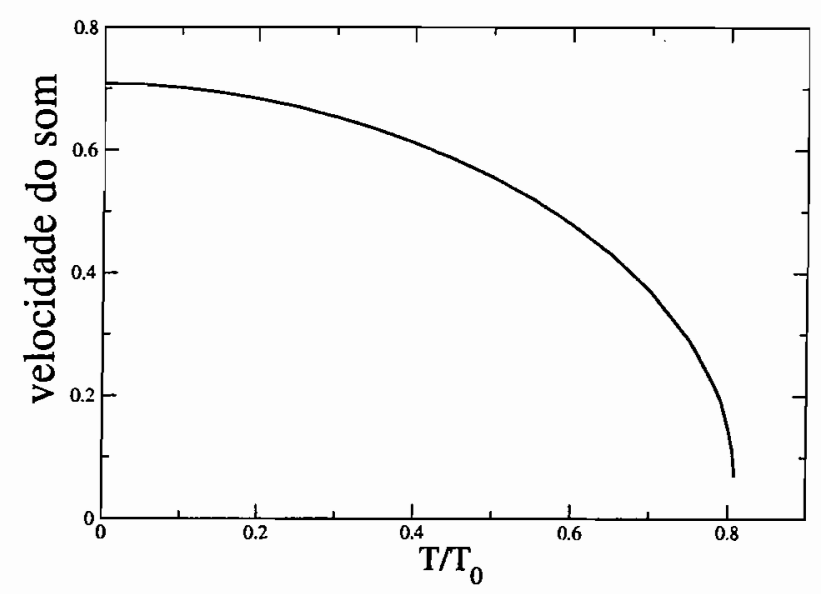

Figura 3.8: Gráfico da velocidade do som extraída do ramo acústico em unidades de $\hbar / 2 m a$ como função da temperatura. 


\subsection{Um caso na região de bi-estabilidade com uma dessintonia efetiva diferente de zero}

Procuramos estudar um sistema cujos parâmetros de controle, para temperatura nula e depleção desprezível, estejam na região de bi-estabilidade. A priori, devido a interação mútua efetiva ser proporcional a densidade de partículas condensadas, vemos que, com a temperatura aproximando-se da temperatura de transição, a interação mútua efetiva, $A_{e f}$, anula-se. Desse modo, a curva que descreve a evolução dos parâmetros de controle com a temperatura necessariamente sai da região de duas soluções estáveis de Gross-Pitaevskii e entra na de uma única solução. Quando isso ocorre, pode ser que uma das soluções estáveis colapsa e, portanto, desaparece a bi-estabilidade.

Com o objetivo de localizar o sistema na região de bi-estabilidade, alteramos os parâmetros do caso "realista" para que os novos parâmetros de controle da Gross-Pitaevskii estejam, em temperatura nula, na região de duas soluções estáveis. Para isso, escolhemos um parâmetro do gás uma ordem de grandeza maior do que no caso "realista", $\rho a_{12}^{3}=$ $10^{-5}$, mudamos as proporções dos comprimentos de espalhamento para $a_{11}=0.375 a_{12}$ e $a_{22}=0.125 a_{12}$, permanecemos com a dessintonia nula e diminuímos a freqüência de Rabi que acopla os dois estados hiperfinos para dezenas de Hertz, $J / h \approx 60 \mathrm{~Hz}$. Dessa forma, temos um sistema cuja interação mútua e a dessintonia efetiva, num regime de temperatura e depleção nula, são iguais a $A_{e f}=-3$ e $\Delta_{e f}=0.5$ respectivamente; o que localiza o sistema na região de bi-estabilidade, $A_{e f}<-1$ e $-\left|\Delta_{c r}\right|<\Delta_{e f}<\left|\Delta_{c r}\right|$, com $\Delta_{c r}\left(A_{e f}=-3\right)=1.12$. Assim a equação de Gross-Pitaevskii tem duas soluções estáveis, uma positiva, $\bar{f}_{+}=0.91$, e outra negativa, $\bar{f}_{-}=-0.96$.

Resolvendo auto-consistentemente as equações de Gross-Pitaevskii e de Hartree-FockBogoliubov-Popov na região de bi-estabilidade, achamos duas soluções estáveis: uma onde a fração, $\bar{f}$, é positiva e outra que é negativa. Como os parâmetros de controle dependem dos estados temos, como função da temperatura, curvas distintas para cada uma dessas duas soluções. Quando a temperatura cresce, a partir da temperatura nula, vemos que a solução positiva aproxima-se da curva de bifurcação das soluções positivas, curva $(+)$, e desaparece como mostrado na figura 3.9.

No nosso cálculo, tivemos problemas numéricos para atingir a temperatura de colapso pois na região próxima dessa temperatura, $T / T_{0}=0.29$, uma pequena variação da mesma 
faz com que a solução positiva desapareça. Na figura 3.12, temos uma evidência da dificuldade numérica mencionada pois a lacuna do ramo óptico decresce rapidamente quando nos aproximamos da curva de bifurcação, porém não atingimos a temperatura de colapso pois, como mostrado em [70], a expressão analítica da lacuna (2.103) é proporcional a segunda derivada da energia e se anula na curva de bifurcação. A nossa conclusão, discutida adiante, afirma que a solução positiva deixa de existir pelo fato do sistema ficar instável antes do colapso, como sugerido na referência [70] em um outro contexto.

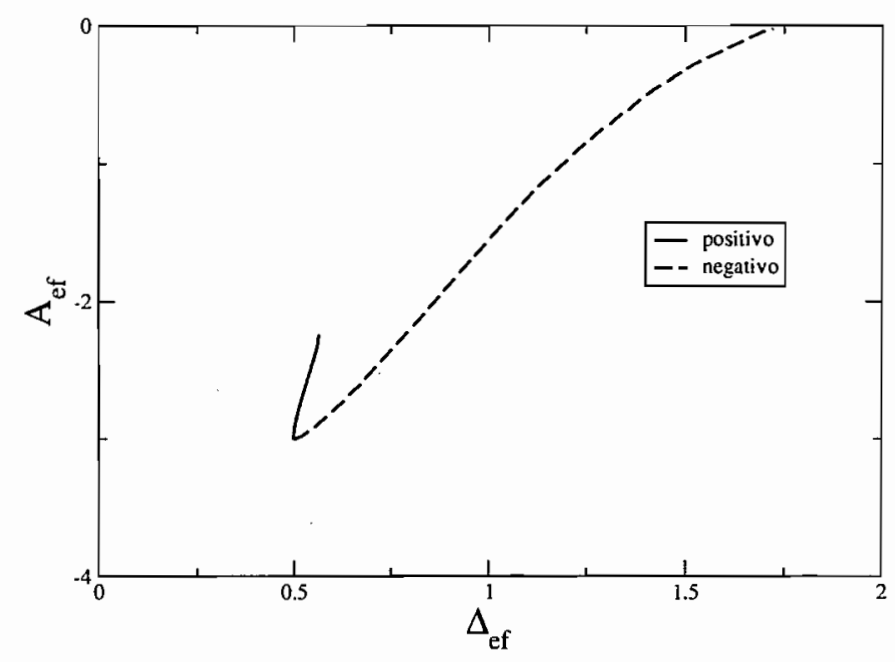

Figura 3.9: Gráfico da interação mútua efetiva como função da dessintonia efetiva.

Para a solução negativa, vemos que a curva de evolução dos parâmetros de controle com a temperatura afasta-se da curva de bifurcação (-), cruza a curva (+) na temperatura de $T / T_{0}=0.29$ e é a única solução para temperaturas maiores que essa temperatura. Quando o sistema aproxima-se da temperatura de transição, a interação mútua efetiva tende a zero e a dessintonia efetiva nessa temperatura é maior do que para temperatura nula, $\Delta_{e f}=1.75$.

Ao observar o gráfico da fração de partículas em função da temperatura, figura 3.10, para a solução positiva, vemos que a fração de partículas no segundo estado hiperfino cresce quando aumentamos a temperatura, enquanto a fração total de partículas condensadas e de partículas no primeiro estado hiperfino decresce. Para temperaturas maiores que a temperatura de colapso, só existe um estado de equilíbrio que corresponde à solução 
negativa. Essa solução apresenta uma temperatura de transição de $T_{t} / T_{0}=0.69$ e tem um comportamento muito semelhante ao caso "realista", ver figura 3.11.

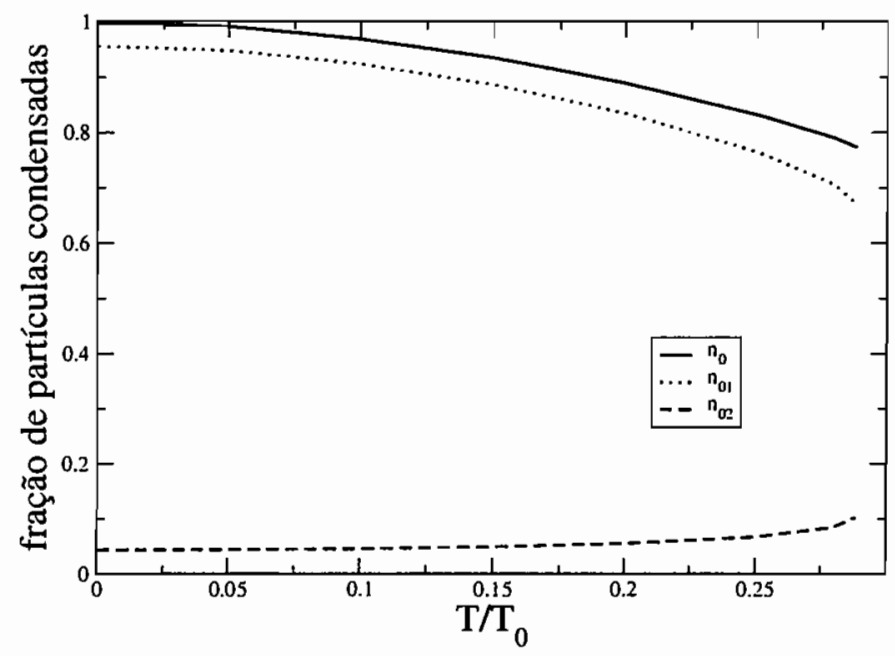

Figura 3.10: Gráfico da fração total e de cada espécie de partículas condensadas como função da temperatura para a solução positiva.

Na figura 3.12, onde apresentamos o gráfico da lacuna como função da temperatura tanto para a solução positiva quanto para a negativa, vemos que a lacuna da solução positiva decresce a medida que nos aproximamos da temperatura de colapso. Esse é o comportamento esperado pois, quando a solução positiva aproxima-se da curva de bifurcação $(+)$, a lacuna, que é proporcional a derivada segunda da energia interna [70], tende a zero. Para a lacuna da solução negativa temos um comportamento parecido com a lacuna da sessão anterior. Ambas curvas coincidem com a expressão analítica (2.110).

Na figura 3.13, também, apresentamos os gráficos da velocidade do som como função da temperatura tanto para a solução positiva quanto para a negativa. No caso da solução positiva vemos que a velocidade do som decresce rapidamente quando a temperatura aproxima-se do valor de colapso. Por problemas numéricos, não conseguimos estabelecer se a velocidade do som tende a zero antes da curva que corresponde a solução positiva alcance a curva de bifurcação, indicando que o sistema fica instável antes de ocorrer o cruzamento.

Entretanto, uma análise qualitativa baseada na expressão analítica para a velocidade 

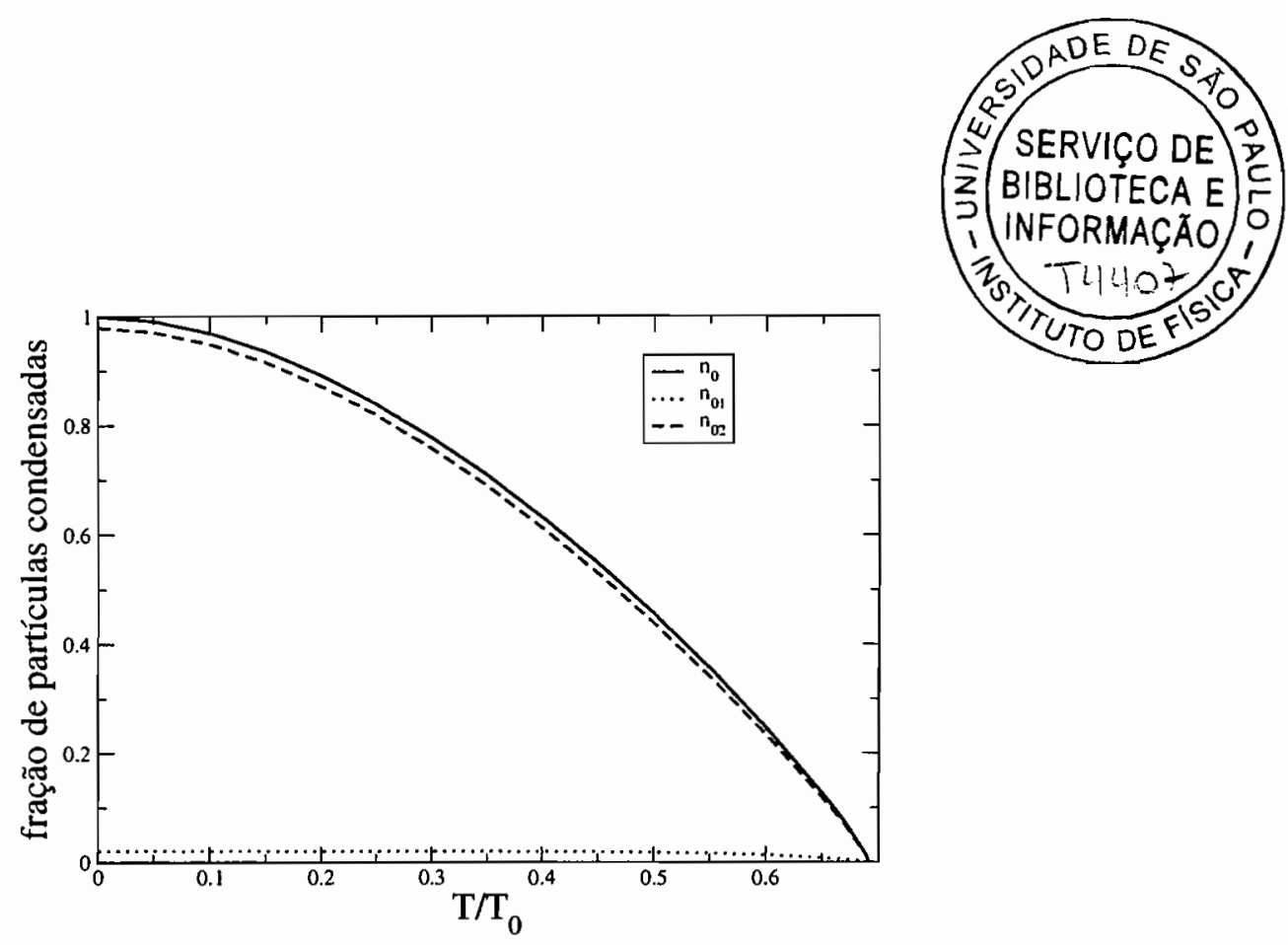

Figura 3.11: Gráfico da fração total e de cada espécie de partículas condensadas como função da temperatura para a solução negativa.

do som, (2.112), sugere que o sistema fica instável antes de atingir a temperatura de colapso, em concordância com a referência [70]. Com efeito, como a lacuna tende a zero quando nos aproximamos da curva de bifurcação, vemos que, pela expressão para $v_{s}^{2}$ (2.111), o quadrado da velocidade do som diverge com o sinal da divergência dado por $c_{2}$. Como o cálculo numérico mostra que $v_{s}^{2}$ diminui, concluímos que $v_{s}^{2} \rightarrow-\infty$ na temperatura de colapso da solução positiva e, por conseguinte, se anula e assume valores negativos antes de ocorrer o cruzamento. Sendo assim, a solução positiva fica instável antes de atingir a curva de bifurcação. Porém, observamos que isso ocorre para temperaturas muito próximas a temperatura de colapso.

Para a solução negativa a velocidade do som decresce com a temperatura tendendo a zero na temperatura de transição como esperado. O cálculo usando a expressão analítica para a velocidade do som, (2.112), ajusta-se com os dados das curvas na figura 3.13.

Um exame das equações de Gross-Pitaevskii e de Hartree-Fock-Bogoliubov-Popov mostra-nos que, dada uma solução com a interação mútua efetiva negativa, $A_{e f}<0$, e a dessintonia efetiva positiva, $\Delta_{e f}>0$, existe uma outra solução onde as densidades, as depleções e os parâmetros da interação são trocados, $\rho_{01} \rightleftharpoons \rho_{02}, d_{11} \rightleftharpoons d_{22}$ e $\lambda_{11} \rightleftharpoons \lambda_{22}$. Nesse caso a interação mútua efetiva permanece invariante, $A_{e f} \rightarrow A_{e f}$, a dessintonia 


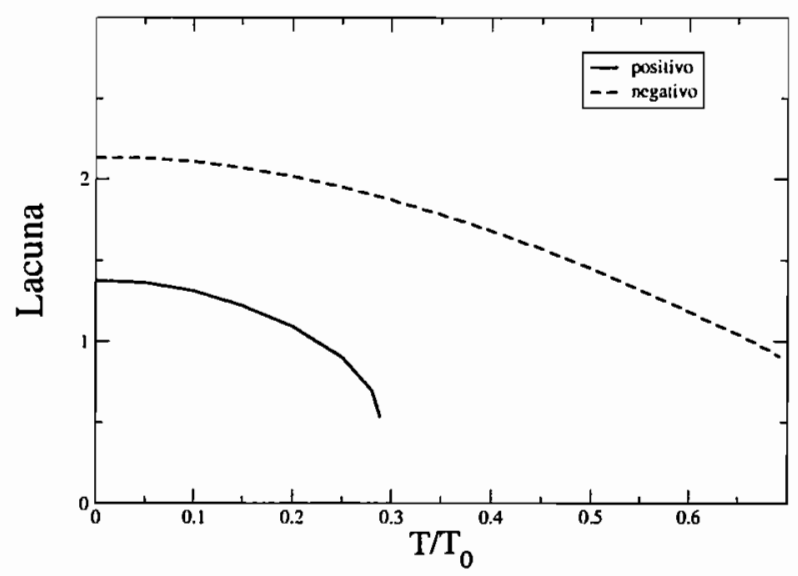

Figura 3.12: Gráfico da lacuna em unidades de $\hbar^{2} / 2 m a^{2}$ como funcão da temperatura.

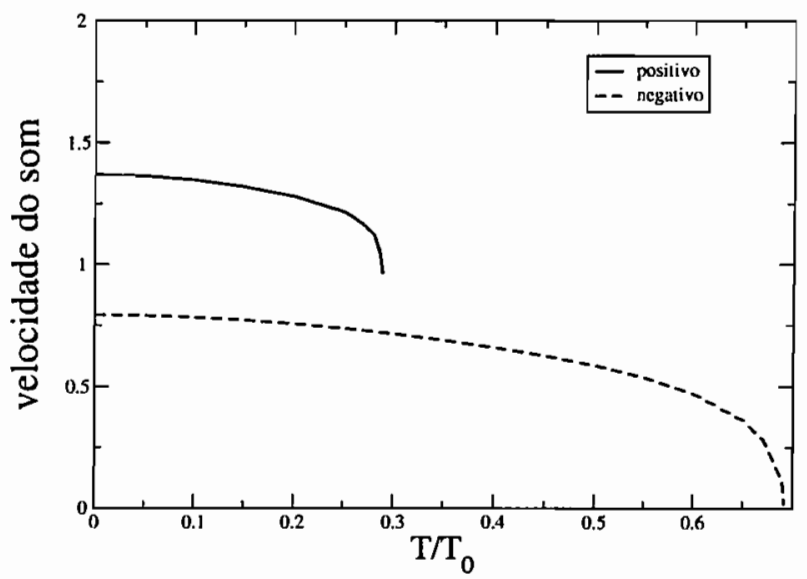

Figura 3.13: Gráfico da velocidade do som em unidades de $\hbar / 2 m a$ como função da temperatura.

efetiva troca de sinal, $\Delta_{e f} \rightarrow-\Delta_{e f}$, e, por conseguinte, temos a troca de sinal da solução da equação de Gross-Pitaevskii, $\bar{f} \rightarrow-\bar{f}$. Desse modo, a curva no espaço dos parâmetros de controle, para esse novo conjunto de parâmetros do sistema, é a reflexão em torno do eixo da interação mútua efetiva, $A_{e f}$, da curva da figura 3.9 , isto é, $\left(A_{e f}, \Delta_{e f}, \bar{f}\right)$ e $\left(A_{e f},-\Delta_{e f},-\bar{f}\right)$ são pares de soluções.

Quanto a equações de Hartree-Fock-Bogolibov-Popov, vemos que o espectro das excitações coletivas é invariante pela troca dos parâmetros dos estados hiperfinos. Desse modo, o comportamento, como função da temperatura, das grandezas físicas são iguais às do caso anterior, lembrando que troca-se o sinal da solução. Exemplificando, para 
esse caso, o estado de equilíbrio que desaparece é o correspondente à solução negativa, a solução positiva permanecendo estável até atingir a temperatura de transição.

Resumindo, devido à bi-estabilidade, o que acontece com o sistema quando aumentamos a temperatura, a partir de zero, depende de sua história. Ao seguir a solução positiva, existe uma temperatura de colapso para a qual essa solução desaparece e o estado de equilíbrio muda repentinamente para a solução negativa que é o único estado de equilíbrio para temperaturas maiores que a temperatura de colapso. Entretanto, ao esfriarmos o sistema, ele sempre percorre a curva da solução negativa mesmo quando a temperatura fica abaixo da temperatura de colapso. Esse fenômeno de histerese do sistema é uma conseqüência da sua bi-estabilidade. 


\subsection{Um caso na região de bi-estabilidade com uma dessintonia nula}

Mudando somente as razões entre os comprimentos de espalhamento, $a_{11}=0.25 a_{12} \mathrm{e}$ $a_{22}=0.25 a_{12}$, e mantendo os demais parâmetros, temos, na temperatura nula e sem considerar as depleções, um sistema com interação mútua efetiva igual ao da secção anterior porém com a dessintonia efetiva igual a zero.

Diferentemente do caso anterior, nenhuma das duas soluções da equação de GrossPitaeviskii desaparece apesar de estarmos na região de bi-estabilidade, ver figura 3.14. De fato, vemos que, nesse caso limite, as curvas que descrevem a evolução dos parâmetros da Gross-Pitaevskii estão em regiões onde o sinal da dessintonia efetiva é oposto ao das suas respectivas soluções. Desse modo, não existe o desaparecimento das soluções pois as curvas ao deixar a região de bi-estabilidade não cruzam as respectivas curvas de bifurcação.

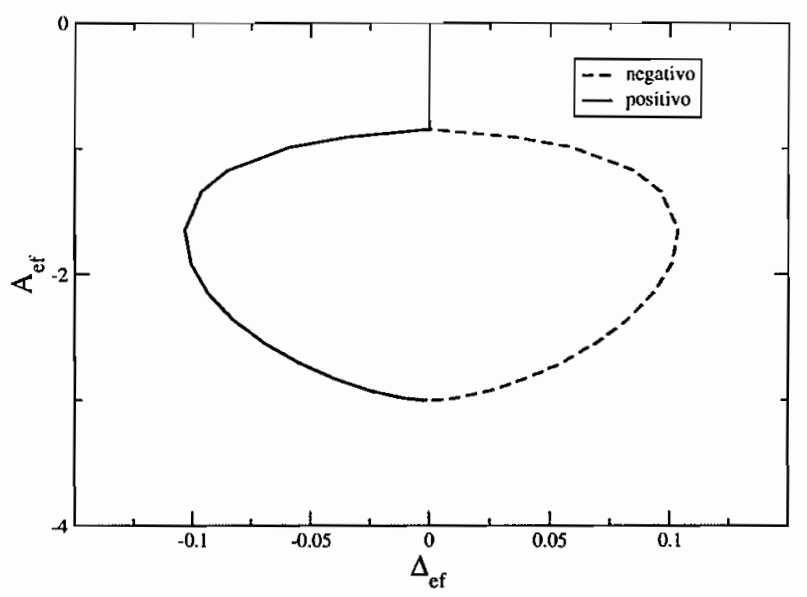

Figura 3.14: Gráfico da interação mútua efetiva como função da dessintonia efetiva. As curvas estão relacionadas por uma reflexão em torno do eixo $O_{A_{e f}}$.

As duas soluções da equação de Gross-Pitaevskii, para uma determinada temperatura, têm valores iguais em módulos diferindo somente pelo sinal de cada solução. Isso se deve ao fato das curvas no espaço dos parâmetros de controle de cada solução estarem relacionadas por uma reflexão em torno do eixo $O_{A_{e f}}$, isto é, $\left(A_{e f}, \Delta_{e f}, \bar{f}\right)$ e $\left(A_{e f},-\Delta_{e f},-\bar{f}\right)$ são pares de soluções. Se continuarmos a aumentar a temperatura, as duas soluções degeneram-se 
indo ambas a zero para uma temperatura em torno de $T / T_{0}=0.57$. Nessa temperatura a interação mútua efetiva é maior que -1 e a dessintonia efetiva se anula. Na figura 3.15 vemos as soluções negativa e positiva da equação de Gross-Pitaevskii indo à zero drasticamente nessa temperatura, havendo um ponto onde as duas soluções estáveis coincidem. Para temperaturas maiores que essa temperatura de degenerescência das soluções, temos apenas uma única solução da equação de Gross-Pitaevskii, $\bar{f}=0$.

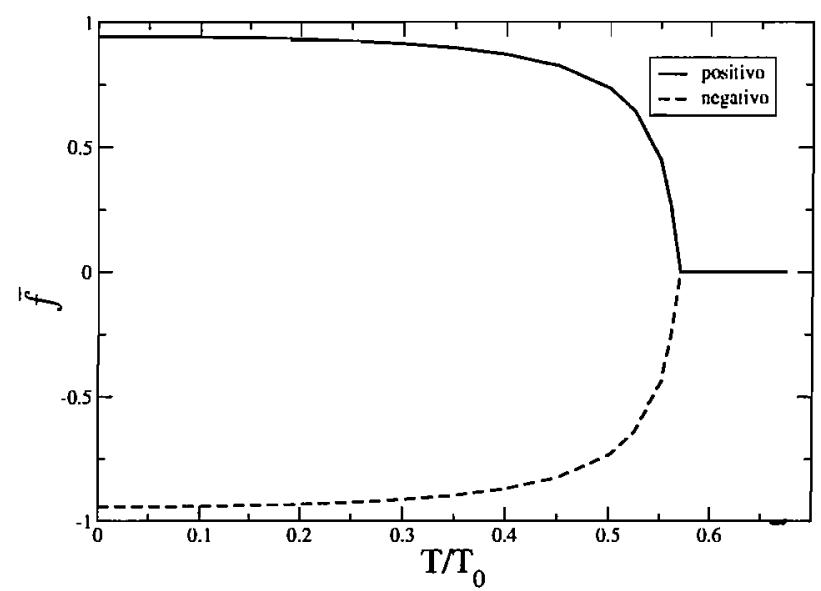

Figura 3.15: Gráfico das frações relativas de átomos nos condensados como função da temperatura.

Com as soluções da equação de Gross-Pitaevskii se anulando nessa temperatura, vemos que as populações de ambas espécies assumem os mesmo valores, ver figura 3.16. Note que, na temperatura da degenerescência, a derivada das frações relativas é descontínua. Nesse mesmo gráfico podemos ver que a temperatura de transição é de $T_{t} / T_{0}=0.67$.

No caso das energias de excitação, vemos que elas coincidem para as duas soluções. A razão dessa igualdade está na propriedade de, quando $\lambda_{11}=\lambda_{22}$ e para uma determinada temperatura, as equações que determinam os espectros de excitação dependerem do quadrado da fração relativa, ver equação (2.109). Assim, vemos que os espectros de excitação da solução positiva da equação de Gross-Pitaevskii é idêntico ao da solução negativa e, portanto, tanto a lacuna quanto a velocidade do som são as mesmas independente do sinal da solução da equação de Gross-Pitaevskii.

Nas figuras 3.17 e 3.18 vemos o comportamento da lacuna do ramo óptico e da veloci- 

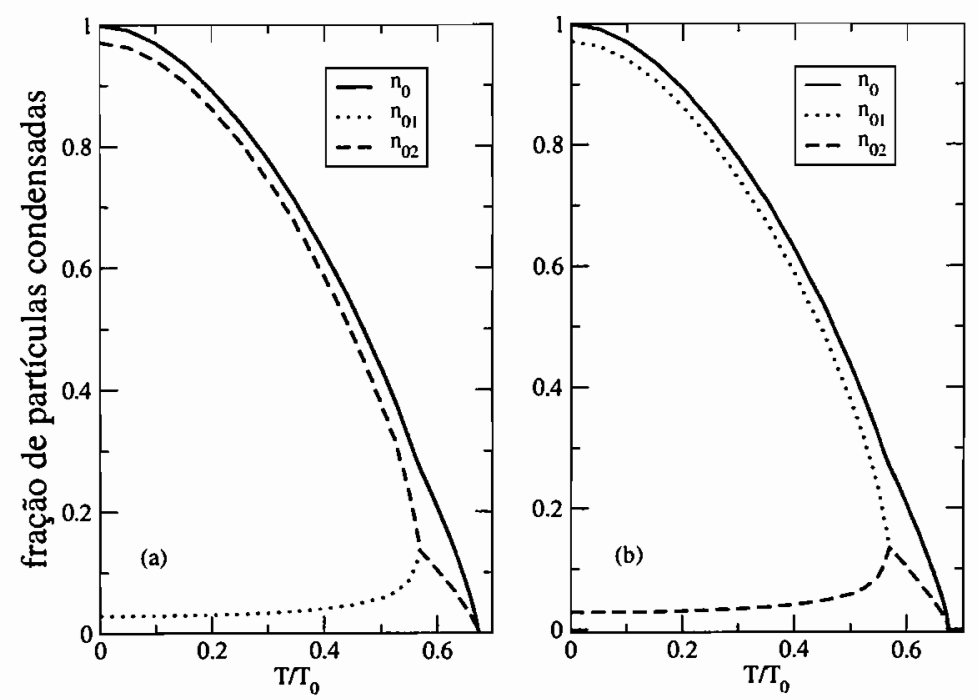

Figura 3.16: Gráfico da fração total e de cada espécie de partículas condensadas como função da temperatura escolhendo a solução negativa (a) ou a positiva (b).

dade do som extraída do ramo acústico como função da temperatura. Observamos uma descontinuidade na derivada em relação à temperatura dessas duas grandezas na temperatura de degenerescência, correspondendo a descontinuidade na derivada das frações relativas. Como nos casos anteriores, na temperatura de transição a lacuna é finita e a velocidade do som é nula. Verificamos que ambas curvas coincidem com as soluções analíticas (2.103) e (2.112) respectivamente. 


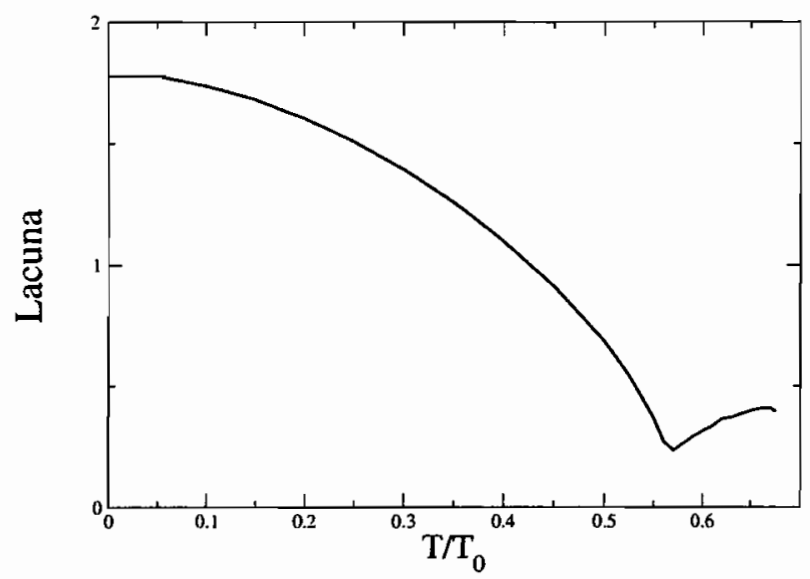

Figura 3.17: Gráfico da lacuna do ramo óptico em unidades de $\hbar^{2} / 2 m a^{2}$ como função da temperatura.

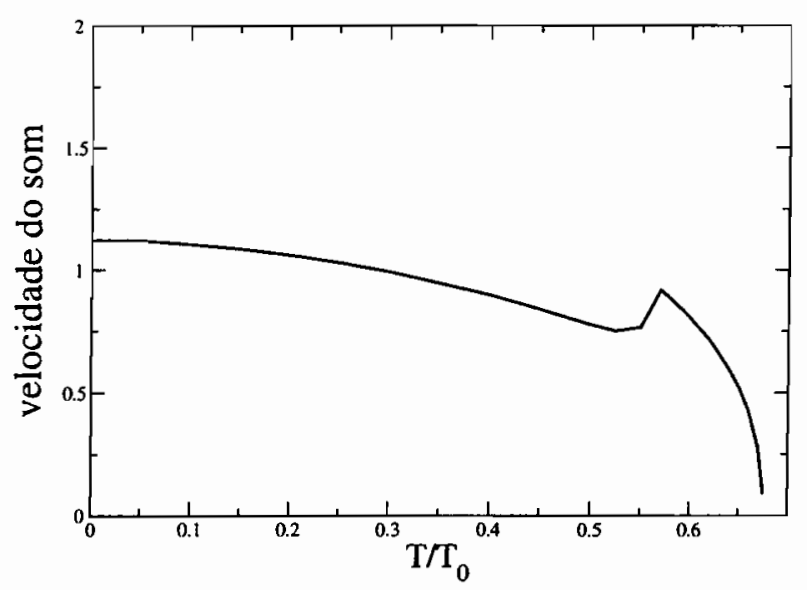

Figura 3.18: Gráfico da velocidade do som em unidades de $\hbar / 2 m a$ como função da temperatura. 


\section{Capítulo 4}

\section{Conclusão}

Usando o princípio de máxima entropia, deduzimos as equações de Gross-Pittaevskii, para os estados de equilíbrio, e as equações de Hartree-Fock-Bogoliubov, para as excitações coletivas, de uma mistura homogênea de átomos bosônicos em dois estados hiperfinos diferentes acoplados por uma interação de Josephson interno e a uma temperatura finita.

Do mesmo modo que em condensados com apenas uma componente, os dois ramos do espectro de energia na teoria de Hartree-Fock-Bogoliubov têm uma lacuna, o que viola o teorema de Hugenholtz-Pines. Mostramos como contornar esse problema fazendo uma aproximação conhecida na literatura como aproximação de Popov que despreza a contribuição das densidades de pares (densidades anômalas) nas equações de Gross-Pitaevskii e nas de Hartree-Fock-Bogoliubov. Essa aproximação para mistura é uma generalização natural da mesma aproximação em condensados com uma componente.

Guiados pela referência [70] que estuda o mesmo sistema na aproximação de Bogoliubov e para temperatura nula, reduzimos as equações de Gross-Pitaevskii a uma equação para a fração relativa de átomos dos dois condensados considerando a simetria de calibre do sistema. Essa equação tem a mesma forma da equação para a fração relativa em $T=0$, a diferença entre elas reside no fato dos parâmetros de controle dependerem da temperatura. Naturalmente, se desprezarmos efeitos relacionados com a depleção dos condensados, recuperamos a equação deduzida em [70].

A equação para a fração relativa de partículas nos dois condensados e as equações de Hartree-Fock-Bogoliubov-Popov para as excitações coletivas são acopladas e foram resolvidas auto-consistentemente. Com isso, calculamos, como função da temperatura, a densidade de átomos em cada condensado, a lacuna do ramo óptico e extraímos a 
velocidade do som do sistema através do espectro do ramo acústico.

Analisamos três casos que diferem entre si pelos valores dos parâmetros do sistema, as intensidades da interação entre os átomos e a intensidade do termo de acoplamento de Josephson e consideramos a dessintonia nula nos três casos.

No primeiro caso escolhemos os parâmetros do sistema de acordo com os dados experimentais que, acidentalmente, tem o parâmetro de interação mútua, $A_{e f}$, nulo. Nesse caso existe apenas um estado de equilíbrio como pode ser verificado pela localização da curva descrita pelo sistema no espaço de parâmetros de controle, pois essa curva está sempre na região de apenas uma solução da equação de Gross-Pitaevskii. Vimos que, quando a temperatura tende à temperatura de transição $T_{t} / T_{0}=0.80$, as densidades de átomos nos dois condensados se anulam simultaneamente como conseqüência da coerência da mistura. A velocidade do som decresce monotonicamente como função da temperatura, se anulando na temperatura de transição. A lacuna tem um comportamento análogo mas tem um valor finito na temperatura de transição. Tanto os resultados da velocidade do som quanto da lacuna estão de acordo com os cálculos usando as expressões explícitas para essas grandezas físicas determinadas no capítulo 2.

No segundo caso, escolhemos os parâmetros do sistema de tal modo que, em temperatura nula, a mistura esteja na região onde existe a bi-estabilidade com a dessintonia efetiva positiva. Nesse caso existem dois estados de equilíbrio estáveis, um onde a fração relativa é positiva e outra onde é negativa. Ao aquecermos o sistema a solução com fração positiva aproxima-se da sua correspondente curva de bifurcação e desaparece quando $T_{c} / T_{0} \approx 0.29$. Atribuímos esse desaparecimento a instabilidade do espectro do ramo acústico de excitação. Chegamos a essa conclusão analisando a expressão explícita do quadrado da velocidade do som, grandeza essa extraída do espectro do ramo acústico, e o comportamento dele nas proximidades da curva de bifurcação. Como o quadrado da velocidade do som do sistema decresce abruptamente quando a temperatura se aproxima da temperatura de colapso e como ele é inversamente proporcional a lacuna que é nula quando os parâmetros de controle estão na curva de bifurcação, vemos que o quadrado da velocidade do som diverge negativamente. Um valor negativo do quadrado da velocidade do som implica em valores imaginários para energia do ramo acústico, ou seja, o sistema fica instável antes de atingir a curva de bifurcação provocando o desaparecimento da solução para uma temperatura menor que a temperatura de colapso. 
Por outro lado, se seguirmos a solução com fração negativa, vemos que a curva descrita no espaço dos parâmetros de controle está numa região onde essa solução sempre existe e, em especial, é o único estado de equilíbrio para $T / T_{0} \geq 0.29$. As densidades dos condensados, a lacuna e a velocidade do som comportam-se como no caso anterior com uma temperatura de transição sendo igual à $T_{t} / T_{0}=0.69$.

Resumindo, vemos que, ao aquecer o sistema seguindo a solução com fração positiva, existe uma temperatura próxima a temperatura de colapso para a qual a mistura fica instável podendo, então, colapsar ou decair para o estado de equilíbrio com fração negativa ocorrendo uma variação abrupta da população dos condensados. Assim, próximo a temperatura de colapso o valor das densidades dos condensados permanece finito, enquanto a lacuna e a velocidade do som tendem a zero.

No terceiro caso escolhemos os parâmetros do sistema tal que, em $T=0$, a dessintonia efetiva é nula e a mistura esteja na região de bi-estabilidade. Nesse caso, quando aquecemos o sistema, a curva no espaço de parâmetros de controle para as duas soluções af́astam-se das suas respectivas curvas de bifurcação. Inicialmente as frações positivas e negativas mantêm-se relativamente constantes e ao nos aproximarmos da temperatura de degenerescência das soluções, $T_{d} / T_{0}=0.57$, vão a zero repentinamente. A partir dessa temperatura, o sistema tem apenas um estado de equilíbrio com a fração relativa nula, $\bar{f}=0$, até atingir a temperatura de transição de $T_{t} / T_{0}=0.66$.

$\mathrm{O}$ fato das frações, tanto positiva quanto negativa, irem a zero abruptamente, provoca descontinuidades na derivada calculada na temperatura de degenerescência para as densidades dos condensados, para a lacuna e para a velocidade do som.

Nesse ponto, parece-nos apropriado fazer um breve comentário sobre a validade da teoria de Hartree-Fock-Bogoliubov-Popov. Para o sistema confinado em uma armadilha, conjectura-se que a teoria de Hartree-Fock-Bogoliubov-Popov deve ser quantitativamente correta para temperaturas menores que $0.6 T_{t}$ [64]. Extrapolando essa análise para o sistema homogêneo, vemos que, para o segundo caso, a temperatura do colapso da biestabilidade do sistema está na região de validade da teoria, $T_{c} / T_{t}=0.42$. Entretanto, para o terceiro caso, a temperatura de degenerescência está numa região de temperatura em que a teoria supostamente não é válida quantitativamente, $T_{d} / T_{t}=0.83$, e, portanto, devemos esperar que nossa análise desse fenômeno tenha um caráter qualitativo. 
Para finalizar a conclusão da tese, anunciamos os principais resultados do estudo da mistura. Destacamos,

- Bi-estabilidade dependente da temperatura

- Encontramos dois mecanismos para o fim da bi-estabilidade

* o desaparecimento de um dos estados de equilíbrio causada pela instabilidade do ramo acústico nas proximidades da temperatura de colapso

* os estados de equilíbrio coincidem

- Determinamos três temperaturas importantes para a mistura e suas características

- Na temperatura de transição (para todos os casos)

* a temperatura de transição está num intervalo de $0.63<T_{t} / T_{0}<1$

* a população dos condensados se anulam simultaneamente

* a lacuna do espectro é finita

* a velocidade do som se anula

- Na temperatura de colapso (para o caso em que desaparece uma das soluções)

* a lacuna tende a zero

* a velocidade do som torna-se imaginária e o espectro acústico torna-se instável nas proximidades da temperatura de colapso

- Na temperatura de degenerescência das soluções (para o caso em que nenhuma das soluções cruza com a correspondente curva de bifurcação)

* a população de ambos condensados são iguais, e mantem-se assim até atingir a temperatura de transição

* a lacuna e a velocidade do som apresentam uma descontinuidade na sua derivada 


\section{Apêndice A}

\section{O teorema de Wick em mecânica}

\section{estatística}

Vamos assumir que o operador estatístico $D$ possa ser escrito na seguinte forma:

$$
D=\frac{e^{-\beta K_{e f}}}{\operatorname{Tr} e^{-\beta K_{e f}}}
$$

onde $K_{\text {ef }}$ é um hamiltoniano quadrático e, diferentemente da referência [76], dado por:

$$
K_{e f}=\frac{1}{2} \sum_{i j}\left[h_{i j}\left(\alpha_{i}^{\dagger} \alpha_{j}+\epsilon \alpha_{j} \alpha_{i}^{\dagger}\right)+\Delta_{i j} \alpha_{i}^{\dagger} \alpha_{j}^{\dagger}+\Delta_{i j}^{*} \alpha_{i} \alpha_{j}\right]
$$

como sugerido em [71] para a aproximação de Hartree-Fock-Bogoliubov. O fator $\epsilon$ é igual a +1 para quando $\alpha$ for um operador de bósons e -1 quando for de férmions e, conseqüentemente, os operadores $\alpha_{i}$ e $\alpha_{i}^{\dagger}$ obedecem as regras de comutação e anticomutação canônicas,

$$
\left\{\begin{array}{l}
{\left[\alpha_{i}, \alpha_{j}^{\dagger}\right]_{\epsilon}=\alpha_{i} \alpha_{j}^{\dagger}-\epsilon \alpha_{j}^{\dagger} \alpha_{i}=\delta_{i j}} \\
{\left[\alpha_{i}, \alpha_{j}\right]_{\epsilon}=\alpha_{i} \alpha_{j}-\epsilon \alpha_{j} \alpha_{i}=0}
\end{array}\right.
$$

Como $K_{\text {ef }}$ está na forma quadrática, sempre existe uma transformação nos operadores de criação e aniquilação, $\alpha_{i}^{\dagger}, \alpha_{i}$ que torne o hamiltoniano (A.2) diagonal e que os novos operadores, $\eta_{i}^{\dagger}$ e $\eta_{i}$ obedeçam as mesmas regras de comutação dos antigos.

Considerando essa transformação de Bogoliubov

$$
\left\{\begin{array}{l}
\alpha_{i}^{\dagger}=\sum_{j} u_{i j}^{*} \eta_{j}^{\dagger}+v_{i j} \eta_{j} \\
\alpha_{i}=\sum_{j} u_{i j} \eta_{j}+v_{i j}^{*} \eta_{j}^{\dagger}
\end{array}\right.
$$


vemos que o hamiltoniano assume a forma diagonal, $K_{e f}=\sum_{i} E_{i} \eta_{i}^{\dagger} \eta_{i}$ impondo que os parâmetros da transformação de Bogoliubov satisfaçam a equação de Hartree-FockBogoliubov.

Usando a definição (A.1), demonstramos o teorema de Wick através da média térmica, $\operatorname{Tr}\{a b c \cdots e f D\}$, onde $a b c \cdots e f$ é um produto de um número par de operadores de criação ou de aniquilação. Observamos que o produto de um número ímpar de operadores tem sua média térmica nula pois tal produto não conserva número total de quasi-partículas.

O procedimento para a demonstração foi assumir o operador $a$, indicado na média, como sendo $\alpha_{i}^{\dagger}$ e escrever o traço em termos dos operadores transformados, $\eta_{i}^{\dagger}$ e $\eta_{i}$,

$$
\operatorname{Tr}\left\{\alpha_{i}^{\dagger} b c \cdots e f D\right\}=\sum_{j} u_{i j}^{*} \operatorname{Tr}\left\{\eta_{j}^{\dagger} b c \cdots e f D\right\}+v_{i j} \operatorname{Tr}\left\{\eta_{j} b c \cdots e f D\right\}
$$

Ao comutar sucessivamente $\eta_{j}^{\dagger}$ com os operadores $b c \cdots e f D$, pudemos escrever a média como

$$
\begin{aligned}
& \operatorname{Tr}\left\{\eta_{j}^{\dagger} b c d \cdots e f D\right\}= \\
= & {\left[\eta_{j}^{\dagger}, b\right] \operatorname{Tr}\{c d \cdots e f D\}+\epsilon\left[\eta_{j}^{\dagger}, c\right] \operatorname{Tr}\{b d \cdots e f D\}+} \\
+ & {\left[\eta_{j}^{\dagger}, d\right] \operatorname{Tr}\{b c \cdots e f D\}+\cdots+\left[\eta_{j}^{\dagger}, f\right] \operatorname{Tr}\{b c d \cdots e D\}+} \\
+ & \epsilon \operatorname{Tr}\left\{b c \cdots e f \eta_{j}^{\dagger} D\right\} .
\end{aligned}
$$

Devido o hamiltoniano, $K_{e f}$, ser diagonal nesses novos operadores, vemos que

$$
\operatorname{Tr}\left\{b c \cdots e f \eta_{j}^{\dagger} D\right\}=e^{\beta E_{j}} \operatorname{Tr}\left\{\eta_{j}^{\dagger} b c d \cdots e f D\right\}
$$

pois $\eta_{j}^{\dagger} D=e^{\beta E_{j}} D \eta_{j}^{\dagger}$. Assim temos

$$
\begin{aligned}
& \operatorname{Tr}\left\{\eta_{j}^{\dagger} b c d \cdots e f D\right\}= \\
= & \frac{\left[\eta_{j}^{\dagger}, b\right]}{\left(1-\epsilon e^{\beta E_{j}}\right)} \operatorname{Tr}\{c d \cdots e f D\}+\epsilon \frac{\left[\eta_{j}^{\dagger}, c\right]}{\left(1-\epsilon e^{\beta E_{j}}\right)} \operatorname{Tr}\{b d \cdots e f D\}+ \\
+ & \frac{\left[\eta_{j}^{\dagger}, d\right]}{\left(1-\epsilon e^{\beta E_{j}}\right)} \operatorname{Tr}\{b c \cdots e f D\}+\cdots+\frac{\left[\eta_{j}^{\dagger}, f\right]}{\left(1-\epsilon e^{\beta E_{j}}\right)} \operatorname{Tr}\{b c d \cdots e D\} .
\end{aligned}
$$

Da mesma maneira, e lembrando que $\eta_{j} D=e^{-\beta E_{j}} D \eta_{j}$, temos

$$
\begin{aligned}
& \operatorname{Tr}\left\{\eta_{j} b c d \cdots e f D\right\}= \\
= & \frac{\left[\eta_{j}, b\right]}{\left(1-\epsilon e^{\left.-\beta E_{j}\right)}\right.} \operatorname{Tr}\{c d \cdots e f D\}+\epsilon \frac{\left[\eta_{j}, c\right]}{\left(1-\epsilon e^{-\beta E_{j}}\right)} \operatorname{Tr}\{b d \cdots e f D\}+ \\
+ & \frac{\left[\eta_{j}, d\right]}{\left(1-\epsilon e^{-\beta E_{j}}\right)} \operatorname{Tr}\{b c \cdots e f D\}+\cdots+\frac{\left[\eta_{j}, f\right]}{\left(1-\epsilon e^{-\beta E_{j}}\right)} \operatorname{Tr}\{b c d \cdots e D\} .
\end{aligned}
$$


Usando (A.8) e (A.9) em (A.5) e agrupando de forma que fique em evidência os traços, temos

$$
\begin{aligned}
& \operatorname{Tr}\left\{\alpha_{i}^{\dagger} b c \cdots e f D\right\}= \\
= & \sum_{j}\left(\frac{u_{i j}^{*}\left[\eta_{j}^{\dagger}, b\right]}{\left(1-\epsilon e^{\beta E_{j}}\right)}+\frac{v_{i j}\left[\eta_{j}, b\right]}{\left(1-\epsilon e^{-\beta E_{j}}\right)}\right) \operatorname{Tr}\{c d \cdots e f D\}+ \\
+ & \epsilon \sum_{j}\left(\frac{u_{i j}^{*}\left[\eta_{j}^{\dagger}, c\right]}{\left(1-\epsilon e^{\beta E_{j}}\right)}+\frac{v_{i j}\left[\eta_{j}, c\right]}{\left(1-\epsilon e^{-\beta E_{j}}\right)}\right) \operatorname{Tr}\{b d \cdots e f D\}+\cdots \\
+ & \sum_{j}\left(\frac{u_{i j}^{*}\left[\eta_{j}^{\dagger}, f\right]}{\left(1-\epsilon e^{\beta E_{j}}\right)}+\frac{v_{i j}\left[\eta_{j}, f\right]}{\left(1-\epsilon e^{-\beta E_{j}}\right)}\right) \operatorname{Tr}\{b c d \cdots e D\}
\end{aligned}
$$

e do mesmo modo vemos que para $a=\alpha_{i}$

$$
\begin{aligned}
& \operatorname{Tr}\left\{\alpha_{i} b c \cdots e f D\right\}= \\
= & \sum_{j}\left(\frac{\left[\eta_{j}, b\right] u_{i j}}{\left(1-\epsilon e^{-\beta E_{j}}\right)}+\frac{\left[\eta_{j}^{\dagger}, b\right] v_{i j}^{*}}{\left(1-\epsilon e^{\beta E_{j}}\right)}\right) \operatorname{Tr}\{c d \cdots e f D\}+ \\
+ & \epsilon \sum_{j}\left(\frac{\left[\eta_{j}, c\right] u_{i j}}{\left(1-\epsilon e^{-\beta E_{j}}\right)}+\frac{\left[\eta_{j}^{\dagger}, c\right] v_{i j}^{*}}{\left(1-\epsilon e^{\beta E_{j}}\right)}\right) \operatorname{Tr}\{b d \cdots e f D\}+\cdots \\
+ & \sum_{j}\left(\frac{\left[\eta_{j}, f\right] u_{i j}}{\left(1-\epsilon e^{\left.-\beta E_{j}\right)}\right.}+\frac{\left[\eta_{j}^{\dagger}, f\right] v_{i j}^{*}}{\left(1-\epsilon e^{\beta E_{j}}\right)}\right) \operatorname{Tr}\{b c d \cdots e D\} .
\end{aligned}
$$

Usando o mesmo procedimento acima, podemos mostrar que a média térmica do produto de dois operadores $a$ e $b$ é dada por:

$$
\langle a b\rangle=\left\{\begin{array}{lll}
\sum_{j}\left(\frac{u_{i j}^{*}\left[\eta_{j}^{\dagger}, b\right]}{\left(1-\epsilon e^{\beta E_{j}}\right)}+\frac{v_{i j}\left[\eta_{j}, b\right]}{\left(1-\epsilon e^{-\beta E_{j}}\right)}\right) & \text { se } & a=\alpha_{i}^{\dagger} \\
\sum_{j}\left(\frac{\left[\eta_{j}, b\right] u_{i j}}{\left(1-\epsilon e^{-\beta E_{j}}\right)}+\frac{\left[\eta_{j}^{\dagger}, b\right] v_{i j}^{*}}{\left(1-\epsilon e^{\beta E_{j}}\right)}\right) & \text { se } & a=\alpha_{i}
\end{array} .\right.
$$

Assim, podemos escrever a média térmica como

$$
\begin{aligned}
\operatorname{Tr}\{a b c \cdots e f D\} & =\langle a b\rangle \operatorname{Tr}\{c d \cdots e f D\}+\epsilon\langle a c\rangle \operatorname{Tr}\{b d \cdots e f D\}+ \\
& +\langle a d\rangle \operatorname{Tr}\{b c \cdots e f D\}+\cdots+\langle a f\rangle \operatorname{Tr}\{b c d \cdots e D\}
\end{aligned}
$$

e, portanto, demonstrar o teorema de Wick em mecânica estatísticas na qual a regra de contração é dada em (A.12).

Como exemplo de uma aplicação do teorema de Wick, calculamos a média térmica do produto de quatro operadores bosônicos, $c_{\alpha(\vec{k}+\vec{q})}^{\dagger}, c_{\beta\left(\overrightarrow{k^{\prime}}-\vec{q}\right)}^{\dagger}, c_{\alpha \vec{k}}$ e $c_{\beta \vec{k}^{\prime}}$. Usando (A.13) temos

$$
\begin{aligned}
& \operatorname{Tr}\left\{D c_{\alpha(\vec{k}+\vec{q})}^{\dagger} c_{\beta\left(\vec{k}^{\prime}-\vec{q}\right)}^{\dagger} c_{\alpha \vec{k}} c_{\beta \vec{k}^{\prime}}\right\}=\left\langle c_{\alpha(\vec{k}+\vec{q})}^{\dagger} c_{\beta\left(\overrightarrow{k^{\prime}}-\vec{q}\right)}^{\dagger}\right\rangle \operatorname{Tr}\left\{D c_{\alpha \vec{k}} c_{\beta \vec{k}^{\prime}}\right\}+ \\
+ & \left\langle c_{\alpha(\vec{k}+\vec{q})}^{\dagger} c_{\alpha \vec{k}}\right\rangle \operatorname{Tr}\left\{D c_{\beta\left(\vec{k}^{\prime}-\vec{q}\right)}^{\dagger} c_{\beta \vec{k}^{\prime}}\right\}+\left\langle c_{\alpha(\vec{k}+\vec{q})}^{\dagger} c_{\beta \vec{k}^{\prime}}\right\rangle \operatorname{Tr}\left\{D c_{\beta\left(\overrightarrow{k^{\prime}}-\vec{q}\right)}^{\dagger} c_{\alpha \vec{k}}\right\}
\end{aligned}
$$


e com isso

$$
\begin{aligned}
& \operatorname{Tr}\left\{D c_{\alpha(\vec{k}+\vec{q})}^{\dagger} c_{\beta\left(\vec{k}^{\prime}-\vec{q}\right)}^{\dagger} c_{\alpha \vec{k}} c_{\beta \vec{k}^{\prime}}\right\}=\left\langle c_{\alpha(\vec{k}+\vec{q})}^{\dagger} c_{\beta\left(\vec{k}^{\prime}-\vec{q}\right)}^{\dagger}\right\rangle\left\langle c_{\alpha \vec{k}} c_{\beta \vec{k}^{\prime}}\right\rangle+ \\
+ & \left\langle c_{\alpha(\vec{k}+\vec{q})}^{\dagger} c_{\alpha \vec{k}}\right\rangle\left\langle c_{\beta\left(\vec{k}^{\prime}-\vec{q}\right)}^{\dagger} c_{\beta \vec{k}^{\prime}}\right\rangle+\left\langle c_{\alpha(\vec{k}+\vec{q})}^{\dagger} c_{\beta \vec{k}^{\prime}}\right\rangle\left\langle c_{\beta\left(\overrightarrow{k^{\prime}}-\vec{q}\right)}^{\dagger} c_{\alpha \vec{k}}\right\rangle .
\end{aligned}
$$

onde as contrações de (A.15) são calculadas através de (A.12) e dadas por

$$
\left\langle c_{\alpha \vec{k}} c_{\beta \vec{k}^{\prime}}\right\rangle=\sum_{\gamma}\left[\left(\frac{1}{2}+\nu_{\gamma}(\vec{k})\right)\left(u_{\alpha \gamma}(\vec{k}) v_{\beta \gamma}^{*}(\vec{k})+v_{\alpha \gamma}^{*}(\vec{k}) u_{\beta \gamma}(\vec{k})\right)\right] \delta_{\vec{k},-\vec{k}^{\prime}}
$$

e

$$
\left\langle c_{\alpha \vec{k}}^{\dagger} c_{\beta \vec{k}^{\prime}}\right\rangle=\sum_{\gamma}\left[\nu_{\gamma}(\vec{k}) u_{\alpha \gamma}(\vec{k}) u_{\beta \gamma}^{*}(\vec{k})+\left(1+\nu_{\gamma}(\vec{k})\right) v_{\alpha \gamma}(\vec{k}) v_{\beta \gamma}^{*}(\vec{k})\right] \delta_{\vec{k}, \vec{k}^{\prime}}
$$

$$
\operatorname{com} \nu_{\gamma}(\vec{k})=\frac{1}{e^{\beta E_{\gamma}(\vec{k})}-1} \text {. }
$$




\section{Apêndice B}

\section{Determinação das temperaturas de transição}

\section{B.1 Para um sistema ideal de uma componente}

Para um sistema ideal e homogêneo de bósons, vemos que o hamiltoniano leva só em conta a energia cinética e, portanto, é dado por

$$
H=\sum_{\vec{k}} \frac{\hbar^{2} k^{2}}{2 m} a_{\vec{k}}^{\dagger} a_{\vec{k}} .
$$

Com o hamiltoniano diagonal no momento é direto ver que o número de ocupação é dado por

$$
\nu_{\vec{k}}=\frac{1}{e^{\beta\left(\epsilon_{k}-\mu\right)}-1} .
$$

A partir do número de ocupação calculamos o número de partículas médio do sistema,

$$
N=\sum_{\vec{k}} \nu_{\vec{k}}
$$

e a energia média do sistema,

$$
E=\sum_{\vec{k}} \epsilon_{k} \nu_{\vec{k}}
$$

Ao tomar o limite termodinâmico o espectro de excitação se torna contínuo, e disso temos

$$
\sum_{\vec{k}} \rightarrow \frac{\Omega}{(2 \pi)^{3}} \int d^{3} \vec{k}
$$


Fazendo a mudança de variável de momento para energia cinética do sistema em (B.3) reescrevemos o número médio de partículas no sistema em função de uma integral dada por

$$
\rho=\frac{1}{4 \pi^{2}}\left(\frac{2 m}{\hbar^{2}}\right)^{\frac{3}{2}} \int_{0}^{\infty} \frac{\epsilon^{\frac{1}{2}}}{e^{\beta(\epsilon-\mu)}-1} d \epsilon
$$

Fazendo mais uma mudança de variável apropriada na integral tal que $x=\beta \epsilon \mathrm{e} z=e^{\beta \mu}$ chegamos à

$$
\rho=\frac{1}{\lambda_{T}^{3}} g_{3 / 2}(z)
$$

onde $g_{3 / 2}(z) \equiv \sum_{l=1}^{\infty} \frac{z^{l}}{l^{3 / 2}}$ e $\lambda_{T}$ corresponde ao comprimento de onda térmico ${ }^{1}$ dado por

$$
\lambda_{T}=\sqrt{\frac{2 \pi \hbar^{2}}{m k_{B} T}} .
$$

Notamos que a equação (B.6) tem sentido somente para $\epsilon-\mu \geq 0$, caso contrário o número de ocupação, $\nu_{\epsilon}$, será menor que zero para algum valor de $\epsilon$. Como $\epsilon \geq 0$, vemos que para satisfazer a condição de $\nu_{\epsilon}>0$ temos que garantir a negatividade do potencial químico, $\mu \leq 0$. Com isso, definimos uma temperatura de transição, $T=T_{0}$, em que o sistema tenha um potencial químico nulo, de (B.7) temos

$$
\rho \lambda_{0}^{3}=g_{3 / 2}(1) \approx 2.612,
$$

onde $\lambda_{0}$ é o comprimento de onda térmico para essa temperatura. Vemos que essa temperatura de transição (em que o potencial químico é nulo) é comparável a raiz cúbica do volume específico do gás,

$$
T_{0}=\frac{2 \pi \hbar^{2}}{m k_{B}}\left(\frac{\rho}{g_{3 / 2}(1)}\right)^{\frac{2}{3}} .
$$

Quando a temperatura está abaixo da temperatura de transição, $T_{0}$, há a formação do condensado [73].

\section{B.2 Para um sistema ideal de duas componentes com acoplamento Josephson}

Supondo que o sistema contenha duas espécies de partículas com acoplamento Josephson e sabendo que as partículas não interagem entre si, a dinâmica do sistema é dado pelo

\footnotetext{
${ }^{1}$ Supomos que a energia cinética corresponde a térmica por $\frac{\hbar^{2} k^{2}}{2 m}=k_{B} T$ sabendo que $k=2 \pi / \lambda_{T}$
} 
hamiltoniano

$$
H=\sum_{\alpha} \sum_{\vec{k}} \frac{\hbar^{2} k^{2}}{2 m} a_{\alpha \vec{k}}^{\dagger} a_{\alpha \vec{k}}+\sum_{\alpha \beta} \sum_{\vec{k}} J_{\alpha \beta} a_{\alpha \vec{k}}^{\dagger} a_{\beta \vec{k}}
$$

que leva em conta a energia cinética e o termo de Josephson que acopla os estados hiperfinos.

Diagonalizamos esse hamiltoniano por uma transformação canônica para novos operadores de bósons dado por

$$
\left\{\begin{array}{l}
b_{+\vec{k}}=\frac{1}{\sqrt{2}}\left(a_{1 \vec{k}}+a_{2 \vec{k}}\right) \\
b_{-\vec{k}}=\frac{1}{\sqrt{2}}\left(a_{1 \vec{k}}-a_{2 \vec{k}}\right)
\end{array} .\right.
$$

Dessa transformação vemos que as energias de excitação do sistema livre com acoplamento Josephson são

$$
E_{ \pm}(\vec{k})=\frac{\hbar^{2} k^{2}}{2 m} \pm J
$$

Dessa forma, vemos que o número de ocupação é dado por

$$
\nu_{ \pm \vec{k}}=\frac{1}{e^{\beta\left(E_{ \pm}(\vec{k})-\mu\right)}-1} .
$$

E, com isso, calculamos o número de partículas médio do sistema,

$$
N=\sum_{ \pm} \sum_{\vec{k}} \nu_{ \pm \vec{k}}
$$

Ao tomar o limite termodinâmico temos para o número médio de partículas

$$
\rho=\sum_{ \pm} \frac{1}{(2 \pi)^{3}} \int \nu_{ \pm \vec{k}} d^{3} k
$$

e, fazendo a mudança de variável de momento para energia cinética, vemos que o número de partícula é dado por

$$
\rho=\sum_{ \pm} \frac{1}{4 \pi^{2}}\left(\frac{2 m}{\hbar^{2}}\right)^{\frac{3}{2}} \int_{0}^{\infty} \frac{\epsilon^{\frac{1}{2}}}{e^{\beta(\epsilon \pm J-\mu)}-1} d \epsilon .
$$

Mais uma vez, notamos que a equação (B.17) tem sentido somente para $\epsilon-J-\mu \geq 0$. Como $\epsilon \geq 0$, vemos que para satisfazer a condição de $\nu_{\epsilon}>0$ temos que garantir que o potencial químico seja menor que o valor negativo do coeficiente de Josephson, $\mu \leq-J$. 
Com isso, lembrando que na temperatura de transição, $T=T_{t}$ o sistema tem um potencial químico igual à $-J$, temos de (B.7)

$$
\rho=\frac{1}{\lambda_{t}^{3}}\left(g_{3 / 2}(1)+g_{3 / 2}\left(e^{-2 J \beta_{t}}\right)\right) .
$$

o que faz chegarmos a uma equação transcendental para a temperatura de transição,

$$
k_{B} T_{t}=\frac{2 \pi \hbar^{2}}{m} \rho^{2 / 3} \frac{1}{\left(g_{3 / 2}(1)+g_{3 / 2}\left(e^{-2 J \beta_{t}}\right)\right)^{2 / 3}} .
$$

que pode ser escrita como

$$
\frac{T_{t}}{T_{0}}=\left(\frac{g_{3 / 2}(1)}{\left(g_{3 / 2}(1)+g_{3 / 2}\left(e^{-2 J \beta_{t}}\right)\right)}\right)^{2 / 3} .
$$

\section{B.3 Para um sistema interagente de duas componen- tes com acoplamento Josephson}

Sabemos que além da temperatura de transição do sistema não há um agrupamento macroscópico de partículas no mesmo estado quântico. Conseqüentemente, as densidades de partículas condensadas em cada estado hiperfino são nulas nessa temperatura. Dessa forma vemos que, nas equações de Hartree-Fock-Bogoliubov na aproximação de Popov, os termos que acoplam os coeficientes da transformação de Bogoliubov vão a zero e o hamiltoniano efetivo do sistema se reduz a

$$
\begin{aligned}
K_{e f} & =\sum_{\vec{k}}\left(\frac{\hbar^{2} k^{2}}{2 m}+\sqrt{\frac{1-\bar{f}}{1+\bar{f}}}\left(J+\lambda_{12} d_{12}\right)\right) c_{1 \vec{k}}^{\dagger} c_{1 \vec{k}}+ \\
& +\sum_{\vec{k}}\left(\frac{\hbar^{2} k^{2}}{2 m}+\sqrt{\frac{1+\bar{f}}{1-\bar{f}}}\left(J+\lambda_{12} d_{12}\right)\right) c_{2 \vec{k}}^{\dagger} c_{2 \vec{k}}+ \\
& +\sum_{\vec{k}}\left(J+\lambda_{12} d_{12}\right) c_{1 \vec{k}}^{\dagger} c_{2 \vec{k}}+ \\
& +\sum_{\vec{k}}\left(J+\lambda_{12} d_{12}\right) c_{2 \vec{k}}^{\dagger} c_{1 \vec{k}} .
\end{aligned}
$$

onde assumimos a diferença de fase entre os condensados como sendo $\pi$. Diagonalizamos (B.21) pela transformação (B.12) e obtemos como energias de excitação

$$
\left\{\begin{array}{l}
E_{+}(\vec{k})=\frac{\hbar^{2} k^{2}}{2 m}+\frac{2 J_{e f}}{\sqrt{1-\bar{f}^{2}}} \\
E_{-}(\vec{k})=\frac{\hbar^{2} k^{2}}{2 m}
\end{array}\right.
$$


Com o espectro de excitação do sistema calculamos o número de ocupação de estados e, conseqüentemente, a densidade do sistema da mesma forma que feita na seç̧ão anterior. Vemos, com os mesmos argumentos usados para o caso ideal, que a temperatura de transição do sistema é dada pela solução da equação transcendental

$$
\frac{T_{t}}{T_{0}}=\left(\frac{g_{3 / 2}(1)}{\left(g_{3 / 2}(1)+g_{3 / 2}\left(e^{-2 \bar{J} \beta_{t}}\right)\right)}\right)^{2 / 3}
$$

onde $\tilde{J}=\frac{J_{e f}}{\sqrt{1-\bar{f}^{2}}}$. Essa equação pode ser reescrita como

$$
\frac{T_{t}}{T_{0}}=\left(\frac{g_{3 / 2}(1)}{\left(g_{3 / 2}(1)+g_{3 / 2}\left(e^{-X \frac{T_{0}}{T_{t}}}\right)\right)}\right)^{2 / 3}
$$

onde $X=\frac{2 \tilde{J}}{k_{B} T_{0}}$ é o parâmetro usado para descrever o gráfico da figura 3.5 . 


\section{Bibliografia}

[1] Anderson M H, Ensher J R, Mathews M R, Wieman C E and Cornell E A 1995 Science 269198

[2] Davis K B, Mewes M O, Andrews M R, van Druten N J, Durfee D D, Kurn D M and Ketterle W 1995 Phys. Rev. Lett. 753969

[3] Bradley C C, Sackett C A, Tollett J J and Hulet R G 1995 Phys. Rev. Lett. 751687

[4] Cornell E A and Wieman C E 2002 Rev. Mod. Phys. 74875

[5] Ketterle W 2002 Rev. Mod. Phys. 741131

[6] Bose S N 1924 Z. Phys. 26178

[7] Einstein A 1925 Sitzungsberichte der Preussischen Akademie der Wisseschaften zu Berlin,rept. 3, p. 18

[8] London F 1938 Phys. Rev. 54947

[9] Tisza L 1938 Nature 141273

[10] Landau L D 1941 J. Phys. USSR 571

[11] Landau L D 1947 J. Phys. USSR 1191

[12] Landau L D 1949 Phys. Rev. 75884

[13] Leggett A J 1999 Rev. Mod. Phys. 71 S318

[14] Bogoliubov N N 1947 J. Phys. USSR 1123

[15] Feynman R P 1954 Phys. Rev. 911301 
[16] Feynman R P and Cohen M 1956 Phys. Rev. 1021184

[17] Phillips W D 1998 Rev. Mod. Phys. 70721

[18] Leggett A 2001 Rev. Mod. Phys. 73307

[19] Jin D S, Ensher J R, Matthews M R, Wieman C E and Cornell E A 1996 Phys. Rev. Lett. 77420

[20] Mewes M -O, Andrews M R, van Drutten N J, Kurn D M, Durfee D S, Townsend C G and Ketterle W 1996 Phys. Rev. Lett. 77988

[21] Stenger J, Inouye S, Chikkatur A P, Stamper-Kurn D M, Pritchard D E and Ketterle W 1999 Phys. Rev. Lett. 824569

[22] Stamper-Kurn D M, Chikkatur A P, Gorlitz A, Inouye S, Gupta S, Pritchard D E and Ketterle W 1999 Phys. Rev. Lett. 832876

[23] Steinhauer J, Ozeri R and Davidson N 2002 Phys. Rev. Lett. 88120407

[24] Vogels J M, Xu K, Raman C, Abo-Shaeer J R and Ketterle W 2002 Phys. Rev. Lett. 88060402

[25] Myatt C J, Burt E A, Ghrit R W, Cornell E A and Wieman C E 1997 Phys. Rev. Lett. 78586

[26] Stamper-Kurn D M, Andrews M R, Chikkatur A P, Inouye S, Miesner H -J, Stenger J and Ketterle W 1998 Phys. Rev. Lett. 802027

[27] Stenger J, Inouye S, Stamper-Kurn D M, Miesner H-J, Chikkatur A P and Ketterle W 1998 Nature 396345

[28] Andrews M R, Townsend C G, Miesner H-J, Durfee D S, Kurn D M and Ketterle W 1997Science 275637

[29] Röhrl A, Naraschewski M, Schenzle A, and Wallis H 1997 Phys. Rev. Lett. 784143

[30] Matthews M R, Hall D S, Jin D S, Ensher J R, Wieman C E, Cornell E A, Dalfovo F, Minniti C and Stringari S 1998 Phys. Rev. Lett. 81243 
[31] Hall D S, Matthews M R, Ensher J R, Wieman C E and Cornell E A 1998 Phys. Rev. Lett. 81, 1539

[32] Matthews M R, Anderson B P, Haljan P C, Hall D S, Holland M J, Williams J E, Wieman C E and Cornell E A 1999 Phys. Rev. Lett. 833358

[33] Modugno G, Ferrari G, Roati G, Brecha R J, Simoni A and Inguscio M 2001 Science 2941320

[34] Schreck F, Khaykovich L, Corwin K L, Ferrari G, Bourdel T, Cubizolles J and Salomon C 2001 Phys. Rev. Lett. 87080403

[35] Hadzibabic Z, Stan C A, Gupta S, Zwierlein M W, Görlitz A and Ketterle W 2002 Phys. Rev. Lett. 88160401

[36] Jin D S, Matthews M R, Ensher J R, Wieman C E and Cornell E A 1997 Phys. Rev. Lett. 78764

[37] Stamper-Kurn D M, Miesner H -J, Inouye S, Andrews M R and Ketterle W 1998 Phys. Rev. Lett. 81500

[38] Gross E P 1958 Ann. Phys. 457

[39] Gross E P 1960 Ann. Phy. 9292

[40] Pitaevskii L P 1961 Sov. Phys. (JETP) 13451

[41] Edwards M and Burnett K 1995 Phys. Rev. A 511382

[42] Edwards M, Dodd R J, Clark C W, Rupretch P A and Burnett K 1996 Phys. Rev. A 53 R1950

[43] Baym G and Pethick C J 1996 Phys. Rev. Lett. 766

[44] Ruprecht P A, Holland M J, Burnett K and Edwards M 1995 Phys. Rev. A 514704

[45] Stringari S 1996 Phys. Rev. Lett. 772360

[46] Singh K G and Rokhsar D S 1996 Phys. Rev. Lett. 771667 
[47] Edwards M, Ruprecht P A, Burnett K, Dodd R J and Clark 1996 Phys. Rev. Lett. 771671

[48] Pérez-García V M, Michinel H, Cirac J I, Lewenstein M, and Zoller P 1997 Phys. Rev. A 561424

[49] Pires M O da C and de Passos E J V 2000 J. Phys. B: At. Mol. Opt. Phys. 333929

[50] Courteille Ph W, Bagnato V S and Yukalov V I 2001 Laser Physics 11659

[51] Williams J, Walser R, Cooper J, Cornell E and Holland M 2000 Phys. Rev. A 61 033612

[52] Ho T-L and Shenoy V B 1996 Phys. Rev. Lett. 773276

[53] Busch Th, Cirac J I, Pérez-García V M and Zoller P 1997 Phys. Rev. A 562978

[54] Pu H and Bigelow N P 1998 Phys. Rev. Lett. 801134

[55] Graham R and Walls D 1998 Phys. Rev. A 57484

[56] Gordon D and Savage C M 1998 Phys. Rev. A 581440

[57] Öhberg P 1999 Phys. Rev. A 59634

[58] Search C P, Rojo A G and Berman P R 2001 Phys. Rev. A 64013615

[59] Griffin A 1996 Phys. Rev. B 539341

[60] Hugenholtz N M and Pines D 1959 Phys. Rev. 116489

[61] Tommasini P, de Passos E J V, Pires M O C and de Toledo Piza A F R $2005 \mathrm{~J}$. Phys.: Condens. Matter 173165

[62] Popov A 1987 Functional Integrals and Collective Excitations (Cambrigde University Press, Cambrigde)

[63] Hutchinson D A W, Zaremba E and Griffin A 1997 Phys. Rev. Lett. 781842

[64] Dodd R J, Edwards M, Clark C W and Burnett K 1998 Phys. Rev. A 57 R32

[65] Minguzzi A and Tosi M P 1997 Journal of Physics: Condensed Matter 910211 
[66] Xia-Ji Liu, Hui Hu, Minguzzi A and Tosi M P 2004 Phys. Rev. A 69043605

[67] Öhberg P 2000 Phys. Rev. A 61013601

[68] Bassichis W H 1964 Phys. Rev. 134 A543

[69] Goldstein E V and Meystre P 1997 Phys. Rev. A 552935

[70] Tommasini P, de Passos E J V, de Toledo Piza A F R and Hussein M S 2003 Phys. Rev. A 67023606

[71] Blaizot J P and Ripka G 1986 Quantum Theory of Finite Systems (Cambridge, MA:MIT Press)

[72] de Gennes P G 1966 Superconductivity of Metals and Alloys (Benjamin, New York)

[73] Huang K 1963 Statistical Mechanics (Wesley and Sons.)

[74] Lifshitz E M and Pitaevskii L P 1958 Statistical Physics Part 2 (Pergamon Press, Oxford)

[75] Kittel C 1995 Introduction to Solid State Physics (Willey Publishers, New York)

[76] Gaudin M 1960 Nuclear Physics 1589 